\title{
Voronoi polygons and self-consistent technique used to compute the airflow resistivity of randomly placed fibers in glass wool
}

Tarnow, Viggo

Published in:

Acoustical Society of America. Journal

Publication date:

2002

Document Version

Publisher's PDF, also known as Version of record

Link back to DTU Orbit

Citation (APA):

Tarnow, V. (2002). Voronoi polygons and self-consistent technique used to compute the airflow resistivity of randomly placed fibers in glass wool. Acoustical Society of America. Journal, 111(5), 2378-2378.

\section{General rights}

Copyright and moral rights for the publications made accessible in the public portal are retained by the authors and/or other copyright owners and it is a condition of accessing publications that users recognise and abide by the legal requirements associated with these rights.

- Users may download and print one copy of any publication from the public portal for the purpose of private study or research.

- You may not further distribute the material or use it for any profit-making activity or commercial gain

- You may freely distribute the URL identifying the publication in the public portal 


\title{
Session 2aAA
}

\section{Architectural Acoustics: Adaptive Reuse of Vaudeville and Movie Palaces for Modern Music and Theater Performance}

\author{
Carl P. Giegold, Chair \\ Kirkegaard Associates, 801 West Adams Street, Suite 800, Chicago, Illinois 60607 \\ Chair's Introduction-10:30 \\ Invited Papers \\ $10: 35$
}

2aAA1. Acoustical components of the Orpheum Theatre renovation. David A. Conant (McKay Conant Brook, Inc., 5655 Lindero Cyn Rd., Ste. 325, Westlake Village, CA 91362, DConant@ MCBinc.com)

The before and after acoustically-important listening conditions and measurements are described for a $\$ 14.2$ million restoration and renovation of this 1,400-seat grand 1929 Movie Palace-at one time the tallest building in Phoenix. Great care was taken to restore all the acoustically good parts of the original design and to subtly modify the acoustically-troublesome parts (including severely focusing dome and sidewalls) so they looked the same but performed properly. A beautiful playhouse was achieved in 1997 with fine, fixed acoustics and conventional audio reinforcement, a surrounding Italian village, plenty of gilt, moving clouds and sunsets overhead. Today, the venue successfully hosts shows from performance art to ballet to jazz. It was not intended (acoustically) to serve classical music but does so on occasion.

\section{0:55}

2aAA2. A case study: Transforming a vaudeville theatre into a 21st century multi-purpose hall. Richard H. Talaske, Ian B. Hoffman, and Jonathan P. Laney (The Talaske Group, Inc., 105 N. Oak Park Ave., Oak Park, IL, rick@talaske.com)

Originally constructed in 1927 as an atmospheric, vaudeville theatre, the Coronado enjoyed 50 years as host to a true variety of events. In the 1970s, functional and space limitations became a catalyst for touring events to avoid the Coronado, and the theatre fell into disrepair. In the mid-1990s, a renovation was planned (completed January 2001) to restore the glory of the original theatre and upgrade it to be a viable multi-purpose hall for the 21 st century. By annexing two neighboring buildings, many of the space limitations could be overcome in both the front- and back-of-house. The scope included a significantly enlarged stage and stagehouse, performer support spaces, expanded lobby spaces, quiet ventilation systems, and a new music rehearsal room. Acoustically, a primary goal was to upgrade the hall for both reinforced music/theatre performances and unreinforced, orchestral performances. However, historical regulations prevented modification to the room shape and configuration. In order to make the room suitable for unreinforced music, two primary acoustic features were instituted - a semi-custom concert enclosure for the stage and an acoustic enhancement system for the audience chamber. For theatrical and popular music events, a new performance audio system was designed and integrated into the hall.

\section{1:15}

2aAA3. Vaudeville-era renovations from the architect's point of view. Albert L. Filoni (MacLachlan, Cornelius \& Filoni, Inc., 200 The Bank Tower, 307 4th Ave., Pittsburgh, PA 15222, afiloni@mcfarchitects.com)

Interaction between the architect and acoustics consultant on renovation and restoration projects often must reconcile recommendations critical to acoustics goals with uncooperative existing architecture or unwilling landmarks jurisdictions. Through case studies including the renovation of the Pittsburgh Symphony Orchestra's Heinz Hall, the author will discuss the relationship between the architect and acoustics consultant in resolving the design issues inherent in vaudeville-era buildings.

\section{Contributed Papers}

\section{1:35}

2aAA4. The renovation of Madison's Oscar Mayer Theater. Joseph W. A. Myers and Paul T. Calamia (Kirkegaard Assoc., 801 W. Adams St., 8th Fl., Chicago, IL 60607)

Originally opened in 1928 as the Capitol Theatre, the Oscar Mayer Theatre in Madison, WI underwent substantial renovation in 1980 to support its current performance program. The theatre is now home to the Madison Symphony and the Madison Opera, and is used for ballet, touring shows, and popular concerts as well. As part of the ongoing Overture Project which will transform the theatre's home, the Madison Civic Center, into the Overture Center, the Oscar Mayer Theatre will be further improved to support its new role. In this paper we will discuss the reasoning behind the upcoming renovation within the context of the Overture Project, we will describe the pending architectural modifications to the theatre, and we will discuss the intended changes in acoustics. Computer modeling results will also be presented for the existing and renovated conditions. 
2aAA5. There's nothing Majestic about burlesque. Christopher Jaffe and Robin Glosemeyer (Jaffe Holden Acoust., 114A Washington St., Norwalk, CT 06854)

Turn-of-the-century vaudeville and movie palaces initially seem to lend themselves to use as concert halls or multipurpose theatres. Basic features, including existing structure, appropriate location, and desirable seat count, already exist. But, upon further investigation, these palaces stage depth, undersized stage wings, small orchestra pit, deep balcony overhangs, and low ceiling height in stage house and/or audience chamber are common deficiencies which must be addressed in successful conversions. Despite these challenges, converting an existing building is often a viable option for many communities. This paper will investigate several representative palaces which were modified to accommodate modern symphonic and theatrical productions between 1959 and the present.

TUESDAY MORNING, 4 JUNE 2002

LE BATEAU ROOM, 8:00 TO 11:35 A.M.

\title{
Session 2aAB
}

\section{Animal Bioacoustics: Session on Marine Mammal Bioacoustics in Honor of William E. Evans I}

\author{
Jeanette A. Thomas, Cochair \\ Laboratory of Sensory Biology, Western Illinois University, 3561 60th Street, Moline, Illinois 61265 \\ Ann E. Bowles, Cochair \\ Hubbs-Sea World Research Institute, 2595 Ingraham Street, San Diego, California 92109
}

Chair's Introduction-8:00

\section{Invited Papers}

\section{$8: 15$}

2aAB1. Perception time and movement time in dolphin pulsing and whistling. Sam Ridgway and Donald Carder (SPAWAR Systems Ctr. San Diego, Div. D235, 53560 Hull St., San Diego, CA 92152-5001)

Auditory/vocal response time was separated into perception time (PT) and movement time (MT) in trials with bottlenose dolphins (Tursiops truncatus) - two males and one female. Pressure catheters accepted into the nasal cavity by each dolphin recorded the pressure increase that preceded sound production. Time from acoustic stimulus onset to onset of pressure rise was recorded as PT (range 57 to $314 \mathrm{~ms}$ ) and pressure rise onset to dolphin sound onset was recorded as MT (range 63 to $363 \mathrm{~ms}$ ). Blindfolded dolphins trained to report a target by whistling often responded before completion of their 200- to 800-ms echolocation click trains. Detection of the target, indicated by whistling, before termination of the animal's own click train, suggests that dolphins do not voluntarily respond to each successive click but rather set a rhythm such that each click is emitted about $20 \mathrm{~ms}$ after the target echo arrives.

\section{8:40}

2aAB2. Interactions between commercial fishing and walleye pollock aggregations. Sarah Stienessen, Chris D. Wilson, and Anne B. Hallowed (Alaska Fisheries Sci. Ctr., Natl. Ocean. and Atmos. Admin., 7600 Sand Point Way, NE, Seattle, WA 98115, sarah.stienessen@noaa.gov)

Scientists with the Alaska Fisheries Science Center are conducting a multiyear field experiment off the eastern side of Kodiak Island in the Gulf of Alaska to determine whether commercial fishing activities significantly affect the distribution and abundance of walleye pollock (Theragra chalcogramma), an important prey species of endangered Steller sea lions (Eumetopias jubatus). In support of this activity, spatio-temporal patterns were described for pollock aggregations. Acoustic-trawl surveys were conducted in two adjacent submarine troughs in August 2001. One trough served as a control site where fishing was prohibited and the other as a treatment site where fishing was allowed. Software, which included patch recognition algorithms, was used to extract acoustic data and generate patch size and shape-related variables to analyze fish aggregations. Important patch related descriptors included skewness, kurtosis, length, height, and density. Estimates of patch fractal dimensions, which relate school perimeter to school area, were less for juvenile than for adult aggregations, indicating a more complex school shape for adults. Comparisons of other patch descriptors were made between troughs and in the presence and absence of the fishery to determine whether trends in pollock aggregation dynamics were a result of the fishery or of naturally occurring events.

\section{9:05}

2aAB3. Age-related hearing loss in sea lions and their scientists. Ronald J. Schusterman, Brandon Southall, David Kastak, and Colleen Reichmuth Kastak (Long Marine Lab., Univ. of California at Santa Cruz, 100 Shaffer Rd., Santa Cruz, CA 95060)

Interest in the hearing capabilities of California sea lions (Zalophus californianus) was first stimulated by the echolocation hypothesis and more recently by rising concern about coastal noise pollution. During a series of audiometric tests, we measured the absolute hearing sensitivity of two sea lions and two of their human investigators. Aerial hearing curves for each subject were obtained with a go/no-go procedure and standard psychophysics. Additionally, underwater hearing curves were obtained for the sea lions using 
the same procedures. Underwater, the older sea lion (22-25 years of age) showed hearing losses relative to the younger sea lion (13-16 years) that ranged from $10 \mathrm{~dB}$ at lower frequencies to $50 \mathrm{~dB}$ near the upper frequency limit. The older sea lions' hearing losses in air were consistent with those measured underwater. The older human (69 years) tested also showed losses relative to the younger human (22 years). These differences ranged from $15 \mathrm{~dB}$ at lower frequencies up to $35 \mathrm{~dB}$ at the highest frequency tested. The results obtained in this study document age-related hearing losses in sea lions and humans. The findings are consistent with data on presbycusis in other mammalian species, showing that maximum hearing loss occurs at the highest frequencies.

9:30

2aAB4. Interrelationships between intranarial pressure and biosonar clicks in the bottlenose dolphin (Tursiops truncatus). Wesley R. Elsberry (Marine Acoust. Lab., Texas A\&M Univ., Galveston, TX), Ted W. Cranford (San Diego State Univ., San Diego, CA), Sam H. Ridgway (SPAWAR Syst. Ctr., San Diego, CA), Donald A. Carder, William G. VanBonn (US Navy Marine Mammal Prog., San Diego, CA), Diane J. Blackwood (Marine Acoust. Lab., Texas A\&M Univ., Galveston, TX), Jennifer A. Carr (US Navy Marine Mammal Prog., San Diego, CA), and William E. Evans (Texas A\&M Univ., Galveston, TX)

Three Atlantic bottlenose dolphins (Tursiops truncatus) were given a target recognition biosonar task. During their performance of the task, both acoustic data in the far field and pressure within the bony nasal passages were digitally recorded (Elsberry et al., 1999). Analysis of over 15000 biosonar clicks provided new insights into odontocete biosonar sound production and is consistent with acoustic and pressure data taken from white whales (Delphinapterus leucas) during biosonar (Ridgway and Carder, 1988). Our work provides the first evidence for a minimum intranarial pressure during biosonar click production for any odontocete (11.8 \pm 0.5 $\mathrm{kPascals}$ over basal pressure). All three subjects exhibited nearly the same minimum tranarial pressure difference during biosonar click production. Clicks produced at or near this minimum intranarial pressure exhibited a wide range of acoustic power values. The acoustic power of a biosonar click was not highly correlated with intranarial pressure $\left(R^{2}=0.116\right)$. The radiated acoustic energy in biosonar clicks ranged from 1 to 1370 microJoules. Estimates of mechanical work during pressurization events were produced using a piston/cylinder model and intranarial volume data from prepared specimens and computed tomography scans. Mechanical work during pressurization events ranged from 2.74 to 23.0 Joules, with an average of 10.3 Joules.

9:55

2aAB5. Past and present research on gray whale vocalizations. Francisco Ollervides (RARE Ctr. for Tropical Conservation, 1840 Wilson Blvd., Ste. 402, Arlington, VA 22201-3000, follervides@ rarecenter.org)

Eberhadt and Evans first recorded vocalizations of gray whales in 1967 in Laguna Ojo de Liebre, Mexico. Three and a half decades of bioacoustic research on gray whales has followed this groundbreaking work. Gray whales appear more vocal while at the southern breeding lagoons off Baja California and are least vocal at the northern feeding areas of the Bering, Chukchi, and Beaufort Seas. Ten different vocalizations have been identified on the breeding grounds, while only six different vocalizations have been recorded on the feeding grounds. Evans also conducted the first recordings of vocalizations of gray whales in captivity with his work on J.J. II in San Diego in 1974. Since then, the database of captive gray whale vocalizations has expanded with research on GiGi in 1997. From this review, an acoustic repertoire of at least 12 different types of vocalizations is suggested. Nevertheless, not all gray whale calls fall into obvious or distinct categories; thus, correlating specific behaviors with each vocalization type merits further study.

10:20-10:30 Break

10:30

2aAB6. Do manatees talk during sex? Caryn Self-Sullivan (Texas A\&M Univ., College Station, TX 77843-2258), Tamra Gilbertson (Mote Marine Lab., Sarasota, FL 34236), and William E. Evans (Texas A\&M Univ., Galveston, TX 77553-1675)

On January 13, 1999, manatee vocalizations were recorded during a mating herd event in the Orange River, Florida. Although copulation could not be observed, multiple males were observed with exposed penises. During one 25 min sample (1300-1325 h), over 400 manatee signals were recorded. In March 2000, each signal was captured and digitized from the analog tape using a Marantz PMD 501, Ashly equalizer (gain=0, filter $=0$ ), MAC 8100, and Canary 1.2.1. In general, signals were 100-200 ms in length, highly harmonic (up to 8 harmonics ranging from 1 to $16 \mathrm{kHz}$ ), with little or no frequency modulation. Intervals between signals ranged from less than $1 \mathrm{~s}$ to $14 \mathrm{~s}$ (mean $=3 \mathrm{~s}$ ), indicating that manatees do indeed talk (a lot) during sex. Noise from two passing boats was also recorded during the sample period. One abnormally low-frequency signal $(0.4 \mathrm{kHz})$ was recorded during one boat pass. This apparent manatee vocalization could be seen and heard below the boat noise frequency band.

\section{0:55}

2aAB7. Echolocation signals of foraging killer whales (Orcinus orca). Whitlow W. L. Au (Hawaii Inst. of Marine Biol., Univ. of Hawaii, P.O. Box 1106, Kailua, HI 96734), John K. B. Ford (Pacific Biological Station, DFO Canada, Nanaimo, BC V6B 3X8, Canada), and Kelly A. Allman (The Marine Mammal Ctr., Sausalito, CA 94965)

Fish eating resident killer whales that frequent the coastal waters of Vancouver Island, Canada have a strong preference for chinook salmon. The whales in Johnston Strait often forage along the steep cliffs that extend into the water, echolocating their prey. Echolocation signals were measured with a four hydrophone symmetrical star array and the signals were simultaneous digitized at a sample rate of $500 \mathrm{kHz}$ using a lunch-box PC. A portable VCR recorded the images from an underwater camera located close to the array center. Only signals emanated from close to the beam axis (1185 total) were chosen for a detailed analysis. Killer whales project very broad band echolocation signals $(Q 1.3$ to 1.5$)$ that tend to have a bimodal frequency structure. Ninety seven percent of the 
signals had center frequencies between 45 and $80 \mathrm{kHz}$ with a band-width between 35 and $50 \mathrm{kHz}$. The peak-to-peak source level of the echolocation signal decreased as a function of the one way transmission loss to the array. Source levels varied between 200 and $225 \mathrm{~dB}$ re $1 \mu \mathrm{Pa}$. Using a model of target strength for chinook salmons, the echo levels from the echolocation signals are estimated for different ranges between whale and salmon.

\title{
Contributed Paper
}

\section{1:20}

2aAB8. Evoked potential application to study of echolocation in cetaceans. Alexander Ya. Supin (Inst. of Ecology and Evolution, Russian Acad. of Sci., 33 Leninsky Prosp., 119071 Moscow, Russia, a.supin@g23.relcom.ru), Paul E. Nactigall, Jeffrey Pawloski, and Whitlow W. L. Au (Hawaii Inst. of Marine Biol., Univ. of Hawaii, Kailua, HI)

The evoked-potential (EP) method is effective in studies of hearing capabilities of cetaceans. However, until now EP studies in cetaceans were performed only in conditions of passive hearing by recording EP to external stimuli. Can this method be applied to study active echolocation in odontocetes? To answer this question, auditory brainstem evoked re- sponses $(\mathrm{ABR})$ were recorded in a false killer whale while the animal echolocated a target within an experiment in which the animal reported the target present or absent. The ABR collection was triggered by echolocation clicks. In these conditions, the recorded ABR pattern contained a duplicate set of waves. A comparison of ABR wave delays recorded during echolocation with those recorded during regular external stimulation has shown that the first set of waves is a response to the emitted click whereas the second one is a response to the echo. Both responses, to the emitted click and to the echo, were of comparable amplitude in spite of the intensity difference of these two sounds of more than $40 \mathrm{~dB}$ near the animal's head. This finding indicates some mechanisms releasing responses to echoes from masking by loud emitted clicks. The evoked-potential method may be productive to investigate these mechanisms.

TUESDAY MORNING, 4 JUNE 2002

GRAND BALLROOM 3, 8:55 TO 11:35 A.M.

\section{Session 2aAO}

\section{Acoustical Oceanography and Underwater Acoustics: Acoustical Studies of Bubbles in the Ocean}

\author{
R. Lee Culver, Chair \\ Applied Research Laboratory, Pennsylvania State University, P.O. Box 30, State College, Pennsylvania 16804
}

Chair's Introduction-8:55

Invited Papers

9:00

2aAO1. Use of bubble cloud attenuation measurements to determine the bubble size distribution and comparison with backscatter measurements. Hugh Dumbrell (Offshore \& Acoust. Dept., QinetiQ Ltd., Bincleaves Technol. Park, Newtons Rd., Weymouth, Dorset DT4 8UR, UK, hadumbrell@qinetiq.com)

This paper presents the method of using bubble cloud attenuation measurements to determine the bubble size distribution, taking into account the effect of time-dependent bubble scatter and absorption. It then shows that the results of attenuation measurements due to bubble clouds (generated by a ship's wake) agree with backscatter measurements made at a much higher frequency.

9:20

2aAO2. Acoustic measurements of the number of naturally produced bubbles resonating at $\mathbf{5 0} \mathrm{kHz}$. Bernd Nuetzel and Karl-Heinz Herwig (FWG, Klausdorfer Weg 2-24, 24148 Kiel, Germany)

When the wind is strong enough to form whitecaps, bubbles caused by breaking waves penetrate into the water column and are transported to depths of more than 10 meters by Langmuir circulation, turbulence, and other mechanisms. In the near-surface layer, bubbles cause acoustic scattering and attenuation. Especially in shallow water, where the sound is reflected and scattered at the boundaries (sea bottom and surface), bubbles significantly influence sound propagation in the sea. The results from acoustic nearsurface experiments conducted in the North Sea over several years are presented. Data sets of acoustic attenuation were measured inside the bubble clouds for a propagation range of $1 \mathrm{~m}$. Also, a narrow-beam transducer was utilized to measure the acoustic scattering from the near-surface bubbles. Both investigations were done simultaneously for a large variety of environmental conditions. The data sets have been combined to determine the number of resonating bubbles as a function of depth and wind speed for a selected frequency of $50 \mathrm{kHz}$. A numerical expression is introduced for this special set of parameters. The comparison with results from other investigators using different techniques shows good agreement. 
2aA03. Laboratory studies on scattering from bubbles located close to an air-water interface. George Kapodistrias (Philips Medical Systems, Bothell, WA 98021, george.kapodistrias@philips.com) and Peter H. Dahl (Appl. Phys. Lab., Univ. of Washington, Seattle, WA 98105)

Results of laboratory experiments addressing the problem of scattering from bubbles located near an air-water interface are discussed. Well-controlled measurements of scattering from bubbles placed on a fine thread (itself nearly transparent to sound) were made. Depending on the experiment, bubble radii ranged from 500-1000 $\mu \mathrm{m}$, while insonifying frequencies ranged from 110-130 $\mathrm{kHz}$. The experiments quantified multiple scattering effects, by obtaining scattering data from two bubbles symmetrically arranged at a distance, $d$, from the acoustic beam axis, and the influence of both a flat and a roughened air-water interface, by measuring backscattering from a bubble located a distance, $d$, from the interface. Experiments were performed in the $k d$ range $0.2-21$, where $k$ is the acoustic wave number. Modeling was achieved by utilizing the multiple scattering series and ray synthesis of the four scattering paths, created by the proximity of the bubbles to the interface, while addressing both bistatic and monostatic conditions. Experimental results are in good agreement with simulations, and they highlight three important features of the problem of acoustic observations from subsurface bubbles: (1) multiple propagation paths, (2) multiple scattering, and (3) rough surface effects. The relation of this work to interpretive models of field data is also discussed.

\section{0:00}

2aA04. The acoustics of bubbles and bubbly assemblages: a suite of laboratory studies. Ronald A. Roy, Preston S. Wilson, Ryan D. McCormick, Eun-Joo Park (Dept. of Aerosp. and Mech. Eng., Boston Univ., Boston, MA 02215, ronroy@bu.edu), and William M. Carey (Boston Univ., Boston, MA 02215)

The in situ study of oceanic bubble acoustics is a challenging endeavor. One must impose a variety of a priori assumptions and constraints in order to extract information that unambiguously sheds light on the underlying physical mechanisms that govern the transmission and scattering of sound in oceanic bubble assemblages. Conversely, laboratory studies afford an added measure of physical control and observational access that permits one to focus on certain facets of bubble activity with accuracy and precision. We provide an overview of several laboratory-based bubble acoustics studies under way at Boston University. The scope of the work spans the spectrum from the quantitative measurement of acoustic dispersion through, and scattering from, high void fraction bubbly regions, to detailed studies of the linear dynamics of individual bubbles. [Work supported by ONR.]

\section{0:20-10:35 Break}

\section{Contributed Papers}

\section{0:35}

2aAO5. Measuring bubble distribution with multibeam sonar. R. Lee Culver and David Bradley (Appl. Res. Lab., The Penn State Univ., P.O. Box 30, State College, PA 16804, rlc5@psu.edu)

Bubbles in water are very efficient scatterers of sound. Depending upon the ratio of acoustic wavelength to bubble radius, the scattering mechanism can be Rayleigh, resonant, or geometric. The strength of the scattered signal depends strongly on this ratio. The strength of the scattered signal can be used to estimate the number of bubbles per unit volume, or bubble density, if the size of the bubbles or the size distribution of the bubbles is known. A number of researchers have employed narrow beam sonar to measure the density of bubbles in water. We have used a $250 \mathrm{kHz}$ sonar with 19 narrow beams, integrated into an autonomous vehicle (AUV), to image the wakes of two large surface ships from the underside. An earlier talk focused on the structure of the turbulent wake revealed by the measurements [J. Acoust. Soc. Am. 110, 2642 (2001)]. In this talk we discuss parameters and assumptions which strongly affect the bubble density estimate. These include: values for the transmit and receive beamwidth; the shape of the bubble size distribution spectrum, including possible variation with depth; strong boundary reflections and high sidelobe levels; and the possible influence of multiple scattering. [Work sponsored by ONR Code 321OA.]

\section{0:50}

2aAO6. Multibeam echo-sounding measurement of the microbubble field in a ship's wake. Timothy C. Gallaudet (Marine Physical Lab., Scripps Inst. of Oceanogr., 9500 Gilman Dr., La Jolla, CA 92093-0205) and Christian P. de Moustier (Univ. of New Hampshire, Durham, NH 03824)

Acoustic data collected with the U.S. Navy's $68 \mathrm{kHz}$ Toroidal Volume Search Sonar (TVSS) system were used to measure the microbubble field generated by the wake of the towing vessel at a constant distance of 18 ship lengths astern. Swath bathymetry beamforming and echo detection techniques were adapted to map the sea surface and the spatial distribution of bubble fields observable in the ocean volume with the TVSS. The sonar was towed at $78 \mathrm{~m}$ depth yielding a sea surface measurement swath roughly $550 \mathrm{~m}$ wide thus allowing observations of wake bubble fields across three parallel ship tracks spaced $200 \mathrm{~m}$ apart and over $22 \mathrm{~min}$ after generation. Measurements in microbubble fields about $3 \mathrm{~min}$ after generation by the ship's wake yielded an average vertical attenuation of $2.1 \mathrm{~dB} / \mathrm{m}$ and a maximum acoustic volume scattering strength of $-17.1 \mathrm{~dB}$. [Work supported by the U.S. Naval Research Laboratory.]

\section{1:05}

2aA07. Characteristics of open ocean bubble plumes from a 3-month inverted echo-sounder record. Mark V. Trevorrow (Defence Res. Establishment Atlantic, P.O. Box 1012, Dartmouth, NS B2Y 3Z7, Canada)

A self-contained $200-\mathrm{kHz}$ inverted echo-sounder was deployed at Ocean Station Papa (NE Pacific, $1400 \mathrm{~km}$ west of Vancouver Is.) from 1 March to 20 May, 1996. The instrument operated continuously, recording calibrated volume scattering from near-surface bubbles with 3-s and $30-\mathrm{cm}$ resolution. Average surface wind and significant wave height during this period were $7.5 \mathrm{~m} / \mathrm{s}$ and $2.8 \mathrm{~m}$, respectively. The data show the frequent occurrence of bubbles organized into vertical, plume-like structures, presumably drawn downward within the convergence zones of Langmuir circulation. Significant bubble plume activity (defined as average plume depth $>3 \mathrm{~m}$ ) was observed during $61 \%$ of the operating period. Average bubble plume penetrations of up to $15 \mathrm{~m}$ were observed, with maximum penetrations up to $25 \mathrm{~m}$. Within the plumes, the backscatter cross section exhibited an exponential decay with depth, with e-folding scale in the range 0.5 to $2.5 \mathrm{~m}$. Some implications of this plume-like structure for high-frequency sonar performance will be discussed. 
tenuated and dispersive. Theory to explain this behavior exists in the lit-

2aA08. Measurements of the time-dependent attenuation in a nonstationary bubble distribution. Preston S. Wilson, Ronald A. Roy, and William M. Carey (Dept. of Aerosp. and Mech. Eng., Boston Univ., Boston, MA 02115)

In the ocean, natural and artificial processes generate clouds of bubbles which scatter and attenuate sound, and the structure of these clouds (i.e., the space-dependent bubble size distribution and void fraction) evolves over time. Measurements have shown that at the individual bubble resonance frequency (IBRF), sound propagation in this medium is highly at- erature and is experimentally verified away from resonance. However, due to excessive attenuation near resonance and the high degree of sensitivity to bubble population parameters, little adequate experimental data exists for comparison and theory remains largely unverified. Using an impedance tube, the time-dependent attenuation has been measured in a nonstationary laboratory bubbly fluid at IBRF, along with measurements of the accompanying time-dependent bubble population parameters. The relative variability of the measured maximum attenuation and the frequency at which it occurred agreed closely with existing theory. [Work supported by ONR.]

TUESDAY MORNING, 4 JUNE 2002

BRIGADE ROOM, 8:00 TO 10:05 A.M.

Session 2aEA

\title{
Engineering Acoustics and Architectural Acoustics: Electroacoustic Systems for 3-D Audio
}

\author{
Gary W. Elko, Chair \\ MH Acoustics, 26 Blackburn Place, Summit, New Jersey 07901
}

Chair's Introduction-8:00

Invited Papers

8:05

2aEA1. Measurement of 3-D directional characteristics of reverberant sound fields using a spherical microphone array. Bradford N. Gover (Inst. for Microstructural Sci., Natl. Res. Council, Ottawa, ON K1A 0R6, Canada, bradg@ @audiolab.uwaterloo.ca)

A new measurement system has been used to analyze directional characteristics of reverberant sound fields in several small- and medium-sized rooms. The system employs a pair of 32-element spherical microphone arrays to obtain the room impulse response in each of 60 steering directions. The two different-sized arrays are used to cover the frequency range from $300-3300 \mathrm{~Hz}$ with an angular (directional) resolution of about 28 degrees. An analysis of these directional room impulse responses enables the variation of arriving sound energy with (3-D) direction and time to be studied. Specifically, the isotropy of the sound field at the receiving position can be assessed at any time instant, or over any time range desired. Directions from which significant or insignificant proportions of sound energy arrive are identifiable. Furthermore, the time and direction of incidence of the direct sound and discrete early reflections can be identified. This can be of use in localizing sources, finding reflections, and in decomposing the sound field into arriving components.

$8: 35$

2aEA2. A spherical microphone array for spatial sound recording. Jens Meyer and Gary W. Elko (mh acoustics, jm@mhacoustics.com,gwe@mhacoustics.com)

The progression of audio from monophonic to the present day 5-channel playback is being driven by the desire to improve the immersion of the listener into the acoustic scene. In the limit, the goal is the reconstruction of the original sound field. This talk describes the decomposition of the sound field into orthogonal components, the so-called spherical harmonics and is directly related to the method of Ambisonics. These components contain all required information to allow a reconstruction of the original sound field. This approach is scalable to any number of loudspeakers and is also backwards compatible to surround sound, stereo and mono playback. One problem is the recording of the orthogonal components. So far only solutions exist that allow the recording of spherical harmonics up to first order. This limits the spatial resolution. This presentation introduces a new microphone that overcomes this limitation. It consists of pressure sensors that are equally distributed on the surface of a rigid sphere. The number of sensors depends on the highest order spherical harmonic to be recorded. A minimum of $(n+1)^{2}$ sensors is required to record harmonics up to $n$th order. The sensor signals are then processed to give the desired spherical harmonic outputs.

\section{9:05}

2aEA3. Control transducer locations for virtual acoustic imaging using binaural principle. Takashi Takeuchi (Bldg. Environment Group, Bldg. Eng. Dept., Kajima Tech. Res. Inst., 2-19-1 Tobitakyu, Chofu-shi Tokyo 182-0036, Japan) and Philip A. Nelson (Univ. of Southampton, England)

When binaural sound signals are presented with loudspeakers, the systems inversion involved gives rise to a number of problems such as loss of dynamic range, deterioration of control performance by small errors, and room reflections. A method of overcoming these fundamental problems is proposed. A conceptual monopole transducer is introduced whose azimuthal position varies continuously as frequency varies. This gives a minimum processing requirement of the binaural signals for the control to be achieved and all 
the above problems either disappear or are minimized. Among a number of practical solutions to realize such optimally distributed transducers is a discretization that enables the use of conventional transducer units. As for the elevation location of the control transducers, the analysis of the spectral cues and dynamic cues is performed. The frequency response of the plant that relates transducer outputs to ear-pressure signals suggests that control transducer positions will be promising at positions in the frontal plane above the listener's head. The analysis of the dynamic cues induced by unwanted head rotation also strongly supports the use of transducer locations in the frontal plane. Subjective experiments are performed and the methods described here are proved to be advantageous.

\title{
9:35
}

2aEA4. Audio recording and reproduction in CARROUSO: Getting closer to perfection? Heinz Teutsch, Sascha Spors, Herbert Buchner, Rudolf Rabenstein, and Walter Kellermann (Chair of Multimedia Commun. and Signal Processing, Univ. of Erlangen-Nuremberg, Cauerstr. 7, 91058 Erlangen, Germany)

State-of-the-art systems for spatial audio reproduction utilize two to six discrete playback channels. A problem inherent to these systems is the relatively small area where the listener is able to experience a true 3-D sound sensation. This so-called "sweet spot" can be significantly enlarged by using loudspeaker arrays in combination with wave field synthesis (WFS) technology, initially developed at Delft University. By following this approach, actual sonic spaces can be reproduced in their entirety and not only discrete multichannel representations thereof. While loudspeaker arrays can be used to reproduce sound fields, microphone arrays can be used for sound field capture and analysis. Having high-quality audio reproduction in mind, microphone array designs are presented that need to fulfill stricter requirements than what has been traditionally considered for microphone array applications. Information on acoustic source position is essential for WFS-based rendering techniques. As will be shown, joint audio-video object tracking proves to be efficient for this task. Moreover, full-duplex applications based on WFS technology, like high-quality teleconferencing or remote music teaching, call for sophisticated multichannel acoustic echo cancellation algorithms. The European project "CARROUSO" aims at developing, integrating, and building a real-time system that embraces all previously described technologies in an MPEG-4 context.

\section{Session 2aED}

\section{Education in Acoustics: Hands-on Demonstrations in Acoustics for High School Students}

\author{
Uwe J. Hansen, Chair \\ Department of Physics, Indiana State University, Terre Haute, Indiana 47809
}

\begin{abstract}
Approximately 20 experiments designed to introduce various acoustics principles will be set up in the room. After a brief introduction of each demonstration, they will be available for experimentation by visiting high school students. Space permitting, conference participants are also invited to view the demonstrations and perform hands-on experiments, provided such activity does not interfere with the purpose of the session, i.e. to introduce acoustics experiments to high school students. Experiments performed by the students will range in sophistication from simple wave studies to mapping of normal modes in two-dimensional structures.
\end{abstract}

TUESDAY MORNING, 4 JUNE 2002

RIVERS ROOM, 8:25 TO 11:30 A.M.

\section{Session 2aMU}

\section{Musical Acoustics: Interactive Computer Music Systems}

\author{
Roger Dannenberg, Chair \\ School of Computer Science, Carnegie Mellon University, Pittsburgh, Pennsylvania 15213 \\ Chair's Introduction-8:25 \\ Invited Papers
}

8:30

2aMU1. The structural implications of interactive creativity. Joel Chadabe (Electron. Music Foundation, 116 N. Lake Ave., Albany, NY 12206, chadabe@aya.yale.edu)

The functioning of any particular electronic musical instrument can be placed somewhere along a line that extends from deterministic to indeterministic. Although deterministic instruments may offer more powerful controls than traditional instruments, they typically put a performer in the traditional situation of making a gesture and expecting a predictable effect. Indeterministic instruments, on the other hand, put a performer in an interactive role of improvising relative to an unpredictable output. The unique 
advantage of such interactive instruments is that they foster "interactive creativity." The design of a traditional instrument is fundamentally different from the design of an interactive instrument. A traditional instrument is structured as a single cause and effect, articulated as a synchronous linear path through a hierarchy of controls from a performer operating an input device to the multiple variables of a sound generator. An interactive instrument, on the other hand, is structured as a network of many causes and effects at various levels of importance, with a performer's input as only one of the causes of the instrument's output in sound. The author will present several historical examples of interactive electronic musical instruments and offer some speculations on the future.

\section{9:00}

2aMU2. Live interactive computer music performance practice. David Wessel (Ctr. for New Music and Audio Technologies (CNMAT), Dept. of Music, Univ. of California, Berkeley, Berkeley, CA 94720, wessel@cnmat.berkeley.edu)

A live-performance musical instrument can be assembled around current lap-top computer technology. One adds a controller such as a keyboard or other gestural input device, a sound diffusion system, some form of connectivity processor(s) providing for audio I/O and gestural controller input, and reactive real-time native signal processing software. A system consisting of a hand gesture controller; software for gesture analysis and mapping, machine listening, composition, and sound synthesis; and a controllable radiation pattern loudspeaker are described. Interactivity begins in the set up wherein the speaker-room combination is tuned with an LMS procedure. This system was designed for improvisation. It is argued that software suitable for carrying out an improvised musical dialog with another performer poses special challenges. The processes underlying the generation of musical material must be very adaptable, capable of rapid changes in musical direction. Machine listening techniques are used to help the performer adapt to new contexts. Machine learning can play an important role in the development of such systems. In the end, as with any musical instrument, human skill is essential. Practice is required not only for the development of musically appropriate human motor programs but for the adaptation of the computer-based instrument as well.

\section{9:30}

2aMU3. The IMUTUS interactive music tuition system. George Tambouratzis, Stelios Bakamidis, Ioannis Dologlou, George Carayannis, and Markos Dendrinos (Inst. for Lang. and Speech Processing, 6 Artemidos Str. \& Epidavrou, 15125 Paradissos Amaroussiou, Greece)

This presentation focuses on the IMUTUS project, which concerns the creation of an innovative method for training users on traditional musical instruments with no MIDI (Musical Instrument Digital Interface) output. The entities collaborating in IMUTUS are ILSP (coordinator), EXODUS, SYSTEMA, DSI, SMF, GRAME, and KTH. The IMUTUS effectiveness is enhanced via an advanced user interface incorporating multimedia techniques. Internet plays a pivotal role during training, the student receiving guidance over the net from a specially created teacher group. Interactiveness is emphasized via automatic-scoring tools, which provide fast yet accurate feedback to the user, while virtual reality methods assist the student in perfecting his technique. IMUTUS incorporates specialized recognition technology for the transformation of acoustic signals and music scores to MIDI format and incorporation in the training process. This process is enhanced by periodically enriching the score database, while customization to each user's requirements is supported. This work is partially supported by European Community under the Information Society Technology (IST) RTD programme. The authors are solely responsible for the content of this communication. It does not represent the opinion of the European Community, and the European Community is not responsible for any use that might be made of data appearing therein.

10:00

2aMU4. Machine musicianship. Robert Rowe (New York Univ., 35 W. Fourth St., Rm. 777, New York, NY 10012, robert.rowe@nyu.edu)

The training of musicians begins by teaching basic musical concepts, a collection of knowledge commonly known as musicianship. Computer programs designed to implement musical skills (e.g., to make sense of what they hear, perform music expressively, or compose convincing pieces) can similarly benefit from access to a fundamental level of musicianship. Recent research in music cognition, artificial intelligence, and music theory has produced a repertoire of techniques that can make the behavior of computer programs more musical. Many of these were presented in a recently published book/CD-ROM entitled Machine Musicianship. For use in interactive music systems, we are interested in those which are fast enough to run in real time and that need only make reference to the material as it appears in sequence. This talk will review several applications that are able to identify the tonal center of musical material during performance. Beyond this specific task, the design of real-time algorithmic listening through the concurrent operation of several connected analyzers is examined. The presentation includes discussion of a library of $\mathrm{C}++$ objects that can be combined to perform interactive listening and a demonstration of their capability. 
2aMU5. Making the computer "listen" to music. Christopher S. Raphael (Dept. of Mathematics and Statistics, Univ. of Massachusetts, Amherst, MA 01002-4515)

A computer system is discussed that provides real-time accompaniment to a live musician playing a non-improvisatory piece of music. Particular attention is devoted to the "listening" process, in which the computer must follow the soloist's progress through the musical score by interpreting the sampled acoustic signal. The process is complicated by the significant variation and occasional errors from the live player during performance. A hidden Markov model is introduced providing a principled, trainable, and fast solution to the listening problem. The system is capable of assessing its own level of uncertainty about score position, as well as accommodating the sometimes strong signal component from the accompaniment instrument. A live demonstration will be provided.

\title{
11:00
}

2aMU6. Music scene description: Toward audio-based real-time music understanding. Masataka Goto ("Information and Human Activity,” PRESTO, JST. / AIST (former ETL)., 1-1-1 Umezono, Tsukuba, Ibaraki 305-8568, Japan, m.goto@aist.go.jp)

Music understanding is an important component of audio-based interactive music systems. A real-time music scene description system for the computational modeling of music understanding is proposed. This research is based on the assumption that a listener understands music without deriving musical scores or even fully segregating signals. In keeping with this assumption, our music scene description system produces intuitive descriptions of music, such as the beat structure and the melody and bass lines. Two real-time subsystems have been developed, a beat-tracking subsystem and a melody-and-bass detection subsystem, which can deal with real-world monaural audio signals sampled from popular-music CDs. The beat-tracking subsystem recognizes a hierarchical beat structure comprising the quarter-note, half-note, and measure levels by using three kinds of musical knowledge: of onset times, of chord changes, and of drum patterns. The melody-and-bass detection subsystem estimates the $F 0$ (fundamental frequency) of melody and bass lines by using a predominant- $F 0$ estimation method called PreFEst, which does not rely on the $F 0$ 's unreliable frequency component and obtains the most predominant $F 0$ supported by harmonics within an intentionally limited frequency range. Several applications of music understanding are described, including a beat-driven, real-time computer graphics and lighting controller.

TUESDAY MORNING, 4 JUNE 2002

DUQUESNE ROOM, 9:00 TO 10:45 A.M.

\section{Session 2aNS}

\section{Noise, Architectural Acoustics and Structural Acoustics and Vibration: Acoustics of Glazing and of Urban Spaces}

\author{
Gregory C. Tocci, Chair \\ Cavanaugh Tocci Associates, 327F Boston Post Road, Sudbury, Massachusetts 01776
}

Chair's Introduction-9:00

Invited Papers

9:05

2aNS1. Analytic modeling of monolithic, laminated, and insulating glass sound transmission loss. Gregory Tocci (Cavanaugh Tocci Assoc., Inc., 327F Boston Post Rd., Sudbury, MA 01776)

The sound transmission loss of single lights of glass, and of insulating glass configurations using various combinations of monolithic and laminated glass, have been computed using Mathcad, and formulations of sound transmission loss as described by Beranek and Ver, and Au and Byrne. The importance of damping in these formulations, as seen by comparing estimated and measured TLs, is discussed. This discussion is extended to demonstrate the need for unifying methods for mounting glass and window samples in laboratory test openings, and to suggest ways to do so. [Work supported by Solutia, Inc.] 
2aNS2. Sound isolation performance of interior acoustical sash. Gregory Tocci (Cavanaugh Tocci Assoc., Inc., 327F Boston Post Rd., Sudbury, MA 01776)

In existing, as well as new buildings, an interior light of glass mounted on the inside of a prime window is used to improve the sound transmission loss otherwise obtained by the prime window alone. Interior acoustical sash is most often 1/4 in. (6 mm) monolithic or laminated glass, and is typically spaced 3 in. to 6 in. from the glass of the prime window. This paper presents TL data measured at Riverbank Acoustical Laboratories by Solutia (formerly Monsanto) for lightweight prime windows of various types, with and without interior acoustical sash glazed with $1 / 4 \mathrm{in}$. laminated glass. The TL data are used to estimate the A-weighted insertion loss of interior acoustical sash when applied to prime windows glazed with lightweight glass for four transportation noise source typeshighway traffic, aircraft, electric rail, and diesel rail. The analysis also has been extended to determine the insertion loss expressed as a change in OITC. The data also exhibit the reductions in insertion loss that can result from short-circuiting the interior acoustical sash with the prime window. [Work supported by Solutia, Inc.]

\section{Contributed Papers}

\section{9:45}

2aNS3. Practical problems of sound isolation through interior glazing systems. Carl Rosenberg and Dorie Najolia (Acentech, Inc., 33 Moulton St., Cambridge, MA 02138)

In a number of recent projects, designers have expanded the concepts of visual openness within a building to incorporate large expanses of glass. At the same time, building occupants expect the glass to achieve similar levels of acoustical isolation as stud constructed partitions. These situations include glass-enclosed conference rooms, clerestory lites between offices and corridors, curtain walls and strip windows, and vision panels that are adjacent to large atrium spaces. Variables impacting the acoustical performance include the size of the vision panels, the type of glass, the airspace between multiple layers, and the framing system that holds the glass. This paper presents a number of case studies and methodologies that address these issues.

10:00

2aNS4. The concept of privacy in the social practice of the living environment through sonic phenomena (case of the old city of Constantine. Algeria). Derbal Cobis Rabah and Zeghlache Hamza (BP.12.SMK. Constantine 25003. Algeria. cobis-derbal@iquebec.com)

The privacy of the different aspects composing the outside space and its social practice in the medinas and old islamic cities, is found to be often modulated by the quality of the physical criteria of the environment. Different activities in the medina have greatly modified the privacy suroundings. Privacy has been therefore, shaken up and greater permeability between inside and outside, particularly through sound phenomena, is one of the characteristics of such space. The exterior sound environment is fully perceived despite the supposed dispositions to preserve a certain degree of privacy. Underway studies on the old city of Constantine show already a great need to reconsider the concept of privacy in urban area throughout the sound environment. The noise acts, most of the time, not only by its disturbance, but by its information content as well, which is supposed to reduce and weaken significantly the concept of privacy in such old cities. More work is to be considered involving multidisciplinary approaches so that the notion of privacy can be worked out in reforming the discomfort of sonic environment into easy using soundscape with which privacy through social and historical mutations would fit in well.
10:15

2aNS5. Noise dimension in the design of urban spaces: Sonic instrumentation of public places. Derbal Cobis Rabah (BP. 12, SMK, Constantine 25003, Algeria, cobis-derbal@iquebec.com)

The sonic environment dimension is not often taken into account in the design of urban spaces. This is not the same for internal spaces inside which a certain control of the quality of the sonic conditions is henceforth possible. However in the case of urban spaces, one is often confronted with situations complex and difficult to handle. This always leads to an impredictible situation in terms of noise and urban sonic environment. Underway studies have shown the importance of the quality of the urban sonic environment in the daily use of public places. The latter, as elements that mark strongly the degree of dynamism of the urban structure of our cities, are often subjected to a very complex situation of noise pollution. Introducing a source of noise that might have an effect of recomposing positively the sonic environment of the public place was the main approach of the study. It can be used as well to restore the sound in its utilitarian and practical function and to articulate the perception of the spatial and temporal aspects of the place. It is a sort of a sonic instrumentation of urban spaces that might recompose or recharacterize urban and architectural environments.

\section{$10: 30$}

2aNS6. A multidisciplinary approach of the problem of noise nuisance in urban environment. Derbal Cobis Rabah and Zeghlache Hamza (BP. 12, SMK, Constantine 25003, Algeria, cobis-derbal@iquebec.com)

More often the problem of noise and sonic pollution, particularly in urban sites, is studied by different disciplines such as physics, the acoustics, the psychoacoustics, the medicine and others. It is independently of each other that these sciences are often approaching this subject. Some studies are carried out in laboratories taking noise as samples cut off their realistic context. Urban noise is studied as well by making an abstraction of the different contextual parameters by idealizing a rather complex sonic environment. The noise, according to this present approach, is suposed to react with surounding space, and it takes the form and the quality of the place by defining and requalifying it. It is found that the contextual aspects such as social, cultural or even symbolic dimensions modulate the listening conditions and the perception quality of the noise and even the living and the daily practice of the urban space. The multiparameter dimension study of the noise in an urban context is necessary to better work out the problem and to try to come up with some practical and efficient solutions. The little amount of studies based on such multidisciplinary approach, confort well our effort to go ahead with this methodological approach. 


\title{
Session 2aPA
}

\section{Physical Acoustics and Underwater Acoustics: Recent Developments in Wave Propagation in Random and Complex Media I}

\author{
D. Keith Wilson, Chair \\ AMSRL CI EE, U.S. Army Research Laboratory, 2800 Powder Mill Road, Adelphi, Maryland 20783-1197
}

Chair's Introduction-8:25

Invited Papers

$8: 30$

2aPA1. Quasiwavelet models of sound scattering by atmospheric turbulence. George H. Goedecke (Dept. of Phys., New Mexico State Univ., Las Cruces, NM 88003, ggoedeck@ nmsu.edu), Vladimir E. Ostashev (New Mexico State Univ., Las Cruces, NM 88003 and NOAA/Environ. Tech. Lab., Boulder, CO), D. Keith Wilson, and Harry J. Auvermann (U.S. Army Res. Lab., Adelphi, MD)

Quasiwavelet $(\mathrm{QW})$ representations of turbulence are composed of self-similar, localized, eddylike structures. The QW functions are not true wavelets, in that they do not form a mathematically complete basis or have zero mean. Nevertheless, they appear to be very useful for applications involving scattering and propagation of sound waves. In this paper, the QW formulation of Goedecke and Auvermann [J. Acoust. Soc. Am. 102, 759-771 (1997)] is outlined. The QW expressions for the spatial spectra and the corresponding sound scattering cross sections due to the velocity and temperature fluctuations of isotropic homogeneous turbulence are discussed. The spectra for different eddy structures are always similar to the von Karman spectra, and agree with the Kolmogorov spectra in the inertial range. Equations that yield the QW eddy functions in terms of the spectra are derived, and a QW function is found that yields the von Karman velocity spectrum exactly. Some results are presented from a numerical calculation of coherent scattering and temporal spectral broadening due to advecting turbulence modeled by QW eddies flowing with a wind. Future applications to modeling scattering by anisotropic and/or inhomogeneous turbulence are discussed. [Work supported by the ARO under Contract No. DAAD19-01-1-0640 (administered by W. Bach).]

8:50

2aPA2. Some validity issues in the theory and modeling of WPRM. Terry Ewart and Frank Henyey (Univ. of Washington, M.S. 355640, Seattle, WA 98105)

In many publications in the recent to older litererature on the theory and modeling of wave propagation in random media, WPRM, in ocean and atmospheric environments there are serious validity issues that arise. The issues discussed will include the following. (1) The validity of using the Markov approximation in theoretical treatments must be established for specific environments before theories based on that approximation can be used. Parameters that are required to be small must be shown to be so before the applicabilty to specific environments can be assured. (2) Most publications that purport to model the pdf's of intensity do not include the standard "goodness of fit" parameters. The Kolmogorov-Smirnov GoF test emphasizes the center of the distribution and often the pdf's of WPRM are characterized by very high tailed distributions where the fitting of that component can be important to understanding the scattering physics. GoF tests that include the full range of received intensity are needed. (3) Signal processors invariably require that the quadrature components of their receptions have Gaussian pdf's. It has been shown by simulations of WPRM that this is often not even close to the case. What significance this effect has on signal processing algorithms is a difficult and unresolved issue.

9:10

2aPA3. Recent results from basin-scale ocean acoustic wave propagation: Implications for the general theory of the approach to saturation. John A. Colosi (Woods Hole Oceanogr. Inst., M.S. 11, Woods Hole, MA 02543)

Long-range ocean acoustics as a problem in wave propagation through random media is unique in several ways. First the propagation takes place in the ocean sound channel (a waveguide), and second, a primary source of sound speed fluctuations are the anisotropic and inhomogeneous ocean internal waves (not turbulence). Recent observations of $75 \mathrm{~Hz}$, broadband transmissions over several thousand kilometers have revealed fascinating results on the approach to saturation. Two distinct propagation regimes are observed in the same pulse: a fully saturated regime characterized by Rayleigh statistics, and an unsaturated regime in which the probability density function for intensity is nearly log-normal. The transition between these two regimes is extremely rapid. The saturated region of the pulse is composed of acoustic energy traveling near the waveguide axis, while the unsaturated region is composed of acoustic energy strongly refracted by the waveguide. This fact suggests a fundamental stabilizing influence by the waveguide. Application of path-integral theory, which was successful in describing shorter-range ocean experiments, cannot describe these two regimes. Recent efforts using semiclassical and ray chaos theory have led to new insights into the observational results and to the failure of the path-integral theory, yet a complete theoretical description remains elusive.

9:30-9:45 Break 


\section{Contributed Papers}

9:45

2aPA4. An eigenfunction expansion for low-frequency acoustic propagation in a downward-refracting stratified medium over a complex impedance plane. Roger Waxler (NCPA, Coliseum Dr., University, MS 38677-1848, rwax@olemiss.edu)

The well-known eigenfunction expansions associated with self-adjoint operators cannot be used to describe sound propagation over a complex impedance plane since, if the impedance has a real part, the resulting impedance boundary condition is not self-adjoint. Instead, a less widely known eigenfunction expansion associated with non-self-adjoint operators can be used. This eigenfunction expansion is applied to a vertically stratified model for downward refraction in the nighttime boundary layer. As in the self-adjoint case the propagation separates into a ducted part, expressed as a sum of modes which decay exponentially with height, and an upwardly propagating part, expressed as an integral over modes which are asymptotically (with height) plane waves. The eigenvalues associated with the ducted modes are complex, the imaginary parts being related to the acoustic attenuation. An efficient method for finding the complex eigenvalues is introduced from which a physically intuitive form of the attenuation coefficients is obtained. For low-frequency propagation $(100 \mathrm{~Hz}$ or less) the number of modes is small, making this a simple way to model the ducted part of the propagation.

\section{0:00}

2aPA5. Including attenuation and dispersion in time domain modeling of broadband sound propagation in dispersive oceanic media. Guy V. Norton (Naval Res. Lab., Stennis Space Center, MS 39529-5004, norton@nrlssc.navy.mil) and Jorge C. Novarini (Planning Systems, Inc., Long Beach, MS 39560)

Shallow water sound propagation almost invariably involves paths through regions occupied by assemblages of microbubbles near the air/ water interface and through the underlying sediment. Such an environment is intrinsically dispersive. Any reliable numerical model of acoustic propagation in this type of environment requires the inclusion of attenuation and its causal companion, dispersion. The time domain is the natural domain for numerical modeling of broadband pulse propagation. The inclusion of causal attenuation and dispersion within a numerical time domain model has in the past presented a challenge to modelers. For the case of propagation in a linear medium, Szabo [T. L. Szabo, J. Acoust. Soc. Am. 96, 491-500 (1994)], making use of generalized functions, introduced the concept of a convolutional propagation operator that plays the role of a causal propagation factor in the time domain. Utilizing a Finite Difference Time Domain (FDTD) model it is shown that the inclusion of the convolutional propagation operator correctly carries the information of attenuation and dispersion into the time domain. The causal FDTD model was exercised on environments having functional forms for the attenuation of the type encountered at sea, both in typical bubble clouds as well as the underlying sediment. [Work supported by ONR/NRL.]

\section{0:15}

2aPA6. Ultrasonic b-scan imaging with adaptive beamformation using aberration correction. Wayne C. Pilkington (Dept. of Elec. and Computer Eng., Univ. of Rochester, Rochester, NY 14627, pilkingt@ece.rochester.edu), James C. Lacefield (Depts. of Elec. and Computer Eng. and Medical Biophys., Univ. of Western Ontario, London, ON N6A 5B9, Canada), and Robert C. Waag (Depts. of Elec. and Computer Eng. and Radiol., Univ. of Rochester, Rochester, NY 14627)

The effectiveness of adaptive beam formation using aberration correction has been demonstrated in ultrasonic b-scans of liver-mimicking scattering phantoms imaged with and without an intervening aberrator that produced wavefront distortion comparable to that of abdominal wall. Images of $4 \mathrm{~mm}$ diam spherical features (either positive or negative contrast lesions or scatterer-free cysts) in the uniform scattering background of the phantoms were produced at $3.0 \mathrm{MHz}$ with a two-dimensional (80×80-element) array transducer system. Time-shift aberration was estimated from the scattering data and used to compensate both transmit and receive waveforms. Image improvements were assessed by comparison of feature contrast with and without aberration correction in individual images and by comparison of intensities in averages of independent, statistically identical images. Feature contrasts and borders were visibly and measurably improved, sometimes to near the water path results, using aberration correction, particularly when both transmit and receive corrections were applied. An efficient implementation of aberration correction was achieved by correction of multiple image scan lines with a single aberration estimate. Aberration correction using estimates from oneseventh the number of scan lines in $8 \mathrm{~mm}$ wide images produced improvements comparable to those achieved by individually estimating and correcting aberration in every scan line.

\section{0:30}

2aPA7. Statistical estimation of propagation path parameters for ultrasonic aberration correction. Robert C. Waag (Depts. of Elec. and Computer Eng. and Radiol., Univ. of Rochester, Rochester, NY 14627) and Jeffrey P. Astheimer (66 Sibley Rd., Honeoye Falls, NY 14472)

Coefficients of a finite impulse response linear filter model for ultrasonic propagation are found using a statistical estimation. The model employs a Green's function to describe scattering received by a twodimensional array from a random-medium volume illuminated by a transmit beam. The frequency response of this Green's function is factored into a homogeneous-transmission term and a path-dependent aberration term. Relative amplitude and phase of the aberration response are estimated over the frequency band of the transmit-receive system by using scattering from closely situated volumes and assuming that the aberration is the same for each volume. The amplitude and phase are obtained from the power and cross-power spectra of signals at positions throughout the receive aperture. The homogeneous response is estimated by averaging and is removed to isolate the aberration response. Propagation path aberration parameters calculated from pulse-echo measurements of random scattering through a tissue-mimicking aberration phantom are similar to corresponding parameters calculated for the same aberrator and array position by using echoes from a point-like reflector. The results indicate the approach is capable of describing, in addition to time-shifts, changes in waveform amplitude and shape produced by propagation through a distributed aberration

\section{0:45}

2aPA8. Simulation of ultrasonic focus aberration and correction through human tissue. Makoto Tabei (Dept. of Elec. and Computer Eng., Univ. of Rochester, Rochester, NY 14627), T. Douglas Mast (Ethicon Endo-Surgery, 4545 Creek Rd., ML 40, Cincinnati, OH 45242), and Robert C. Waag (Univ. of Rochester, Rochester, NY 14627)

Ultrasonic focusing in two dimensions has been investigated by calculating the propagation of ultrasonic pulses through cross-sectional models of the human abdominal wall and breast. Propagation calculations used a full-wave $k$-space method that accounts for spatial variations in density, sound speed, and frequency-dependent absorption and includes perfectly matched layer absorbing boundary conditions. To obtain a distorted receive wavefront, propagation from a point source through the tissue path was computed. Receive focusing used an angular spectrum method. Transmit focusing was accomplished by propagating a pressure wavefront from a virtual array through the tissue path. As well as uncompensated focusing, focusing that employed time-shift compensation and time-shift compensation after backpropagation was investigated in both transmit and receive and time reversal was investigated for transmit focusing in addition. The results indicate, consistent with measurements, that the breast causes greater focus degradation than the abdominal wall. The investigated compensation methods corrected the receive focus better than the transmit focus. Time-shift compensation after backpropagation improved the focus from that obtained using time-shift compensation alone but the improvement was less in transmit focusing than in receive focusing. Transmit focusing by time reversal resulted in lower sidelobes but larger mainlobes than the other investigated transmit focus compensation methods. 


\title{
Session 2aPPa
}

\section{Psychological and Physiological Acoustics: Hearing Aid Design: Psychophysics and Signal Processing I}

\author{
Brent W. Edwards, Cochair \\ Sound ID, 3430 West Bayshore Road, Palo Alto, California 94303 \\ Dianne J. Van Tasell, Cochair \\ Starkey Laboratories, 6600 Washington Avenue, South, Eden Prairie, Minnesota 55344 \\ Invited Papers
}

8:30

2aPPa1. State of the art in perceptual design of hearing aids. Brent W. Edwards (Sound ID, 3430 W. Bayshore Rd., Palo Alto, CA 94303, brent@edwards.net) and Dianne J. Van Tasell (Starkey Labs., Eden Prairie, MN 55344)

\begin{abstract}
Hearing aid capabilities have increased dramatically over the past six years, in large part due to the development of small, low-power digital signal processing chips suitable for hearing aid applications. As hearing aid signal processing capabilities increase, there will be new opportunities to apply perceptually based knowledge to technological development. Most hearing loss compensation techniques in today's hearing aids are based on simple estimates of audibility and loudness. As our understanding of the psychoacoustical and physiological characteristics of sensorineural hearing loss improves, the result should be improved design of hearing aids and fitting methods. The state of the art in hearing aids will be reviewed, including form factors, user requirements, and technology that improves speech intelligibility, sound quality, and functionality. General areas of auditory perception that remain unaddressed by current hearing aid technology will be discussed.
\end{abstract}

\section{$8: 45$}

2aPPa2. It's not your father's recruitment: A new view of loudness growth in cochlear hearing loss. Mary Florentine (Dept. of Speech-Lang. Path. \& Audiol. (133 FR), Northeastern Univ., 360 Huntington Ave., Boston MA 02115. florentin@ neu.edu) and Søren Buus (Comm. \& Dig. Sig. Proc. Ctr., Dept. of Elec. \& Computer Eng. (440 DA), Northeastern Univ., 360 Huntington Ave., Boston, MA 02115)

For over 60 years, one of the most commonly held beliefs in audiology has been that loudness grows more rapidly than normal near the elevated thresholds of listeners with cochlear hearing losses. This belief was based on the untested assumption that loudness at threshold is the same-often incorrectly assumed to be zero-in normal listeners and listeners with cochlear hearing losses. Recent tests of this assumption indicate that loudness at threshold is not zero in either group of listeners. These data also indicate that loudness near threshold grows at a normal rate in listeners with cochlear hearing losses. In other words, recruitment in the sense of a rapid growth of loudness near an elevated threshold does not occur. How, then, can intense sounds have approximately normal loudness in listeners with cochlear hearing losses? The explanation is that loudness at threshold is greater when the threshold is elevated by a cochlear loss than when it is normal. We call this softness imperception. Therefore, listeners with cochlear hearing losses not only have reduced dynamic range of audibility; they also have reduced dynamic range of loudness. Implications for the design of hearing aids will be discussed. [Work supported by NIH/NIDCD grant R01DC02241.]

2aPPa3. Behavioral estimates of cochlear nonlinearity and its effects on normal and impaired hearing. Andrew J. Oxenham (Res. Lab of Electron., MIT, Cambridge, MA 02139) and Christopher J. Plack (Univ. of Essex, Wivenhoe, Colchester CO4 3SQ, UK)

Recent physiological studies of basilar-membrane motion have clarified many aspects of normal and pathological cochlear processing. The compressive input-output function in response to tones around the characteristic frequency and the sharp tuning at low levels are examples of basilar-membrane properties that are thought to be important for hearing, but that are also highly vulnerable to cochlear damage. Aspects of auditory perception influenced by cochlear nonlinearity include loudness and dynamic range, temporal processing, and frequency selectivity. Functional models have assisted us in understanding the perceptual consequences of peripheral nonlinearity in a wide variety of psychoacoustic tasks. In addition, many effects of cochlear hearing loss can be simulated within such a model simply by reducing or eliminating the nonlinearity within the model. A number of behavioral measures of cochlear nonlinearity in humans have been developed in recent years. These techniques offer insights into human cochlear 
processing in general, and may also provide diagnostic information about cochlear function on an individual basis. By gaining a better understanding of the changes in cochlear processing associated with hearing loss, it may be possible to design sound-processing algorithms for hearing aids that better compensate for the effects of cochlear damage. [Work supported by NIH Grant R01DC03909.]

\section{9:45-10:00 Break}

\section{0:00}

2aPPa4. Should visual speech cues (speechreading) be considered when fitting hearing aids? Ken Grant (Walter Reed Army Medical Ctr., Army Audiol. and Speech Ctr., Washington, DC 20307-5001)

When talker and listener are face-to-face, visual speech cues become an important part of the communication environment, and yet, these cues are seldom considered when designing hearing aids. Models of auditory-visual speech recognition highlight the importance of complementary versus redundant speech information for predicting auditory-visual recognition performance. Thus, for hearing aids to work optimally when visual speech cues are present, it is important to know whether the cues provided by amplification and the cues provided by speechreading complement each other. In this talk, data will be reviewed that show nonmonotonicity between auditory-alone speech recognition and auditory-visual speech recognition, suggesting that efforts designed solely to improve auditory-alone recognition may not always result in improved auditory-visual recognition. Data will also be presented showing that one of the most important speech cues for enhancing auditory-visual speech recognition performance, voicing, is often the cue that benefits least from amplification.

\section{$10: 30$}

2aPPa5. Physiological modeling for hearing aid design. Ian C. Bruce, ${ }^{a}$ Eric D. Young, and Murray B. Sachs (Ctr. for Hearing Sci. and Dept. of Biomed. Eng., Johns Hopkins Univ., 720 Rutland Ave., Baltimore, MD 21205, ibruce@ieee.org)

Physiological data from hearing-impaired cats suggest that conventional hearing aid signal-processing schemes do not restore normal auditory-nerve responses to a vowel [Miller et al., J. Acoust. Soc. Am. 101, 3602 (1997)] and can even produce anomalous and potentially confounding patterns of activity [Schilling et al., Hear. Res. 117, 57 (1998)]. These deficits in the neural representation may account at least partially for poor speech perception in some hearing aid users. An amplification scheme has been developed that produces neural responses to a vowel more like those seen in normal cats and that reduces confounding responses [Miller et al., J. Acoust. Soc. Am. 106, 2693 (1999)]. A physiologically accurate model of the normal and impaired auditory periphery would provide simpler and quicker testing of such potential hearing aid designs. Details of such a model, based on that of Zhang et al. [J. Acoust. Soc. Am. 109, 648 (2001)], will be presented. Model predictions suggest that impairment of both outer- and inner-hair cells contribute to the degraded representation of vowels in hearing-impaired cats. The model is currently being used to develop and test a generalization of the Miller et al. speech-processing algorithm described above to running speech. [Work supported by NIDCD Grants DC00109 and DC00023.] a) Now with the Dept. of Electrical and Computer Engineering, McMaster Univ., 1280 Main St. W., Hamilton, ON L8S 4K1, Canada.

\section{1:00}

2aPPa6. Biologically inspired binaural hearing aid algorithms: Design principles and effectiveness. Albert Feng (Beckman Inst., Univ. of Illinois, Urbana, IL 61801)

Despite rapid advances in the sophistication of hearing aid technology and microelectronics, listening in noise remains problematic for people with hearing impairment. To solve this problem two algorithms were designed for use in binaural hearing aid systems. The signal processing strategies are based on principles in auditory physiology and psychophysics: (a) the location/extraction (L/E) binaural computational scheme determines the directions of source locations and cancels noise by applying a simple subtraction method over every frequency band; and (b) the frequency-domain minimum-variance (FMV) scheme extracts a target sound from a known direction amidst multiple interfering sound sources. Both algorithms were evaluated using standard metrics such as signal-tonoise-ratio gain and articulation index. Results were compared with those from conventional adaptive beam-forming algorithms. In free-field tests with multiple interfering sound sources our algorithms performed better than conventional algorithms. Preliminary intelligibility and speech reception results in multitalker environments showed gains for every listener with normal or impaired hearing when the signals were processed in real time with the FMV binaural hearing aid algorithm. [Work supported by NIH-NIDCD Grant No. R21DC04840 and the Beckman Institute.] 


\title{
Session 2aPPb
}

\section{Psychological and Physiological Acoustics: Potpourri (Poster Session)}

\author{
John G. Neuhoff, Chair \\ Psychology Department, College of Wooster, Wooster, Ohio 44691
}

\section{Contributed Papers}

\begin{abstract}
All posters will be on display from 8:30 a.m. to 5:00 p.m. To allow contributors an opportunity to see other posters, contributors of odd-numbered papers will be at their posters from 8:30 a.m. to 10:15 a.m. and contributors of even-numbered papers will be at their posters from 10:15 a.m. to 12 noon. To allow for extended viewing time, posters will remain on display until 5:00 p.m.
\end{abstract}

\begin{abstract}
2aPPb1. A cortical network underpinning the perceptual priority for rising intensity and auditory "looming." John G. Neuhoff (Dept. of Psych., The College of Wooster, Wooster, OH 44691, jneuhoff@wooster.edu), Deniz Bilecen, Henrietta Mustovic, Hartmut Schachinger, Erich Seifritz (Univ. of Basel, Basel, Switzerland), Klaus Scheffler (Univ. of Freiburg, Freiburg, Germany), and Francesco Di Salle (Univ. of Naples Federico II, Naples, Italy)
\end{abstract}

Relative motion between a sound source and a listener creates a change in acoustic intensity that can be used to anticipate the source's approach. Humans have been shown to overestimate the intensity change of rising compared to falling intensity sounds and underestimate the timeto-contact of approaching sound sources. From an evolutionary perspective, this perceptual priority for looming sounds may represent an adaptive advantage that provides an increased margin of safety for responding to approaching auditory objects. Here, using functional magnetic resonance imaging, we show that the prioritization of rising contrasted with falling intensity sine-tones is grounded in a specific neural network. This network is predominantly composed of the superior temporal sulci, the middle temporal gyri, the right temporo-parietal junction, the motor and premotor cortices mainly on the right hemisphere, the left frontal operculum, and the left superior posterior cerebellar cortex. These regions are critical for the allocation of attention, the analysis of space, object recognition, and neurobehavioral preparation for action. Our results identify a widespread neural network underpinning the perceptual priority for looming sounds that can be used in translating sensory information into preparedness for adverse events and appropriate action. [Work supported by the Swiss and the American NSFs.]

2aPPb2. Effects of spatially correlated acoustic-tactile information on judgments of auditory circular direction. Annabel J. Cohen, M. J. Reina Lamothe, Ian D. Toms, and Richard A. G. Fleming (Dept. of Psych., Univ. of PE, Charlottetown, PE C1A 4P3, Canada)

Cohen, Lamothe, Fleming, MacIsaac, and Lamoureux [J. Acoust. Soc. Am. 109, 2460 (2001)] reported that proximity governed circular direction judgments (clockwise/counterclockwise) of two successive tones emanating from all pairs of 12 speakers located at 30-degree intervals around a listeners' head (cranium). Many listeners appeared to experience systematic front-back confusion. Diametrically opposed locations (180degrees - theoretically ambiguous direction) produced a direction bias pattern resembling Deutsch's tritone paradox [Deutsch, Kuyper, and Fisher, Music Percept. 5, 7992 (1987)]. In Experiment 1 of the present study, the circular direction task was conducted in the tactile domain using 12 circumcranial points of vibration. For all 5 participants, proximity governed direction (without front-back confusion) and a simple clockwise bias was shown for 180-degree pairs. Experiment 2 tested 9 new participants in one unimodal auditory condition and two bimodal auditory-tactile conditions (spatially-correlated/spatially-uncorrelated). Correlated auditory-tactile information eliminated front-back confusion for 8 participants and replaced the "paradoxical" bias for 180-degree pairs with the clockwise bias. Thus, spatially correlated audio-tactile location information improves the veridical representation of 360-degree acoustic space, and modality-specific principles are implicated by the unique circular direction bias patterns for 180-degree pairs in the separate auditory and tactile modalities. [Work supported by NSERC.]

2aPPb3. Localization judgments are sensitive to late-arriving sound. G. Christopher Stecker (Kresge Hearing Res. Inst., Univ. of Michigan, 1301 E. Ann St., Ann Arbor, MI 48109-0519, cstecker@umich.edu) and Ervin R. Hafter (Univ. of California at Berkeley, Berkeley, CA 94720-1650)

In a study of temporal aspects of sound localization, free-field listeners localized trains of 2-32 spatially distributed clicks (4-kHz narrow-band impulses) in a pointing task. Multiple regression coefficients estimated the perceptual "weight" listeners applied to each click in a train when making location judgments. Temporal weighting functions (TWFs) obtained in this way exhibited two dominant features: First, at high stimulus rates $(>80 \mathrm{~Hz})$, weight for the first click in a train was significantly larger than that for later clicks, as observed previously in studies measuring TWFs for ITD-based lateralization [K. Saberi, Percept. Psychophys. 58, 1037-1046 (1998); R. Dizon et al., Assoc. Res. Otolaryngol. Abs. 21, 42 (1998)]. Second, in several conditions, clicks near the end of a train received larger weights than earlier clicks. This presentation focuses on the latter finding, which affected the last few clicks in a train despite manipulations of train length (overall duration) and predictability, and which appeared independently of increased weight on the first click. The effect cannot be explained as a release from temporary suppression, but may relate to effects of temporal integration, apparent motion, or the influence of non-ITD spatial cues. [Work supported by NIDCD 00087.]

2aPPb4. Simple neuron models of ITD sensitive neurons. Vasant Dasika (Hearing Res. Ctr. and Dept. of Biomed. Eng., Boston Univ., Boston, MA 02215), John A. White, and H. Steven Colburn (Boston Univ., Boston, MA)

Neurons which show sensitivity to interaural time delay (ITD) exist in both mammalian medial superior olive (MSO), and bird nucleus laminaris (NL). In this study, we examine simple mathematical models of single MSO and NL cells which respond probabilistically to a pair of isolated inputs with a response probability that depends on the input interpulse interval. Inputs are either isolated pulse pairs or pairs of periodic trains, with or without random jitter added to their event times. Refractoriness is incorporated in the input description and/or in the cell model in specified simulations. We find that periodic rate-ITD shapes are shaped by three 
interacting factors: the cell's temporal response (described by the pairedpulse response), input frequency, and the degree of input synchrony. Paired-pulse responses are able to predict the widths of rate-ITD curves obtained from deterministic periodic input simulations. Reduced input synchrony predictably smears rate-ITD curves. Larger numbers of weaker inputs yield stronger rate-ITD modulation than a few strong inputs. Model response is compared with in vivo and in vitro MSO and NL physiological data. Comparisons with published analytical models as well as more complex and realistic physiological cell models are examined.

2aPPb5. JNDS of interaural time delay (ITD) of selected frequency bands in speech and music signals. Avner Aliphas, H. Steven Colburn (Hearing Res. Ctr. and Dept. of Biomed. Eng., Boston Univ., Boston, MA 02215), and Oded Ghitza (Agere Systems)

JNDS of interaural time delay (ITD) of selected frequency bands in the presence of other frequency bands have been reported for noiseband stimuli [Zurek (1985); Trahiotis and Bernstein (1990)]. Similar measurements will be reported for speech and music signals. When stimuli are synthesized with bandpass/band-stop operations, performance with complex stimuli are similar to noisebands (JNDS in tens or hundreds of microseconds); however, the resulting waveforms, when viewed through a model of the auditory periphery, show distortions (irregularities in phase and level) at the boundaries of the target band of frequencies. An alternate synthesis method based upon group-delay filtering operations does not show these distortions and is being used for the current measurements. Preliminary measurements indicate that when music stimuli are created using the new techniques, JNDS of ITDs are increased significantly compared to previous studies, with values on the order of milliseconds.

2aPPb6. Trajectory perception in the free field. Joshua A. Miele and Ervin R. Hafter (Dept. of Psych., Univ. of California, Berkeley, CA 94720, jam@ @ocrates.berkeley.edu)

Using virtual sound sources in an echo-attenuated chamber, the sensitivity of subjects to the direction of linear auditory motion was evaluated. Four sighted and four blind subjects participated. Subjects reported the perceived trajectory by orienting a freely rotating pointer. Subjects fell into two distinct categories: those who performed well at the task, and those who did not, with blind subjects tending to be in the first category and sighted subjects the second. Those subjects performing well at the task also showed improved performance with increasing linear velocity. Because the only stimulus parameter associated with increased linear velocity was frequency envelope, it can be concluded that frequency changes resulting from the Doppler effect can improve the accuracy of auditory trajectory perception. The same subjects performed discrimination tasks measuring sensitivity to changes in azimuthal acceleration, as well as to changes in amplitude and frequency envelopes. Two models of trajectory perception are considered: A Psychoacoustical Parameters Model uses a weighted combination of specific perceptual abilities such as sensitivity to azimuthal acceleration, and to changes in amplitude and frequency envelopes. An Informational Masking Model is considered as a possible explanation for those subjects demonstrating poor performance in the trajectory perception task.

2aPPb7. Localization of noise bursts in the median plane: Effects of duration and level. Gongqiang Yu, Ruth Y. Litovsky, and Frederic L. Wightman (Waisman Ctr., Univ. of Wisconsin-Madison, Madison, WI 53705, yu@waisman.wisc.edu)

Previous research has revealed that human sound localization in the median sagittal and in the front/back dimension may depend on the listener's ability to reliably extract information regarding characteristic peaks and notches of the source spectrum upon reaching the ears. While longduration sounds are well localized using spectral cues, the processing of spectral cues for sounds of short duration appears to be degraded. The mechanisms involved in "image formation" in the localization process, and the relative importance of sound duration and level are not well understood. In the present study, the effect of signal duration and level on localization performance, with an emphasis on the median-sagittal plane were systematically explored. Three types of stimuli, clicks, noises, and trains of frozen and random noise bursts encompassing the same time period as the noises, with different duration and at various levels, were randomly presented to the loudspeakers. Subjects were blindfolded, but were instructed to move their heads as necessary, to maximize their confidence regarding source position judgment. Results will be discussed in relation to possible mechanisms employed by the auditory system to utilize spectrally shaped cues in the localization process. [Work supported by NIDCD Grant Nos. R29 DC03083 and P01 DC 00116.]

2aPPb8. Spectral-temporal analysis of OAEs in normal-hearing humans: Latencies, fine structure, and multiple internal reflections. Dawn Konrad-Martin (Dept. of Commun. Disord. and Sci., Rush Univ., 1653 W. Congress Pkwy., Rm. 1015 AAC, Chicago, IL 60612), Douglas H. Keefe, and Jeffrey L. Simmons (Boys Town Natl. Res. Hospital, Omaha, NE 68131)

Otoacoustic emissions (OAEs) display complexity in both time and frequency, which may be obscured in traditional response analyses. Timefrequency representations (TFR) of the eliciting stimuli and the recorded OAE provide a tool to understand this complexity. TFRs were applied to stimulus-frequency (SF) and distortion-product (DP) OAEs obtained in 25 normal-hearing adults, using three classes of stimuli: continuous tones, gated tones (tonal stimuli with well-defined onset, steady state and decay), and tone pips (band-limited impulses). Synchronous spontaneous OAEs were measured using clicks to assess their contributions to TFRs of SFOAE and DPOAEs. A common form of TFR generated from tone-pips is a collection of frequency-specific components, with each component characterized by one or more brief segments or a single long-duration segment. Frequency-pulling effects between components were observed, however, little evidence was found for interactions within transient SFOAEs in the form of intermodulation distortion. TFRs show evidence for strong emission regions or cochlear "hot spots" within pip responses, and multiple internal reflections within gated and pip responses. The resulting measures of OAE latencies are compared with model predictions. Thus, the TFR analysis provides a quantitative tool for measuring the spectral-temporal organization of multiple-component OAEs. [Work supported by NIH, DC003784.]

2aPPb9. SFOAE input/output functions elicited by slightly off-frequency suppressors. Kim S. Schairer and Douglas H. Keefe (Boys Town Natl. Res. Hospital, 555 N. 30th St., Omaha, NE 68131, schairerk@boystown.org)

Basilar membrane (BM) response growth is thought to be related to stimulus frequency otoacoustic emission (SFOAE) input/output (I/O) functions. SFOAEs comprise the response to sinusoidal excitation and provide an estimate of nonlinearity at a tonotopic place on the BM. SFOAE I/O functions at frequency $f_{0}$ (across levels $L_{0}$ ) are measured in the presence of suppressor $f_{s}$ (at level $L_{s}$ ) in subjects with normal hearing. $f_{s}$ is varied below, equal to, and above $f_{0}$ in the range $0.93<f_{s} / f_{0}$ $<1.07$. For fixed suppressor level $\left(L_{s}=80 \mathrm{~dB}\right.$ SPL) and varying $L_{0}$ $<L_{s}$, the SFOAE I/O function is predicted to be closely related to the BM $\mathrm{I} / \mathrm{O}$ function. Measured I/O functions are approximately invariant as $f_{s}$ is varied. Functions saturate at increasing levels with a decrease in some ears at the highest levels. An alternative paradigm in which $L_{s}=L_{0}$ are covaried yields SFOAE I/O functions with local minima, but which may have a less direct connection to $\mathrm{BM}$ response growth. Based on primaries at $f_{s}$ and $f_{0}$, SFOAE and DPOAE I/O functions differ. One implication is that SFOAE I/O functions may be used to relate BM nonlinear response growth to perceptual processes, including loudness growth and forward masking. [Work supported by NIH (R01 DC003784, T32 DC00013).] 
2aPPb10. The effect of ear canal pressure on pure-tone thresholds and middle-ear reflectance and conductance. M. Patrick Feeney, Julie Hazelbaker, J. Kip Kelly, and Amanda Graves (Speech and Hearing Sci., Ohio State Univ., Columbus, OH 43210)

The effect of ear canal pressure on pure-tone thresholds and wideband reflectance and conductance was examined in ten human participants. Pure-tone thresholds at $0.5,1,2$, and $4 \mathrm{kHz}$ were measured in random order using a $2 \mathrm{AFC}$ procedure at ambient pressure, and at ear canal pressures of positive and negative $200 \mathrm{daPa}$. These results were compared to wideband changes in reflectance and conductance using the same probe. Reflectance changes with pressure were in good agreement with recent data on reflectance tympanometry [R. H. Margolis, G. L. Saly, and D. H. Keefe, J. Acoust. Soc. Am. 106, 265-280 (1999)]. Both one-third-octave conductance measures and pure-tone thresholds were found to decrease by around $10 \mathrm{~dB}$ at $500 \mathrm{~Hz}$ and $1000 \mathrm{~Hz}$ compared to a 2- to 4- $\mathrm{dB}$ decrease at 2000 and $4000 \mathrm{~Hz}$. These results are consistent with the change in middle-ear sound transmission with ear-canal pressure changes reported in a recent temporal-bone study in Norwegian cattle [M. Kringlebotn, J. Acoust. Soc. Am. 107, 1442-1450 (2000)]. The results of this study suggest that the change in middle-ear conductance may provide an objective measure of conductive hearing loss for the conditions in this experiment. [Work supported by NIH, RO3 DC04129.]

2aPPb11. Vibrotactile suppression of tinnitus. Martin L. Lenhardt (Prog. in Biomed. Eng. and Dept. of Otolaryngol., Virginia Commonwealth Univ., Richmond, VA 23298-0168)

At the Society's 142nd meeting, the efficacy of high frequency bone conducted stimulation in suppressing tinnitus was presented. The hypothesized mechanism was the reprogramming of frequency tuning of auditory neurons in the central nervous system, secondarily to peripheral hearing loss. This mechanism is unlikely in cases of tinnitus in the presence of normal audiometric sensitivity. There is the possibility that hearing loss above $10 \mathrm{kHz}$ can play a role in tinnitus, an association not thoroughly explored. Somatomotor stimulation influencing the quality of tinnitus has been reported, as have interconnections of the auditory and somatosensory systems. There would appear to be an evolutionary advantage of linking the sensorimotor organization of the external ear and the auditory function of the brainstem in sound localization. Thus, stimulation of the pinna and post auricular area may be a means of suppressing tinnitus. To that end a thin aluminum ceramic bimorph was constructed to fit on the inner surface of the pinna. When driven by low $(<100 \mathrm{~Hz})$ and high $(>10 \mathrm{kHz})$ frequencies multiplied by $\mathrm{MHz}$ carriers, demodulation in the skin resulted in vibrotactile stimulation. Tactile stimulation was an adjunct to the high frequencies resulting in a multimodal suppressive effect in a small pilot study.

2aPPb12. On the perceptual and behavioral significance of saccular acoustic sensitivity. Neil P. M. Todd (Dept. of Psych., Univ. of Manchester, Manchester M13 9PL, UK)

Evidence accumulated over the last few decades supports the case that the sacculus has conserved an acoustic sensitivity throughout vertebrate phylogeny [N. P. Todd, J. Acoust. Soc. Am. 110, 380-390 (2001)]. In humans saccular acoustic sensitivity may be demonstrated by means of myogenic vestibular evoked potentials (MVEP) but the question remains whether saccular acoustic sensitivity in humans has any perceptual or behavioral significance. It has been shown that MVEP have the following properties: (1) MVEP can be obtained to natural acoustic stimuli above about $90 \mathrm{~dB}$ SPL; (2) MVEP have a frequency tuning property with a best frequency between $300 \mathrm{~Hz}-350 \mathrm{~Hz}$; (3) MVEP exhibit adaptation, characteristic or normal sensory processing; and (4) there is a change of the quality of sensation above the MVEP threshold, indicative that saccular acoustic sensitivity may play a role in the perception of loud sound. In this paper new evidence is discussed which indicates that both myogenic and neurogenic vestibular evoked potentials (NVEP), particularly those obtainable from prefrontal cortex, may be linked to subjective and behavioral responses to loud sound. These responses may in turn be related to individual differences in the functioning of the mesolimbic dopamine system, including prefrontal cortex.

2aPPb13. A chinchilla nonlinear cochlear filterbank. Alberto LopezNajera, Enrique A. Lopez-Poveda (Centro Regional de Investigación Biomédica, Facultad de Medicina, Universidad de Castilla, La Mancha, 02071 Albacete, Spain), and Ray Meddis (Univ. of Essex, Wivenhoe Park, Colchester CO4 3SQ, UK)

A dual-resonance nonlinear (DRNL) filter [Meddis et al., J. Acoust. Soc. Am. 106, 2852-2861 (2001)] was fitted to model chinchilla cochlear responses to tonal stimuli at individual sites along the basilar membrane (BM) with best frequencies (BF) of $0.8,5.5,7.25,9.75,10.0,12.0$, and $14.0 \mathrm{kHz}$. At each BF, parameters were obtained for the DRNL filter to reproduce input/output and tuning curves. The match between the model and the experimental data is almost perfect for frequencies near BF. Quantitatively, the model response gets worse (but is still reasonable) for frequencies well below and well above BF. These discrepancies are discussed in terms of the middle-ear function, which proves critical. The model responses to clicks, AM, multicomponent, and Schroder-phase stimuli were also compared against experimental data. Results show that the architecture of the DRNL filter seems suitable to reproduce this wide range of phenomena. Strategies are discussed for developing a chinchilla nonlinear cochlear filterbank from current parameters. [Work supported by the Consejería de Sanidad of the Junta de Comunidades of Castilla, La Mancha.]

2aPPb14. Estimation of size at three sites on the face. Ronald $\mathrm{T}$. Verrillo (Inst. for Sensory Res., Syracuse Univ., Syracuse, NY 13244), Stanley J. Bolanowski (Inst. for Sensory Res./Dept. of Bioengineering and Neurosci., Syracuse Univ., Syracuse, NY 13244), and Francis P. McGlone (Unilever Res., Bebington, Wirral L63 3J2, UK)

The subjective size of steel balls was judged by nine subjects using the method of Absolute Magnitude Estimation. The balls were rolled ("scripted") over three areas of the face (forehead, cheek, and lower lip) using the right index distal finger pad. The areas were stimulated under three conditions; (1) the subjects upon themselves (intra-active touch); (2) the subjects upon another person (interactive touch); and (3) another person upon the face of the subject (interactive touch). There were significant differences among the three facial sites in the intra-active condition. When another person's face was scripted by the subject, there were no differences among the sites. The results were mixed when another scripted upon the faces of the subjects. The results are considered in terms of tissue mechanics, mode of stimulation and representation of the sites on the somatosensory cortex. [Work supported by NIH.]

2aPPb15. Underwater loudness for tones. Edward A. Cudahy and Derek Schwaller (Naval Submarine Medical Res. Lab., Groton, CT 06349-5900)

The loudness for pure tones was measured by loudness matching for 1-s pure tones from 100 to $50000 \mathrm{~Hz}$. The standard tone was $1000 \mathrm{~Hz}$. Subjects were instructed to match the loudness of the comparison tone at one of the test frequencies to the loudness of the standard tone. The standard was presented at one of five sound pressure levels (SPL) for each set of frequencies. The SPL was varied randomly across a test series. The subjects were bareheaded U.S. Navy divers tested at a depth of $3 \mathrm{~m}$. All subjects had normal hearing. The tones were presented to the right side of the subject from an array of underwater sound projectors. The SPL was calibrated at the location of the subject's head with the subject absent. The loudness increased more rapidly as a function of standard SPL at midfrequencies than at either high or low frequencies. The most compact loudness contours (the least SPL change across the range of standard SPL) were at the highest frequency. Loudness contours across frequency derived 
from these measurements are significantly different from in-air measurements with minimum audibility in the $1000 \mathrm{~Hz}$ region rather than the 2-4 $\mathrm{kHz}$ region observed for in-air measurements.

2aPPb16. Sample discrimination of frequency differences with irrelevant context-revisited. Donna L. Neff, Eric C. Odgaard, and Walt Jesteadt (Boys Town Natl. Res. Hospital, 555 N. 30th St., Omaha, NE 68131)

This experiment replicated earlier work to provide additional data for post hoc analyses of perceptual weights for conditions with large effects of context on performance. The non-adaptive, 2AFC task was sample discrimination of frequency differences (SD-F) in quiet and with added context stimuli. Listeners were to select the interval in which a pair of target tones was drawn from the higher of two Gaussian frequency distributions (means of 2000 and $2150 \mathrm{~Hz}$ ). Pairs of context stimuli were added at frequency regions above and below the targets, at distances of 600, 1000 and $1400 \mathrm{~Hz}$. Context stimuli were fixed-frequency tones, noise bands, or random-frequency tones. All stimuli were $100 \mathrm{~ms}$ with 5 -ms ramps, presented simultaneously. Conditions were tested with and without Gaussian level jitter (standard deviation $=3 \mathrm{~dB}$ ). Mean levels of target and context stimuli were equated or systematically varied across conditions. The results confirm earlier work, with little effect of fixed-frequency tones or noise-band context at any distance from the targets. Random-frequency context tones produced large detrimental effects even at remote distances, due predominantly to effects of the lower-frequency context tone. Level variation had little effect. Different approaches to calculating perceptual weights influences data interpretation. [Work supported by NIDCD.]

2aPPb17. Estimating the form of the peripheral nonlinearity from slopes of psychometric functions in forward masking. Walt Jesteadt, Stephen T. Neely, and Kim S. Schairer (Boys Town Natl. Res. Hospital, 555 N. 30th St., Omaha, NE 68131, jesteadt@ boystown.org)

In previous work, we have shown that slopes of psychometric functions (PF) obtained for the detection of a signal in the presence of a forward masker are in general agreement with standard assumptions concerning the form of the peripheral nonlinearity. PFs are steep for conditions where signal levels are low and grow progressively shallower as the signal threshold increases in level. PFs become more parallel if signal levels are adjusted to reflect the effect of a nonlinearity of the form proposed by Yates et al. [G. K. Yates, I. M. Winter, and D. Robertson, Hear. Res. 45, 203-220 (1990)]. An alternative approach is to use PF data to estimate the form of the nonlinearity. The data suggest that the reciprocal of the slope of the PF is linearly related to the signal level. This would indicate that the nonlinearity is logarithmic. Use of a logarithmic nonlinearity as the first stage in a model of forward masking predicts thresholds as a function of the masker level and signal delay that are consistent with a formula proposed by Jesteadt et al. [W. Jesteadt, S. P. Bacon, and J. R. Lehman, J. Acoust. Soc. Am. 71, 950-962 (1982)] and predicts loudness growth rates consistent with the literature. [Work supported by NIDCD.]

2aPPb18. Filter asymmetry for short-duration signals as a function of signal delay from masker onset. Veronica Pimentel and Elizabeth A. Strickland (Dept. of Audiol. and Speech Sci., Purdue Univ., West Lafayette, IN 47907, estrick@purdue.edu)

In a previous study, filter shapes were measured for short-duration tones at the onset or in the center of a longer-duration, notched-noise masker. The filter shapes sharpened with signal delay, with the amount of sharpening depending on the level of the signal. The pattern of results was interpreted in terms of a model incorporating published physiological data on nonlinear basilar-membrane input-output functions. The results suggested that these functions might become more linear as the signal is delayed from masker onset. The present study extends this approach to asymmetric notches. This was motivated by previous research suggesting that frequencies above the signal frequency might produce more of a temporal effect than those below the signal frequency, and by physiological data showing that basilar-membrane input-output functions differ for tones above and below the characteristic frequency. The signal was a 10-ms, 4-kHz sinusoid, fixed at levels from 40 to $70 \mathrm{~dB}$ SPL, and presented at the onset or in the center of a notched-noise masker. Notches were placed symmetrically or asymmetrically about the signal. The results will be discussed in terms of basilar-membrane input-output functions.

2aPPb19. Similar improvements in auditory discrimination produced by multiple-hour training with a labeling or discrimination procedure. Julia A. Mossbridge and Beverly Wright (Dept. of Commun. Sci. and Disord. and Northwestern Univ. Inst. for Neurosci., $2299 \mathrm{~N}$. Campus Dr., Northwestern Univ., Evanston, IL 60208)

Does the procedure used to train an auditory skill impact the characteristics of subsequent skill improvement? Here, the effects of multiplehour training with either a labeling or discrimination procedure were compared by using a discrimination procedure to examine performance on six conditions. Listeners completed a pretest on the six conditions, trained on one condition for 720 trials/day for 8 days with either a labeling $(n=6)$ or discrimination $(n=6)$ procedure, then completed a post-test identical to the pretest. The trained condition for labeling-trained listeners was to determine, for each individual presentation, whether the onsets of two tones were synchronous or asynchronous. The trained condition for discrimination-trained listeners was to determine which of two presentations of these same tones had asynchronous onsets. All listeners showed significant improvements on the trained condition. Further, there were no significant differences between training groups in the pattern of improvement across the six conditions, both immediately and one month after training. Thus, multiple-hour labeling and discrimination training yielded similar learning, suggesting that, in this case, both procedures tapped the same neural substrate. These results inform our understanding of the mechanisms of learning and may guide clinical treatment for those with auditory perceptual disorders. [Work supported by NIDCD.]

2aPPb20. Cut-back frozen noise characterization of auditory sensory memory. Anthony Boemio and David Poeppel (Cognit. Neurosci. of Lang. Lab., 3416 Marie Mount Hall, Univ. of Maryland, College Park, MD 20742, aboemio@glue.umd.edu)

Frozen noise stimuli were comprised of repeated concatenated segments of random noise, with the segment duration determining the periodicity or stimulus onset asynchrony (SOA). Seven conditions were constructed with SOAs ranging from 100-1000 ms. For each of the seven conditions, a fraction of the repeated segments were "cut-back" with nonrepeated noise. Subjects were presented with either a frozen noise stimulus or a nonrepeated noise stimulus of equal duration, and told to indicate if they detected periodicity. The thresholds for this two-alternative forcedchoice infrapitch periodicity detection task were obtained by an adaptive tracking procedure. The results show that classification performance varies linearly with SOA over the entire 100-1000 ms. range. When normalized by SOA, it becomes evident that detection is characterized by a signal-tonoise ratio (SNR) where the repeated noise is the signal and the nonrepeated noise, the noise. The SNR for all seven conditions was approximately 0.7. It is proposed that cut-back frozen noise stimuli may be used to establish the upper storage limit of auditory sensory memory by extending this technique to SOAs greater than $1000 \mathrm{~ms}$ and looking for deviation from linearity in the plot of repeated noise segment vs SOA. [Work supported by NIDCD Grant No. DC 0463801.] 
2aPPb21. Perception of duration of ramped and damped sounds with exponential and raised cosine ramps. Massimo Grassi (Dept. of Gen. Psych., Univ. of Padova, via Venezia 8, 35131 Padova, Italy, grassi@psy.unipd.it) and Christopher J. Darwin (Univ. of Sussex, Brighton BN1 9QG, UK)

In the first experiment listeners were asked to adjust the duration of a steady-level sound in order to match the duration of 250-, 500-, 750-, or 1000-ms targets. Targets could be either steady in level, increasing in level with either a raised-cosine or an exponential ramp (ramped sounds), or the ramped sounds reversed in time (damped sounds). The results extended to longer durations than those reported by Schlauch et al. [J. Acoust. Soc.
Am. 109, 2880-2887 (2001)]: damped sounds were matched as much shorter ( $\sim 35 \%$ for exponential ramps, $\sim 30 \%$ for raised-cosine ramps) and ramped sounds as slightly shorter $(\sim 10 \%)$ than steady-level sounds. In the second experiment, the subjective duration of all targets used in the first experiment was measured as the difference between reaction times to their onsets and their offsets. Although delays in reaction times to onsets of ramped targets could explain their slight underestimation, anticipations to offsets of damped targets were too small to explain their underestimation. The results of the research contradict the predictions of the AIM model [R. D. Patterson and T. Irino, J. Acoust. Soc. Am. 104, 2967-2979 (1998)], where ramped and damped sounds longer than $200 \mathrm{~ms}$ should be perceived as possessing similar subjective durations.

\title{
Session 2aSA
}

\section{Structural Acoustics and Vibration: Pyroshock I}

\author{
Harry Himelblau, Chair \\ Space and Communication Division, The Boeing Company, Rocketdyne Propulsion and Power, \\ Canoga Park, California 91309-7922
}

\section{Invited Papers}

\section{$8: 45$}

2aSA1. Pyrotechnic devices and their applications. Harry Himelblau (MC IB-31, Rocketdyne Propulsion and Power, The Boeing Co., Canoga Park, CA 91309-7922, harry.himelblau@boeing.com)

Pyroshock is mechanical shock transmitted through structures from explosive devices, sometimes accompanied by structural impact. These devices are designed to cause the intentional separation of structures, or to cause the deployment of various mechanisms or subsystems required for mission operation. Separation devices usually fall into two categories: (a) line sources, such as linear shaped charges, and (b) point sources, such as explosive bolts, pin puller and pushers, and gas generators. The advantages of these devices are high reliability (especially when redundantly activated), low cost and weight, high activation speed, and low structural deformation a short distance from the source. The major limitation is pyroshock, a severe high-frequency transient capable of causing failure or malfunction to small nearby elements, especially electronic and optical components located close to the source. This pyroshock tutorial, which is intended to summarize recent improvements to the technology, is initiated with a review of explosive and companion devices.

9:15

2aSA2. Pyroshock prediction procedures. Allan G. Piersol (Piersol Eng. Co., 23021 Brenford St., Woodland Hills, CA 91364-4830, apiersol@pacbell.net)

Given sufficient effort, pyroshock loads can be predicted by direct analytical procedures using Hydrocodes that analytically model the details of the pyrotechnic explosion and its interaction with adjacent structures, including nonlinear effects. However, it is more common to predict pyroshock environments using empirical procedures based upon extensive studies of past pyroshock data. Various empirical pyroshock prediction procedures are discussed, including those developed by the Jet Propulsion Laboratory, LockheedMartin, and Boeing.

\section{$9: 45$}

2aSA3. Pyroshock data acquisition—historical developments using piezoelectric accelerometers and other transducers. Harry Himelblau (MC IB-31, Rocketdyne Propulsion and Power, The Boeing Co., Canoga Park, CA 91309-7922, harry.himelblau@boeing.com)

For nearly 50 years, $\mathrm{P} / \mathrm{E}$ accelerometers have been used for acquiring pyroshock data with mixed results. For longer distances between the explosive source and the transducer location (e.g., two feet or more), valid data of lesser shock magnitude were usually obtained. However, for shorter distances, a variety of problems were often encountered, causing erroneous results. It was subsequentially determined that most problems were caused by measurement system nonlinearities, i.e., the nonlinear resonant response of the 
accelerometer, or exceeding the linear amplitude range of the signal conditioner and recorder. In the earlier years, it was erroneously assumed that subsequent low pass filtering of the signal would remove the nonlinearities, hopefully leading to valid data. This only masked the invalid results. Eventually, improved P/E accelerometers were developed with higher natural frequencies and larger amplitude limits that caused substantially fewer problems and allowed measurements closer to the explosive sources. Shortly thereafter, the high frequency noncontact laser doppler vibrometer became available which circumvented the accelerometer resonance problem. However, this velocity transducer is almost always limited to laboratory tests in order to constrain the motion of the laser head by a very rigid and massive support foundation compared to the flexible structure which is attached to the laser target. Other LDV measurement problems have been encountered that must be avoided to achieve valid data. Conventional strain gages have been successfully used to measure pyroshock strain. However, due to the short wavelength of direct and bending pyroshock waves at high frequencies, small strain gages are usually required to avoid spatial averaging over the length of the gage.

10:15-10:30 Break

10:30

2aSA4. Pyroshock data acquisition—recent developments using $\mathbf{P} / \mathbf{R}$ and $\mathbf{P} / \mathbf{E}$ accelerometers and isolators. Vesta I. Bateman (Sandia Natl. Labs., P.O. Box 5800, Albuquerque, NM 87185-0553, vibatem@sandia.gov)

Mechanical isolators have been developed for piezoresistive and piezoelectric accelerometers to mitigate high frequency shocks before they reach the accelerometer because the high frequency pyroshocks may cause the accelerometer to resonate and/or break. Several commercial mechanically isolated accelerometers are available to the general public and their characteristics have been studied using Hopkinson bar test techniques. The in-axis response of these devices will be compared. Cross-axis response will be presented for one device. Additionally, pyroshock and ballistic shock measurements, performed by international organizations, will be presented for several isolators.

11:00

2aSA5. Pyroshock data analysis—Analysis, editing, and validation. David O. Smallwood (Sandia National Labs., P.O. Box 5800, Albuquerque, NM 87185-0553, dosmall@sandia.gov)

The most common form of pyroshock data analysis is the shock response spectrum (SRS), which is defined and briefly discussed. The most common way to compute the SRS of a time history is with a digital recursive filter that simulates the response of a single-degree-of-freedom (SDOF) system to the pyroshock excitation. This filter is described together with its strengths and limitations. Sometimes additional characteristics of the time history are used to supplement the SRS. The most common is a definition of duration. If the time history is assumed to be a sum of exponentially decaying sinusoids, a decay rate can be used. Often, the time duration is defined as the duration until the waveform decays to $10 \%$ of its peak value. More recently temporal moments have been used to define the temporal characteristics of the waveforms. Flaws in pyroshock data are common, including accelerometer zero shifts, clipped data, dropouts, and instrumentation overloads. Data validation procedures that expose many of these flaws are outlined. Good pyroshock data are difficult to acquire. As a general rule, flawed data should not be used, but sometimes we are forced to use flawed data. Procedures are briefly described for editing flawed data that will result in credible, although not necessarily correct, results.

11:30

2aSA6. Pyroshock data analysis-The GCPP validation procedure. Allan G. Piersol (Piersol Eng. Co., 23021 Brenford St., Woodland Hills, CA 91364-4830, apiersol@ pacbell.net)

This procedure was developed for validating pyroshock data by Powers and the author, using techniques originated by Gaberson and Chalmers in validating naval shock data at lower frequencies and subsequently modified. It requires that the acceleration time history be single and double integrated to obtain a velocity and a displacement time history, which is to be examined. Valid data look like a low-pass filtered acceleration time history which is not integrated. The maximum resulting displacement should approximate the independently measured displacement (often near zero) or that previously computed analytically for the structure at the measured location. In addition, the positive and negative SRS are computed and compared. Valid data usually show similar spectral content. 


\title{
Session 2aSC
}

\section{Speech Communication: Cross-Linguistic Studies: Acoustics and Perception (Poster Session)}

\author{
Kenneth de Jong, Chair \\ Department of Linguistics, Indiana University, 322 Memorial Hall, Bloomington, Indiana 47403
}

\section{Contributed Papers}

\begin{abstract}
All posters will be on display from 8:30 a.m. to 5:00 p.m.. To allow contributors an opportunity to see other posters, contributors of odd-numbered papers will be at their posters from 8:30 a.m. to 10:15 a.m. and contributors of even-numbered papers will be at their posters from 10:15 a.m. to 12:00 noon. To allow for extended viewing time, posters will remain on display until 5:00 p.m.
\end{abstract}

2aSC1. The acquisition of $L 2$ phonetic categories: Single-, two-, and multiple-category assimilation in Dutch learners of Spanish. Paola Escudero (Utrecht Univ./McGill Univ., Trans 10, 3512 JK Utrecht, The Netherlands, paola.escudero@let.uu.nl) and Paul Boersma (Univ. of Amsterdam, The Netherlands)

The perceptual acquisition of Spanish vowel categories by Dutch speakers is examined. One might think that these learners have few problems because Dutch has as many as 12 monophthongs in its vowel inventory, and Spanish has only 5. However, a perception experiment shows that Dutch learners make quite a few mistakes when identifying Spanish vowels. It was predicted that these errors exemplify three well-attested patterns in the initial state of $L 2$ acquisition: single-, two-, and multiplecategory assimilation. These patterns may be found in the categorization of Spanish /i//e/e/ /e/-/a/, and /u/-/o/, respectively. The strategies that the learners follow to solve the problems associated with these patterns are presented, as well as the development of phonetic categorization in the three scenarios. Ninety-six listeners were tested with a three-way experiment design. The stimuli were Spanish CVCs embedded in Dutch or Spanish carrier sentences. The first two experiments were designed to find out to what degree learners perceive $L 2$ sounds differently from $L 1$ sounds. The third experiment tested the development of the learners' $L 2$ categorization accuracy. The combined results of the three experiments show that the learners indeed manifest the three different assimilation patterns, each of which is solved by a different strategy.

2aSC2. Phonetic discrimination and non-native spoken-word recognition. Andrea Weber (CUNY Grad. Ctr., Speech and Hearing Sci., 365 Fifth Ave., New York, NY 10016, aweber@gc.cuny.edu) and Anne Cutler (Max Planck Inst. for Psycholinguist., Wundtlaan 1, 6525 XD Nijmegen, The Netherlands, anne.cutler@mpi.nl)

When phoneme categories of a non-native language do not correspond to those of the native language, non-native categories may be inaccurately perceived. This may impair non-native spoken-word recognition. Weber and Cutler investigated the effect of phonetic discrimination difficulties on competitor activation in non-native listening. They tested whether Dutch listeners use English phonetic contrasts to resolve potential competition. Eye movements of Dutch participants were monitored as they followed spoken English instructions to click on pictures of objects. A target picture (e.g., picture of a paddle) was always presented along with distractor pictures. The name of a distractor picture either shared initial segments with the name of the target picture (e.g., target paddle, /paedl/ and competitor pedal, /pEdl/) or not (e.g., strawberry and duck). Half of the targetcompetitor pairs contained English vowels that are often confused by Dutch listeners (e.g., /ae/ and /E/ as in "paddle-pedal"), half contained vowels that are unlikely to be confused (e.g., /ae/ and /aI/ as in "parrotpirate"). Dutch listeners fixated distractor pictures with confusable English vowels longer than distractor pictures with distinct vowels. The re- sults demonstrate that the sensitivity of non-native listeners to phonetic contrasts can result in spurious competitors that should not be activated for native listeners.

2aSC3. Perception of the English intrusive stops by Korean listeners. Jeong-Im Han (Dept. of English, Konkuk Univ., Hwayang-dong 1, Gwangjin-gu, Seoul 143-701, Korea, jhan@konkuk.ac.kr)

This paper reports results of an experiment examining Korean listeners' perception of English intrusive stops in nasal-obstruent clusters. The experiment tests (1) how often intrusive stops are perceived; (2) how language-specific syllable structure constraints influence the perception, given the fact that Korean does not allow consonant clusters in syllable onsets and codas; (3) whether even the perception of phonetic variables like intrusive stops, not phonemes, could be improved by learning. Ninety English non-words with a monosyllable structure of CVC1C2 were created, where $\mathrm{C} 1=/ \mathrm{m}, \mathrm{n}, \mathrm{N} /$, and $\mathrm{C} 2=/ \mathrm{p}, \mathrm{k}, \mathrm{s} /$. The stimuli including additional 90 filler items were recorded by three native English speakers and one representative data among them was given to three groups of native Korean listeners in terms of their English proficiency. Each was asked to monitor the target sounds [Warner and Weber, J. Phonetics 29, 23-52 (2001)]. The preliminary results show that identification of intrusive stops in English is totally dependent on Korean syllable structure, so even stimuli with strong acoustic cues were misparsed. Nonetheless, there's a high correlation between perception of intrusive stops and listeners' English proficiency, showing the possibility of the improvement of perception by learning.

2aSC4. Effects of consonantal context on perception of French rounded vowels by American English adults with and without French language experience. Erika S. Levy and Winifred Strange (Speech and Hearing Sci., City Univ. of New York, Grad. School and Univ. Ctr., 365 5th Ave., New York, NY 10016, erilev@earthlink.net)

This study extended Gottfried's [J. Phonetics 12, 91-114 (1984)] investigation of the effects of learning French on $\mathrm{AE}$ listeners categorial discrimination of the contrasts involving Parisian French vowels $/ y /, / \varnothing /$, /u/, and /i/. Vowels were presented in / rabVp/ and /radVt/ bisyllables embedded in carrier phrases by three different speakers in an AXB discrimination task. Two groups were tested: proficient $L 2$-French-speaking $\mathrm{AE}$ listeners (Exp) and non-French-speaking AE listeners (Inexp). Overall, the Exp group performed better than the Inexp group on $/ \mathrm{u}-\varnothing /, / \mathrm{y}-\mathrm{i} /$, and $/ \mathrm{y}-\varnothing /$ distinctions (mean errors: $\operatorname{Exp}=5 \%$, Inexp $=24 \%$ ). However, for $/ \mathrm{u}-\mathrm{y} /$, the groups did not differ $(\operatorname{Exp}=30 \%$ vs Inexp $=24 \%$ errors $)$. The findings may be explained by perceptual assimilation patterns, which are determined, in part, by consonantal context. The Inexp group confused /y/ with /i/ in bilabial context, but /y/ with /u/ in alveolar context, whereas the Exp group 
confused $/ y /$ with $/ \mathrm{u} /$, in both contexts. For all contrasts, the Inexp group performed better in bilabial than in alveolar context (16\% vs 32\% errors), whereas no context effect was revealed for the Exp group. The results suggest that learning a foreign language includes learning its coarticulatory rules.

2aSC5. Perception of English final stops by native Spanish and Russian listeners. Paul Argodale and Winifred Strange (CUNY Grad. Ctr., 365 Fifth Ave., New York, NY 10016)

Spanish and Russian speakers perception of word-final stops was investigated in an identification task. Natural stimulus triads (sue-suit-sued, see-seat seed) were produced and presented in two conditions: (1) Final consonants released and $\mathrm{CV}$ in the utterance final position; (2) final consonants unreleased and $\mathrm{CV}$ produced before a word beginning with a voiceless stop. Russian listeners, whose first language has final voiceless stops, correctly identified English final stops better than Spanish listeners, whose first language does not have final stops ( $72 \%$ vs $60 \%$ correct overall). Spanish listeners were more likely than Russian listeners to identify words with final stops as open syllables (31\% vs $2 \%$ errors). A significant effect of release condition was also found: Spanish listeners were much better at identifying released stops than unreleased stops (90\% vs $29 \%$ correct). Russian listeners also showed a slightly higher correct rate of identification for released stops ( $91 \%$ vs $42 \%)$. Both groups identified voiceless final stops more accurately than voiced stops: Russians $(80 \%$ vs $65 \%$ correct); Spanish (65\% vs $54 \%$ correct). These results replicate and extend earlier work by Flege and colleagues. [Work supported by a grant from the Professional Staff Congress of the City University of New York.]

2aSC6. Interlanguage intelligibility benefit as a function of talker and listener language background and $\boldsymbol{L} \mathbf{2}$ proficiency. Tessa Bent and Ann R. Bradlow (Dept. of Linguist., Northwestern Univ., 2016 Sheridan Rd., Evanston, IL 60208, t-bent@ northwestern.edu)

For non-native listeners, intelligibility of non-native speakers (NNS) can surpass intelligibility of native speakers [T. Bent, J. Acoust. Soc. Am. 109, 2472 (2001)]. The present study further investigated how language background and $L 2$ proficiency of talker and listener affect $L 2$ intelligibility. Two Chinese NNS, two Korean NNS, and one English monolingual were recorded reading simple English sentences. Listeners were English monolinguals, Chinese NNS, Korean NNS, and NNS with other $L 1 \mathrm{~s}$. For native English listeners, the native English talker was most intelligible. For non-native listeners, intelligibility of non-native talkers with high degrees of $L 2$ proficiency was better than or equal to native talker intelligibility. This pattern held regardless of whether non-native talkers and listeners matched in $L 1$. Additionally, the interlanguage benefit (i.e., the intelligibility difference between non-native and native talkers) decreased as the listeners' proficiency in English increased. This interlanguage benefit can be accounted for by a combination of a shared systematic interlanguage (when talker and listener match in $L 1$ ) and the influence of interlanguage universals (when talker and listener do not match in $L 1$ ). However, both of these factors become less influential as listeners $L 2$ proficiency develops causing a decrease in the interlanguage benefit. [Work supported by NIH-NIDCD Grant DC 03762.]

2aSC7. Phonological awareness of English by Chinese and Korean bilinguals. Hyunjoo Chung, Anna Schmidt, and Tse-Hsuan Cheng (School of Speech Pathol. and Audiol., Kent State Univ., Kent, OH 44242, aschmidt@kent.edu)

This study examined non-native speakers phonological awareness of spoken English. Chinese speaking adults, Korean speaking adults, and English speaking adults were tested. The L2 speakers had been in the US for less than 6 months. Chinese and Korean allow no consonant clusters and have limited numbers of consonants allowable in syllable final position, whereas English allows a variety of clusters and various consonants in syllable final position. Subjects participated in eight phonological awareness tasks (4 replacement tasks and 4 deletion tasks) based on English phonology. In addition, digit span was measured. Preliminary analysis indicates that Chinese and Korean speaker errors appear to reflect L1 influences (such as orthography, phonotactic constraints, and phonology). All three groups of speakers showed more difficulty with manipulation of rime than onset, especially with postvocalic nasals. Results will be discussed in terms of syllable structure, L1 influence, and association with short term memory.

2aSC8. Interaction of cues to vowel identity and consonant voicing: Cross-language perception. Geoffrey Stewart Morrison (Dept. of Linguist., Simon Fraser Univ., Burnaby, BC V5A 1S6, Canada)

Canadian English has two high front vowels differing in spectral and duration properties, Spanish has one high front vowel, and Japanese has two high front vowels differing in duration only. Vowel duration is a major cue to post-vocalic consonant voicing in English, but not in Japanese or Spanish. Canadian English, Japanese, and Mexican Spanish listeners identified members of a multidimensional edited speech continuum covering the English words bit, beat, bid, bead. The continuum was created by systematically varying the spectral properties of the vowel, and the durations of the vowel, the consonant closure, and the carrier sentence. English listeners had a categorical cutoff between /i/ and /I/ based primarily on the spectral properties of the vowel. Half the English listeners identified consonant voicing using vowel duration. Japanese listeners had a categorical cutoff between the English vowels based primarily on the duration of the vowel. The location of the cutoff was the same as the categorical cutoff between Japanese long /i:/ and short /i/. Japanese listeners identified consonant voicing at random. Spanish listeners identified the English vowels using vowel duration but did not have a categorical cutoff. Half the Spanish listeners identified consonant voicing using the spectral properties of the vowel.

2aSC9. Perception of English resyllabification by monolingual Japanese listeners. Kyoko Nagao, Byung-jin Lim, and Kenneth de Jong (Dept. of Linguist., Indiana Univ., Bloomington, IN 47405)

Previous results (Nagao et al., 2001) show that non-native listeners exhibit remarkably similar patterns of perceptual resyllabification with English listeners, suggesting that perceptual resyllabification is not a language specific phenomenon. The same listeners tended to identify voiced English tokens as voiceless, in keeping with Japanese voicing categories. In order to determine the degree to which the non-native perceptual resyllabification was due to extensive exposure to English, monolingual Japanese listeners, 8 from an older and 12 from a younger generation, participated in the same experiment. Monolinguals showed perceptual resyllabification of the same tokens as do English listeners', consistent with the previous results. Also consistent with previous results, especially older listeners' responses were more affected by Japanese voicing categories. In addition, older listeners were more likely than English listeners to identify tokens as CV's, while younger listeners were less likely to. The results for older listeners are what is expected of biases toward native categories. The results for the younger listeners, however, seem to indicate a developmental process involving the construction of new prosodic categories. [Work supported by NIDCD and NSF.]

2aSC10. Cross-language perception of fricative/vowel syllables by speakers of English and Mandarin. Sreedivya Radhakrishnan and Anna Schmidt (School of Speech Pathol. \& Audiol., P.O. Box 5190, Kent State Univ., Kent, OH 44242, sradhakr@kent.edu)

A recent study [Radhakrishnan and Schmidt (2001)] on the perception of fricative/vowel syllables by English and Mandarin speakers demonstrated that English speakers use the fricative noise while Mandarin speakers use both the fricative noise and vowel transitions in categorizing syl- 
lable initial fricatives in their native languages. In this study, crosslanguage mappings for fricative/vowel syllables were obtained from native English and Mandarin speakers. Native English and Mandarin speakers labeled and rated three sets of tokens from L2. These tokens were (1) fricative noises from four different vowel environments, (2) natural fricative vowel syllables, and (3) $\mathrm{F}+\mathrm{V}$ combinations in which the fricative noise from one language was combined with vowel transition from the other language. Vowel length was manipulated in the third set of stimuli. Results indicate differences in the cross-language mappings by the two language groups.

2aSC11. Learning to perceive English /d/ versus /ठ/: A comparison of French, English, and English-French bilingual 4-year-olds. Megha Sundara, Linda Polka (School of Commun. Sci. and Disord., McGill Univ., 1266 Pine Ave. W., Montreal, QC H3G 1A8, Canada), Lisa Campisi, Fred Genesee, and Caroline Marcoux (McGill Univ., Montreal, QC H3A 1B1, Canada)

Recent findings show that discrimination of the English /d-ð/ does not differ for English and French infants (6-8-month-olds and 10-12-montholds), although English adults clearly outperform French adults on this contrast, which is not phonemic in French. With respect to age effects, English listeners' perception of /d-ठ/ improves between infancy and adulthood, whereas French listeners' perception remains unchanged [Polka et al., J. Acoust. Soc. Am. 109, 2190-2200 (2001)]. In the present study, we tested monolingual English, monolingual French, and early EnglishFrench bilingual 4-year-olds on the same contrast using the same stimuli and procedures to clarify when facilitative effects of language experience emerge and whether they are affected by bilingualism. Four findings are reported. First, a language effect (English $>$ French) is evident by 4 years of age. Second, among native (English) listeners facilitative effects are evident by 4 -years of age (infants $<4$-year-olds $<$ adults). Third, among non-native (French) listeners discrimination performance is comparable across the age groups tested (infants $=4$-year-olds $=$ adults). Fourth, bilingual 4-year-olds' performance is virtually identical to that of their Frenchspeaking peers, revealing a strong effect of bilingualism on the perception of this contrast. Several factors contributing to these findings will be discussed.

2aSC12. On the role of phonetic inventory in the perception of foreign-accented speech. Joan Sereno, Joyce McCall, Allard Jongman (Linguist. Dept., The Univ. of Kansas, 1541 Lilac Ln., Lawrence, KS 66044-3177, sereno@ku.edu), Ton Dijkstra, and Walter van Heuven (Univ. of Nijmegen, 6500 HE Nijmegen, The Netherlands)

The current study investigates the effect of phonetic inventory on perception of foreign-accented speech. The perception of native English speech was compared to the perception of foreign-accented English (Dutch-accented English), with selection of stimuli determined on the basis of phonetic inventory. Half of the stimuli contained phonemes that are unique to English and do not occur in Dutch (e.g., [ $\theta]$ and [æ]), and the other half contained only phonemes that are similar in both English and Dutch (e.g., [s], [i]). Both word and nonword stimuli were included to investigate the role of lexical status. A native speaker of English and a native speaker of Dutch recorded all stimuli. Stimuli were then presented to 40 American listeners using a randomized blocked design in a lexical decision experiment. Results reveal an interaction between speaker (native English versus native Dutch) and phonetic inventory (unique versus common phonemes). Specifically, Dutch-accented stimuli with common phonemes were recognized faster and more accurately than Dutch-accented stimuli with unique phonemes. Results will be discussed in terms of the influence of foreign accent on word recognition processes.
2aSC13. Spectral resolution and English experience: effects on English phoneme and word recognition by non-native English speakers. Monica Padilla (Dept. of Biomed. Eng., Univ. of Southern California, Los Angeles, CA) and Robert V. Shannon (House Ear Inst., Los Angeles, CA)

Normal hearing listeners whose first language was Spanish were tested with English phonemes, words and sentences. Listeners were divided into four categories according to experience with the second language. Speech was presented in a sound treated booth at a level of $70 \mathrm{dBA}$. Listening conditions included noise (SNR of $15 \mathrm{~dB}, 10 \mathrm{~dB}, 5 \mathrm{~dB}, 0 \mathrm{~dB}$, and $5 \mathrm{~dB}$ ) and reduced spectral information (2, 4, 6, 8 and 16 frequency bands). Plomp's Model [J. Speech Hear. Res. 29, 146-154 (1986)] was applied to the data. The distortion factor " $D$ " defined by Plomp was found to increase with an increased loss of spectral resolution. It was also found to increase with age of learning of the second language. An additional "distortion" seems to be introduced when a second language is learned at a later age. Non-native listeners had more difficulty understanding vowels, words and sentences. Surprisingly, English experience had less effect on word and sentence recognition than on vowel recognition. Significantly lower performance on vowel recognition was seen even for fully bilingual listeners with reduced spectral resolution which could probably be related to the conflicting vowel spaces of the two languages. [Work funded by NIDCD.]

2aSC14. Language-specific effects in speaker and rate normalization. Allard Jongman, Yuwen Lai (Linguist. Dept., The Univ. of Kansas, 1541 Lilac Ln., Lawrence, KS 66044-3177, jongman@ku.edu), and Corinne Moore (ProQuest, Richfield, OH 44286)

This study concerns the effects of context on the perception of Mandarin tones. Mandarin tones are primarily distinguished in terms of $F 0$ range and temporal location of inflection point. The most relevant context effects therefore involve changes in speaker and speaking rate. In this study, we compared speaker and rate normalization of Mandarin tones 2 (mid-rising) and 3 (low-falling-rising) by Mandarin and English listeners. Synthetic tone 2 -tone 3 continua varying in turning point (TP, the inflection point of the tone), $\Delta F 0$ (the difference in $F 0$ between onset and turning point), or both were appended to natural precursor phrases representing high- and low-pitched speakers, as well as fast and slow speaking rates. Results of the rate experiments with Mandarin listeners confirmed that normalization occurs only when precursors and stimuli vary along the same acoustic dimension, a finding previously observed for speaker normalization [C. B. Moore and A. Jongman, J. Acoust. Soc. Am. 102, 18641877 (1997)]. English speakers showed speaker and rate normalization effects as well, but only for the continuum in which TP and $\Delta F 0$ varied simultaneously. We propose that limitations on perceptual resources allow English listeners to attend to extrinsic information only when intrinsic acoustic differences become more perceptually salient.

2aSC15. Effects of training on learning non-native speech contrasts. Joan M. Sinnott (Psych. Dept., Univ. of South Alabama, Mobile, AL 36688)

An animal psychoacoustic procedure was used to train human listeners to categorize two non-native phonemic distinctions. In Exp 1, Japanese perception of the English liquid contrast /r-1/ was examined. In Exp 2, American-English perception of the Hindi dental-retroflex contrast /d-D/ was examined. The training methods were identical in the two studies. The stimuli consisted of $64 \mathrm{CVs}$ produced by four different native talkers (two male, two female) using four different vowels. The procedure involved manually moving a lever to make either a "go-left" or "go-right" response to categorize the stimuli. Feedback was given for correct and incorrect responses after each trial. After 32 training sessions, lasting about 8 weeks, performance was analyzed using both percent correct and response time as measures. Results showed that the Japanese listeners, as a group, were statistically similar to a group of native listeners in categorizing the liquid contrast. In contrast, the Amercan-English listeners were not 
nativelike in categorizing the dental-retroflex contrast. Hypotheses for the different results in the two experiments are discussed, including possible subject-related variables. In addition, the use of an animal model is proposed to objectively "calibrate" the psychoacoustic salience of various phoneme contrasts used in human speech.

2aSC16. Listeners' perceptions of and attitudes toward Hispanic-, Asian-, and Arabic-accented English speakers. Norman Lass, Traci Atkins (Dept. of Speech Pathol. \& Audiol., West Virginia Univ., Morgantown, WV 26506-6122), and Rebekah Squires (James Madison Univ., Harrisonburg, VA 22807)

A master tape containing the randomized recordings of 20 Hispanic-, Asian-, and Arabic-accented English speakers reading a standard prose passage was presented to a group of 22 native English-speaking listeners who participated in two listening sessions. In the first session they were asked to use a 5-point listening preference rating scale. In the second session they heard the same speakers and were asked to determine the presence or absence of an accent and, if present, the type (Asian, Hispanic, Arabic, or other) and degree (mild, moderate, or severe) of accentedness. A scattergram plotting listeners' mean listening preference ratings and degree of accentedness ratings for each of the speakers in the study revealed a strong inverse relationship which yielded a statistically significant $(p<0.01)$ correlation coefficient.Thus, the higher the degree of severity of the listeners' judged accentedness of speakers, the more negative their listening preference rating judgments of the speakers. Implications of these findings and suggestions for future research are discussed.

2aSC17. An acoustical study of English word stress produced by Americans and Koreans. Byunggon Yang (English Dept., Dongeui Univ., 24 Kayadong, Pusanjingu, Pusan 614-714, South Korea)

Acoustical correlates of stress can be divided into duration, intensity, and fundamental frequency. This study examined the acoustical difference in the first two syllables of stressed English words produced by ten American and Korean speakers. The Korean subjects scored very high in TOEFL. They read, at a normal speed, a fable from which the acoustical parameters of eight words were analyzed. In order to make the data comparison meaningful, each parameter was collected at 100 dynamic time points proportional to the total duration of the two syllables. Then, the ratio of the parameter sum of the first rime to that of the second rime was calculated to determine the relative prominence of the syllables. Results showed that the durations of the first two syllables were almost comparable between the Americans and Koreans. However, statistically significant differences showed up in the diphthong pronunciations and in the words with the second syllable stressed. Also, remarkably high $r$-squared values were found between pairs of the three acoustical parameters, which suggests that either one or a combination of two or more parameters may account for the prominence of a syllable within a word. [Work supported by Korea Science Foundation R01-1999-00229.]

2aSC18. Perception of cross-language vowel differences: A longitudinal study of native Spanish learners of English. Satomi Imai (Div. of Speech and Hearing Sci., Univ. of Alabama at Birmingham, Birmingham, AL 35294, imais@uab.edu), James Flege (Univ. of Alabama at Birmingham, Birmingham, AL 35294), and Ratree Wayland (Univ. of Florida at Gainesville, Gainesville, FL 32611-5454)

The study evaluated the ability of native Spanish speakers to perceive phonetic differences between Spanish vowels (/i e a o u/) and English vowels $\left(/ \mathrm{ie}^{\mathrm{I}} \mathrm{ao}^{v} \mathrm{u} /\right.$ ). Eighteen adult native speakers of Spanish who were learning English as a second language (L2) in Birmingham. AL were tested at 6-month intervals over a 3.5-year period (T1-T7). Five tokens of each Spanish and English vowel were randomly presented for classification in terms of one of the five vowels of Spanish, and were rated for goodness of fit on a 6-point scale (where 0 indicated "not Spanish" and 5 indicated a "good example" of a Spanish vowel). At both T1 and T7, English $/ \mathrm{i} /, / \mathrm{e}^{\mathrm{I} /}, / \mathrm{a} /, / 0 \%$ and $/ \mathrm{u} /$ were usually classified as Spanish /i/, /e/, $/ \mathrm{a} /, / \mathrm{o} /$ and $/ \mathrm{u} /$, respectively. A group analysis revealed that significantly higher ratings were given to the Spanish than English member of the $/ \mathrm{o} /-/ \mathrm{o} \% /, / \mathrm{u} /-/ \mathrm{u} /, / \mathrm{e} /-/ \mathrm{e}^{\mathrm{I} /}$ and $/ \mathrm{a} /-/ \mathrm{a} /$ pairs but not the /i/-/i/ pair. However, the number of individual L2 learners who gave significantly higher ratings to a Spanish vowel than to the corresponding English vowel differed substantially $\left(/ \mathrm{o} /-/ \mathrm{o}^{\mathrm{o}} / n=18, \mathrm{u} /-/ \mathrm{u} / n=8\right.$, /e/-/e $/ \mathrm{I}^{\mathrm{I}} n=5, / \mathrm{a} /-/ \mathrm{a} / n=1$, /i/-/i/ $n=1)$. Possible explanations for these between-pair differences - acoustic and perceptual—will be discussed. [Work supported by NIH.]

2aSC19. Release bursts in English word-final stops: A longitudinal study of Korean adults' and children's production. Kimiko Tsukada (Div. of Speech \& Hearing Sci., Univ. of Alabama at Birmingham, CH20, 1530 3rd Ave. S., Birmingham, AL 35294, tsukadak@shrp.uab.edu), Molly Mack (Univ. of Illinois, Urbana, IL 61801), Hyekyung Sung (Stanford Univ., Stanford, CA 94305), David Birdsong (Univ. of Texas, Austin, TX 78712), and Ellen Bialystok (York Univ., Toronto, ON M3J 1P3, Canada)

Stops at the end of Korean words are always unreleased. The question addressed here was whether Korean adults and children living in the U.S. can learn to release stops at the end of English words. Four groups of 18 native Koreans (NK) who differed according to age (adult versus child) and length of residence in the U.S. (3 vs 5 years at T1) participated. Two native English (NE) groups served as age-matched controls. Production data were collected at two times (T1, T2) separated by one year. English words ending in $/ \mathrm{t} / \mathrm{and} / \mathrm{k} /$ were then examined in perception experiments (Exp. 1, Exp. 2). NE-speaking judges decided whether the final stop has a release burst or not. Exp. 1 showed that NE talkers released /t/ more often than NK talkers did. The effect of time was also significant. Talkers produced release bursts more often at T2 than at T1. Exp. 2 showed that, unlike Exp. 1, there were significant differences between NK adults and children. While NK children did not differ from NE children, NK adults released the final $/ \mathrm{k} /$ much less often than NE adults did. Possible reasons for why the expected children's advantage was seen for $/ \mathrm{k} /$, but not for $/ \mathrm{t} /$, will be discussed. [Work supported by NIH.]

2aSC20. An acoustic study of hiatus resolution in two Romance languages. Ioana Chitoran (Linguist. \& Cognit. Sci., Dartmouth College, HB 6087, Hanover, NH 03755) and Jose Ignacio Hualde (Univ. of Illinois at Urbana-Champaign, Urbana, IL 61801)

Spanish and Romanian contrast vowel sequences $[\mathrm{CiV}]$ in hiatus and corresponding diphthongs $[\mathrm{CjV}]$, with some interspeaker variation. Both languages contain surface diphthongs derived historically by gliding, $/ \mathrm{CiV} />[\mathrm{CjV}]$. They both show a strong tendency for blocking gliding word-initially, supported by native speaker judgments: Sp. [miope], Rom. [miopu] short-sighted; Sp. [italjana], Rom. [italjana] Italianf. Data from six speakers of each language confirmed this variation. The duration and $F 2$ transition rate of the vocalic sequence were compared, in words containing [i.a] and [ja]. [ia] was significantly longer in hiatus, and had a significantly slower transition rate than in [ja]. However, the ranges of the hiatus and [ja] sets showed some overlap, suggesting that hiatus resolution is not a categorical phonological process. Instead, lexical items fall on a hiatus-to-[ja] continuum. Further comparison of ranges and standard deviations confirmed the correlation between this variation and word position. In both languages more word-initial sequences resist gliding. Significantly less variation was found in the range for hiatus word-initially than for [ja] word-medially. This suggests that the combination of lingual gestures between high and nonhigh vowels is more tightly controlled wordinitially than word-medially, a result previously reported for stop sequences [Byrd (1996); Chitoran, Goldstein, and Byrd (unpublished)]. 
2aSC21. Segmental and coarticulatory timing in Ikalanga prenasalized stops. Patrice S. Beddor, Chutamanee Onsuwan, and Rose Letsholo (Dept. of Linguist., Univ. of Michigan, Ann Arbor, MI 48109, beddor@umich.edu)

The temporal characteristics of prenasalized stops in Ikalanga, a Bantu language of Botswana, are investigated. Eight speakers were recorded producing Ikalanga words with $\mathrm{V}^{\mathrm{N}} \mathrm{CV}\left({ }^{\mathrm{N}} \mathrm{C}=/ \mathrm{m}^{\mathrm{m}} \mathrm{b}^{\mathrm{n}} \mathrm{d} \mathrm{\eta}_{\mathrm{g}}\right)$, VNV $(\mathrm{N}=/ \mathrm{mnn})$, and $\mathrm{VCV}(\mathrm{C}=/ \mathrm{b} \mathrm{d} \mathrm{g} /)$ sequences. Acoustic measures of 3 speakers' productions showed that oral closure durations (from vowel offset to closure release) were only slightly longer for ${ }^{\mathrm{N}} \mathrm{C}$ than for $\mathrm{N}$ or $\mathrm{C}(94,83$, and 83 $\mathrm{ms}$, respectively). When analyzed separately, the ${ }^{\mathrm{N}}$ and $\mathrm{C}$ portions of $\mathrm{V}^{\mathrm{N}} \mathrm{CV}$ were somewhat shorter than their counterparts in VNV and VCV, but nearly all of the shortening occurred in the $\mathrm{C}$ portion (in $\mathrm{V}^{\mathrm{N}} \mathrm{CV}$, $\mathrm{N}=78 \mathrm{~ms}$ and $\mathrm{C}$ closure $=16 \mathrm{~ms}$ ). The durations of vowels preceding the medial consonants did not differ across the three consonant types. Thus, for segmental durations, the main difference between Ikalanga $\mathrm{V}^{\mathrm{N}} \mathrm{CV}$ and VNV was the presence of a brief oral occlusion in the former. However, measures of coarticulatory nasalization point to another systematic difference: while both sequences exhibited comparable anticipatory vowel nasalization, VNV (but not $\mathrm{V}^{\mathrm{N}} \mathrm{CV}$ ) sequences showed carryover nasalization extending through more than $70 \%$ of the post-nasal vowel. Findings will be compared with previous work on Bantu prenasalized stops. [Work supported by NSF.]

2aSC22. Factors affecting the duration of native and second-language sentences produced in unspeeded and speeded conditions. James E. Flege (Div. of Speech and Hearing Sci., Univ. of Alabama at Birmingham, Birmingham, AL 35294, jeflege@uab.edu) and Ian R. A. MacKay (Univ. of Ottawa, Ottawa, ON K1N 6N5, Canada)

The aim of this study was to provide insight into why non-natives generally produce longer second-language (L2) sentences than native speakers do. Four groups of 16 Italian-English bilinguals were recruited in Ottawa based on orthogonal differences in age of arrival (AOA) to Canada from Italy (early versus late) and self-reported percentage Italian use (low-L1 use versus high-L1 use). The bilinguals repeated durationmatched English and Italian sentences presented via a loudspeaker in an "unspeeded" condition, then as rapidly as possible (the "speeded" condition). The same effect of AOA was obtained in both conditions despite a $20 \%$ reduction in sentence duration in the speeded condition. That is, the early bilinguals produced significantly shorter English than Italian sentences, whereas the late bilinguals produced significantly longer English than Italian sentences. Different L1 use effects were obtained in the two conditions, however. In the unspeeded condition, the low-L1-use bilinguals produced shorter English than Italian sentences, with a nonsignificant effect of language for high-L1-use bilinguals. In the speeded condition, high-L1-use bilinguals produced shorter Italian than English sentences, with no language effect for the low-L1-use bilinguals. The underlying bases of AOA and L1 use effects on L2 sentence production will be discussed. [Work supported by NIH.]

2aSC23. Duration and $F \mathbf{0}$ cues for vowel length in Japanese. Tomoko Kozasa (Dept. of Linguist., Univ. of Hawaii at Manoa, 1890 East-West Rd., Moore Hall 596, Honolulu, HI 96822)

Two acoustic cues for the long-short vowel contrast were investigated in productions of Japanese accented vowels under two rates of speech. An accented word has a high-low pitch sequence. Long accented vowels have the fall within the syllable, while short vowels do not. Unsurprisingly, the vowel duration was significantly shorter in fast speech than in slow speech overall. The speech rate affected the long vowel duration more than the short vowel duration; the ratio between short vowels and long vowels varied by speech rate: 1:1.7 in fast speech and 1:1.9 in slow speech. However, the pitch fall was not significantly affected by the speech rate in either type of vowel. These results suggest that for native Japanese speakers, pitch information may be a more stable cue than duration for distinguishing the length of accented vowels. A perception study manipulating these cues is in progress. Synthesized long vowels without the pitch fall characteristic of long accented vowels are compared with normally accented long and short vowels. If Japanese speakers perceive the synthesized vowels as short, this constitutes evidence that for accented vowels, the pitch cue dominates the duration cue.

2aSC24. Acquisition of Japanese contracted sounds in L1 phonology. Chiharu Tsurutani (School of Lang. and Linguist., Griffith Univ., Nathan 4111 QLD, Australia, C.Tsurutani@mailbox.gu.edu.au)

Japanese possesses a group of palatalized consonants, known to Japanese scholars as the contracted sounds, $[\mathrm{CjV}]$. English learners of Japanese appear to treat them initially as consonant + glide clusters, where there is an equivalent $[\mathrm{Cj}]$ cluster in English, or otherwise tend to insert an epenthetic vowel $[\mathrm{CVjV}]$. The acquisition of the Japanese contracted sounds by first language (L1) learners has not been widely studied compared with the consonant clusters in English with which they bear a close phonetic resemblance but have quite a different phonological status. This is a study to investigate the L1 acquisition process of the Japanese contracted sounds (a) in order to observe how the palatalization gesture is acquired in Japanese and (b) to investigate differences in the sound acquisition processes of first and second language (L2) learners: Japanese children compared with English learners. To do this, the productions of Japanese children ranging in age from 2.5 to 3.5 years were transcribed and the pattern of misproduction was observed.

2aSC25. Acoustic and perceptual evidence of complete neutralization of word-final tonal specification in Japanese. Kazumi Maniwa (Dept. of Linguist., Univ. of Kansas, 1541 Lilac Ln., 427 Blake, Lawrence, KS 66044-3177)

This study investigated the extent to which the Japanese lexical pitchaccent distinction is neutralized in word-final position. Native speakers of Tokyo Japanese produced minimal word pairs differing in final accent status. Words were produced both in isolation and in a sentential context, where neutralization would not be expected due to following tonal specification. Examination of pitch patterns on relevant moras revealed a clear distinction between accent-opposed pairs produced in context but no such difference between items produced in isolation. Both the words produced in isolation and the words excised from sentential contexts were then presented to Japanese listeners in a lexical identification task. Participants could clearly distinguish items extracted from sentences but identified words uttered in isolation at chance level. These results suggest that phonological neutralization of final pitch accent is complete, showing no effects of underlying specification in either production or perception.

2aSC26. Perception and production of word-initial long fricatives in Polish. Ela Thurgood (Dept. of English, California State Univ., Chico, CA 95929-0830)

The paper provides an analysis of the perception and production of the Polish distinction between long fricative /v:/ and short fricative /v/ in pre-vocalic position. The task involved 27 native speakers of Polish repeating 50 sentences, including the target sentences. The target sentences were minimal pairs with the meaning difference based solely on fricative length. The results showed that the 190-ms mean duration of the long fricatives was well over twice as long as the 71-ms duration of the short fricatives. When duration of the fricative fell below $147 \mathrm{~ms}$, the listener's ability to discriminate between a short and long fricative was significantly reduced. Fricative length correlated with the duration of the following vowel: $78 \%$ of the time, the vowel was longer in the syllable with the long fricative. Indeterminacy about the identification of long fricatives is particularly well marked in these data: $42 \%$ of the responses to prompts with long fricatives included a distinctive pitch pattern, a so-called flat hat: a rising $\mathrm{F} 0$ in the first syllable, pitch height maintenance, and a drop after the second syllable. In Polish, this pattern indicates that the speaker is paying particular attention to this part of the utterance [Demenko (1999)]. 
2aSC27. Tone classification of syllable-segmented Thai speech based on multilayer perception. Nuttavudh Satravaha, Powsiri Klinkhachorn (Ln. Dept of Computer Sci. \& Elec. Eng., West Virginia Univ., Morgantown, WV 26506), and Norman Lass (West Virginia Univ., Morgantown, WV 26506)

Thai is a monosyllabic tonal language that uses tone to convey lexical information about the meaning of a syllable. Thus to completely recognize a spoken Thai syllable, a speech recognition system not only has to recognize a base syllable but also must correctly identify a tone. Hence, tone classification of Thai speech is an essential part of a Thai speech recognition system. Thai has five distinctive tones ("mid," "low," "falling," "high," and "rising") and each tone is represented by a single fundamental frequency $(F 0)$ pattern. However, several factors, including tonal coarticulation, stress, intonation, and speaker variability, affect the $F 0$ pattern of a syllable in continuous Thai speech. In this study, an efficient method for tone classification of syllable-segmented Thai speech, which incorporates the effects of tonal coarticulation, stress, and intonation, as well as a method to perform automatic syllable segmentation, were developed. Acoustic parameters were used as the main discriminating parameters. The $F 0$ contour of a segmented syllable was normalized by using a $z$-score transformation before being presented to a tone classifier. The proposed system was evaluated on 920 test utterances spoken by 8 speakers. A recognition rate of $91.36 \%$ was achieved by the proposed system.

2aSC28. An acoustical study of the voicing distinction in Dutch plosives. Petra M. van Alphen (MPI for Psycholinguist., P.O. Box 310, 6500 AH Nijmegen, The Netherlands, petra.vanalphen@mpi.nl)

Dutch has two voiced plosives, namely $/ \mathrm{b} /$ and $/ \mathrm{d} /$. They are said to have a negative VOT (i.e., are prevoiced), while the voiceless plosives $/ \mathrm{p} /$ and $/ \mathrm{t} /$ have a small, positive VOT. A production study was designed to investigate two questions. First, how does prevoicing vary in spoken Dutch? Second, what other cues in the acoustic signal might contribute to the perceptual distinction between voiced and voiceless plosives? Ten subjects were asked to produce a list of monosyllabic words which were chosen to study the following factors: place of articulation (labial versus alveolar), following phoneme (vowel versus consonant), lexical status of the carrier stimulus (word versus nonword), and lexical competitor environment of the carrier stimulus. Results will be reported on the relative contribution of various durational, spectral, and energy measures to the voiced-voiceless distinction. The data showed that $25 \%$ of all tokens were produced without prevoicing. The prevoicing of the voiced plosive was omitted more often when the plosive was followed by a consonant than when it was followed by a vowel. Although both spectral and energy cues signal the voicing distinction, and although prevoicing is often omitted, VOT appears to be the primary cue to this distinction.

2aSC29. Using the Speech Transmission Index to predict the intelligibility of non-native speech. Sander J. van Wijngaarden, Herman J. M. Steeneken, Tammo Houtgast, and Adelbert W. Bronkhorst (TNO Human Factors, P.O. Box 23, 3769 ZG Soesterberg, The Netherlands)

The calibration of the Speech Transmission Index (STI) is based on native speech, presented to native listeners. This means that the STI predicts speech intelligibility under the implicit assumption of fully native communication. In order to assess effects of both non-native production and non-native perception of speech, the intelligibility of short sentences was measured in various non-native scenarios, as a function of speech-tonoise ratio. Since each speech-to-noise ratio is associated with a unique STI value, this establishes the relation between sentence intelligibility and STI. The difference between native and non-native intelligibility as a function of STI was used to calculate a correction function for the STI for each separate non-native scenario. This correction function was applied to the STI ranges corresponding to certain intelligibility categories (badexcellent). Depending on the proficiency of non-native talkers and listeners, the category boundaries were found to differ from the standard (native) boundaries by STI values up to 0.30 (on the standard $0-1$ scale). The corrections needed for non-native listeners are greater than for non-native talkers with a similar level of proficiency. For some categories of nonnative communicators, the qualification excellent requires an STI higher than 1.00 , and therefore cannot be reached.

2aSC30. Acoustic correlates of Georgian ejectives. Tamra M. Wysocki (Dept. of Linguist., Univ. of Chicago, 1050 E. 59th St., Chicago, IL 60637, t-wysocki@uchicago.edu)

In this paper we present results from acoustic analysis of Georgian ejectives. Georgian, a language of the Kartvelian (South Caucasian) family, has a three-way opposition for voiced, voiceless aspirated and ejective stops. There have not been many acoustic studies of ejectives; those studies that discuss ejectives, such as by Lindau [J. Phonetics 12, 147-155 (1984)], report cross-linguistic variation in timing between oral and glottal releases and the onset of a following vowel. In this paper we investigate acoustic correlates of Georgian ejectives and examine how these correlates are realized in two-member ejective clusters. Additionally, correlates of Georgian ejectives are compared to findings from previous studies and discussed in relation to cross-linguistic tendencies and variation. Data consist of field recordings of three native Georgian speakers producing single ejectives and ejective clusters in word-initial and word-medial (intervocalic) positions at a normal rate of speech. Acoustic analysis was completed using spectrograms and waveforms. Results indicate that, while there is variation within and among speakers, characteristics correlated with singleton Georgian ejectives include relative burst amplitude, noise quality following oral release, long, positive voice onset time, and a short period of creaky voice at the onset of a following vowel. Some of these characteristics differ from those of ejective clusters.

2aSC31. Japanese adult's and children's production and perception of English fricatives: A longitudinal study. Katsura Aoyama (Div. of Speech and Hearing Sci., Univ. of Alabama, Birmingham, CH20, 1530 3rd Ave. S., Birmingham, AL 35294, aoyama@uab.edu), Susan Guion (Univ. of Oregon, Eugene, OR 97403), Tsuneo Yamada (National Inst. of Multimedia Education, Chiba 261-0014, Japan), and Reiko AkahaneYamada (ATR Human Information Processing Res. Labs., Kyoto 619-0288, Japan)

This study examined the production and perception of English fricatives by native Japanese (NJ) adults and children (16 per group, mean age $=40$ and 10 years), and age-matched native English (NE) adults and children (16 per group). The subjects were tested two times (T1, T2) 1 year apart. (At T1, the NJ subjects' mean length of residence in the U.S. was 0.5 year.) A picture-naming task was used to elicit the production of English words beginning with $/ \mathrm{s} /$ and $/ \theta /$, and intelligibility scores were obtained for both. The intelligibility scores of the NJ children but not adults improved significantly from $\mathrm{T} 1$ to $\mathrm{T} 2$. The $\mathrm{NJ}$ children obtained significantly lower scores than the $\mathrm{NJ}$ adults did at $\mathrm{T} 1$, but at $\mathrm{T} 2$ the adult-child difference was nonsignificant. The perception of $/ \mathrm{s} /$ and $/ \theta /$ was tested by a categorial discrimination task. Although the NJ adult's and children's scores improved from $\mathrm{T} 1$ to $\mathrm{T} 2$, the $\mathrm{T} 1-\mathrm{T} 2$ differences were nonsignificant. Thus, the results showed that the NJ children's production scores improved significantly from $\mathrm{T} 1$ to $\mathrm{T} 2$, while there was no significant change for the perception scores on the discrimination of $/ \mathrm{s} /-/ \theta /$. The relationship between production and perception in L2 speech learning will be discussed. [Work supported by NIH.]

2aSC32. Temporal features of Spanish-accented English. Amee Shah and Winifred Strange (Ph.D. Prog. in Speech and Hearing Sci., City Univ. of New York, 365 Fifth Ave., New York, NY 10016)

In this study we attempt to identify the acoustic parameters of Spanish accented English that give it the perception of being accented. Recordings of eight multisyllabic (3, 4, and 5 syllables) words spoken in sentences by 22 Spanish speakers of English and five native speakers of American English (AE) were analyzed for temporal acoustic differences. Segmental durations of Spanish productions, including differences of stressedunstressed (s/u) vowel duration ratios, Voice Onset Time (VOT) and flap/ 
stop duration, differed from native productions. The sentences and the excised target words were given to native (AE) listeners $(N=10)$ who judged the degree of accentedness on a 9-point scale. The degree of accentedness on target words correlated with perceived ratings on the eight sentences (i.e., global accentedness), rho $=+0.82$. Spearman rank-order correlations between s/u vowel duration ratios and native listener ratings of accentedness on target words varied from +0.04 to 0.68 . The VOT duration of initial voiceless stops correlated positively with accentedness ratings for three of the four words $(+0.26$ to +0.35$)$. Overall, results suggest that Spanish-accented English is characterized by significant temporal differences from native American English, and that these temporal differences contribute to the perception of accentedness as judged by native American English listeners.

2aSC33. Perception and analysis of Spanish accents in English speech. Cori Chism (Dept. of Foreign Lang., West Virginia Univ., Morgantown, WV 26506) and Norman Lass (West Virginia Univ., Morgantown, WV 26506)

The purpose of the present study was to determine what relates most closely to the degree of perceived foreign accent in the English speech of native Spanish speakers: intonation, vowel length, stress, voice onset time (VOT), or segmental accuracy. Nineteen native English speaking listeners rated speech samples from 7 native English speakers and 15 native Spanish speakers for comprehensibility and degree of foreign accent. The speech samples were analyzed spectrographically and perceptually to ob- tain numerical values for each variable. Correlation coefficients were computed to determine the relationship beween these values and the average foreign accent scores. Results showed that the average foreign accent scores were statistically significantly correlated with three variables: the length of stressed vowels $(r=-0.48, p=0.05)$, voice onset time $(r$ $=-0.62, p=0.01)$, and segmental accuracy $(r=0.92, p=0.001)$. Implications of these findings and suggestions for future research are discussed.

2aSC34. A cross-language study of vowel sounds produced with and without emphasis: Testing the theory of adaptive dispersion. Jessica F. Hay, Momoko Sato, Amy E. Coren, and Randy L. Diehl (Dept. of Psych., Univ. of Texas, Austin, TX 78712)

According to Lindbloms Theory of Adaptive Dispersion (TAD), the aim of talkers is to make phonological contrasts sufficiently distinctive to promote linguistic comprehension by the listener while minimizing the articulatory effort needed to achieve this degree of distinctiveness. When part of an utterance carries new-rather than given-information, it tends to be spoken with greater emphasis and clarity. In this study, several possible acoustic correlates of vowels in emphasized words were examined in American English, French, and Japanese in comparable phonetic and sentence contexts. These possible correlates include an expanded vowel space, greater vowel inherent spectral change, and a greater systematic variation in vowel length. Preliminary analyses suggest that the contrastenhancing properties of emphasized vowels vary considerably across languages. [Work supported by NIDCD.]

TUESDAY MORNING, 4 JUNE 2002

BENEDUM ROOM, 9:25 TO 10:40 A.M.

\title{
Session 2aSPa
}

\section{Signal Processing in Acoustics: Applications of Time Reversal Processing}

\author{
Leon H. Sibul, Chair \\ Applied Research Laboratory, Pennsylvania State University, P.O. Box 30, State College, Pennsylvania 16804
}

Contributed Papers

9:25

2aSPa1. Effectiveness of using time reverse signal processing for mitigating multipath in communications channels. Alan W. Meyer, James V. Candy, and Andrew J. Poggio (Lawrence Livermore Natl. Lab., 7000 East Ave., Livermore, CA 94550)

The use of time reverse signal processing (TRSP) to compensate for distortion in a communications channel is well established in recent literature. Our recent work quantifies the effectiveness of TRSP techniques vis-à-vis traditional adaptive equalization techniques. These quantitative measurements are developed using simulations and laboratory experiments. A rigorous mathematical development of the communications problem is first necessary to establish a consistent model of the communications channel, and various approaches for implementing TRSP and adaptive equalization as well. These techniques are then simulated and performance measures developed for a large set of conditions spanning an increasing number of multipaths and increasing SNR. Acoustic laboratory experiments are also conducted in a highly reverberant environment to confirm both our techniques and results. In this paper we report on these measures of effectiveness comparing TRSP vis-a-vis traditional adaptive equalization techniques for compensating for multipath in a communications channel. [Work performed under the auspices of the Department of Energy by the Lawrence Livermore National Laboratory under contract W-7405-Eng-48.]
9:40

2aSPa2. Broadband DORT applied to bistatic active sonar. David M. Fromm and Charles F. Gaumond (Naval Res. Lab., Washington, DC 20375-5350)

The decomposition of the time reversal operator (DORT) is a single frequency method of spatially isolating scatterers with a multiple-source/ multiple-receiver system [C. Prada et al., J. Acoust. Soc. Am. 99, 20672076 (1996)]. The DORT technique applied to the active sonar problem has demonstrated the ability to isolate scatterers at various depths and ranges from distributed bottom reverberation for a set of monostatic or vertically bistatic source/receiver geometries. The sound channel propagation has several consequences for DORT analysis: (1) frequency fading prevents the source and receiving steering vectors for the first singular value from isolating the scatterer across the entire bandwidth of the signal; and (2) dispersion causes space-time spreading of the analysis window and complicates the back-propagation procedure. These effects become more pronounced as the technique is extended to fully bistatic sourcereceiver geometries and range-dependent environments. This paper presents the extension of the DORT technique to the full broadband, bistatic, active sonar problem, including refinements to the range-resolution/timewindowing and back-propagation techniques, and enhancement to detection and classification by exploiting the response for several singular values across the entire bandwidth of the signal. [Work supported by ONR.] 
an eigenstate of the time reversal operator associated with either a void or the interface. The eigenstates associated with the interface can be understood in terms of interactions between the array and its image in the interface. We look at the problem of detecting eigenstates associated with voids in the presence of the interface eigenstates. Strategies for classifying the eigenstates are derived by considering the simplified problem of a time reversal array on a single layer slab. [Work performed under the auspices of the Department of Energy by the Lawrence Livermore National Laboratory under contract No. W-7405-Eng-48.]

\section{$10: 25$}

2aSPa5. Buried target detection using time reversal in inhomogeneous sediment. David Pierson and Thomas Drake (Marine, Earth and Atmospheric Sci., North Carolina State Univ., Raleigh, NC 27695)

Time reversal has been demonstrated to refocus acoustic waves in homogeneous media with multiple scatters. It has also been shown to work through a liquid-solid interface when both media are homogeneous. We will show that time reversal can also work to detect objects in inhomogeneous solids. By modeling a single transducer in water located $10 \mathrm{~m}$ above the water-solid interface we will show the location of the object can be isolated using an iterative time reversal method. Simulations are performed with the solid medium having a mean density of quartz sand varying spatially with horizontal correlation length $0.5 \mathrm{~m}$ and vertical correlation length $0.02 \mathrm{~m}$. A sphere is buried in the sand having a density of iron in one test and a density closer to quartz sand in another. The sphere diameter was changed in each simulation ranging from $9 \mathrm{~cm}$ to $19 \mathrm{~cm}$. The initial pulses were sinusoidal with frequencies of $10 \mathrm{kHz}, 100 \mathrm{kHz}$, and $500 \mathrm{kHz}$. Results show improved localization of the sphere. It will also be shown that the iterative time reversal method goes to a response that is independent of initial pulse frequency as predicted by previous theories. [Research supported by ONR.]

TUESDAY MORNING, 4 JUNE 2002

BENEDUM ROOM, 10:50 TO 11:55 A.M.

\title{
Session 2aSPb
}

\section{Signal Processing in Acoustics and Archives and History: History of Signal Processing in Acoustics}

\author{
James V. Candy, Chair \\ Lawrence Livermore National Laboratory, P.O. Box 808, Livermore, California 94551 \\ Chair's Introduction-10:50 \\ Invited Paper
}

\begin{abstract}
2aSPb1. History of signal processing in acoustics. David I. Havelock (Natl. Res. Council, 1200 Montreal Rd., Ottawa, ON K1A 0R6, Canada, david.havelock@nrc.ca)

The Technical Committee on Signal Processing in Acoustics is the youngest of the ASA's 13 Technical Committees. It was formed as an Interdisciplinary Technical Group in Fall 1994 and was converted to a Technical Committee in Fall 2000. It has had over 120 technical sessions, cosponsoring sessions with all of the other Technical Committees. Signal processing first appeared as a distinct PACS classification in 1964, but significant papers on signal processing appear in JASA well before this. The popularization of the fast Fourier transform and developments in digital computer technology spurred research and education in signal processing. In more recent years, advances in digital technology have allowed digital signal processing to advance beyond "off-line" simulation and analysis to embedded components of everyday devices and systems. As signal-processing capabilities advance, increased opportunities for cross-disciplinary applications can be expected. Accordingly, an important role for the Technical Committee on Signal Processing in Acoustics will be to enhance this interchange within the ASA. The history of signal processing in acoustics will be presented through examples from various areas of acoustics.
\end{abstract}




\title{
Session 2pAA
}

\section{Architectural Acoustics and Noise: Computer Modeling in Acoustical Consulting I}

\author{
Lily M. Wang, Chair \\ Architectural Engineering, University of Nebraska, 200B Peter Kiewit Institute, 1110 South 67th Street, \\ Omaha, Nebraska 68182-0681
}

Chair's Introduction-1:00

Invited Papers

1:05

2pAA1. Computer modeling in the practice of acoustical consulting: An evolving variety of uses from marketing and diagnosis through design to eventually research. Gary S. Madaras (RPG Diffusor Systems, Inc., 651-C Commerce Dr., Upper Marlboro, MD 20774)

The use of computer modeling as a marketing, diagnosis, design, and research tool in the practice of acoustical consulting is discussed. From the time it is obtained, the software can be used as an effective marketing tool. It is not until the software basics are learned and some amount of testing and verification occurs that the software can be used as a tool for diagnosing the acoustics of existing rooms. A greater understanding of the output types and formats as well as experience in interpreting the results is required before the software can be used as an efficient design tool. Lastly, it is only after repetitive use as a design tool that the software can be used as a cost-effective means of conducting research in practice. The discussion is supplemented with specific examples of actual projects provided by various consultants within multiple firms. Focus is placed on the use of CATT-Acoustic software and predicting the room acoustics of large performing arts halls as well as other public assembly spaces.

$1: 35$

2pAA2. Recent emphasis of computer modeling in architectural acoustics consulting. Ted Pyper, Derrick Knight, and Andrew Schmidt (Jaffe Holden Acoust., 114A Washington St., Norwalk, CT 06854, tpyper@jhacoustics.com)

Jaffe Holden Acoustics has been designing successful performing arts spaces for over four decades. Within recent years, the use of computer modeling programs, such as CATT Acoustic, has gained wide acceptance as a design tool in acoustical consulting. JHA has found computer modeling to be useful in refining some specific design issues. Models will be presented for Zankel Recital Hall at Carnegie Hall, Kennedy Center Concert Hall, Marion Oliver McCaw Hall in Seattle, and the University of Connecticut band rehearsal room. The purpose and application of the models will be discussed, along with the conclusions drawn from each study.

2pAA3. Computer modeling/auralization: Can these computer aids assist architects and owners in making proper project decisions? Robert C. Coffeen (School of Architecture and Urban Design, Marvin Hall, The Univ. of Kansas, Lawrence, KS, coffeen@ku.edu)

Computer modeling of architectural spaces and subsequent auralization provides the acoustical consultant with information which is not easily obtained by other techniques and methods, and which leads to better acoustical designs. But, what information does modeling/auralization produce which will help the architect and facility owner make proper decisions relating to room acoustics and electro-acoustics when such decisions have economic and architectural implications? This paper will present several examples of how modeling/auralization will assist in this quest for acoustical understanding and suitable project decisions.

2:35

2pAA4. Applications of computer modeling at Wrightson, Johnson, Haddon \& Williams, Inc. James A. Johnson and Benjamin C. Seep (WJHW, Inc., 4801 Spring Valley Rd., \#300, Dallas, TX 75244, jjohnson@wjhw.com)

Computer modeling has become useful as an investigative tool and as a client communication and explanation tool in the field of acoustical consulting. A variety of in-house developed and commercially available applications is in constant use at the firm of Wrightson, Johnson, Haddon \& Williams. Examples likely to be demonstrated (depending on time) include use of digital filtering for building exterior noise reduction comparisons, a shell isolation rating (SIR) model, simple sound barrier programs, an HVAC spreadsheet, a visual sightline modeling tool, specular sound reflections in a semicircular arc, and some uses of CATT-acoustic auralizations. 
2pAA5. Utilizing computer models for optimizing classroom acoustics. Jennifer M. Hinckley and Carl J. Rosenberg (Acentech, Inc., 33 Moulton St., Cambridge, MA 02138, jhinckley@acentech.com)

The acoustical conditions in a classroom play an integral role in establishing an ideal learning environment. Speech intelligibility is dependent on many factors, including speech loudness, room finishes, and background noise levels. The goal of this investigation was to use computer modeling techniques to study the effect of acoustical conditions on speech intelligibility in a classroom. This study focused on a simulated classroom which was generated using the CATT-acoustic computer modeling program. The computer was utilized as an analytical tool in an effort to optimize speech intelligibility in a typical classroom environment. The factors that were focused on were reverberation time, location of absorptive materials, and background noise levels. Speech intelligibility was measured with the Rapid Speech Transmission Index (RASTI) method.

3:50

2pAA6. A case-study comparison of computer modeling and scale modeling in acoustics consulting. Paul T. Calamia (Kirkegaard Assoc., 801 W. Adams St., 8th Fl., Chicago, IL 60607)

As an alternate or compliment to computer models, acoustics consultants often make use of scale models to evaluate the efficacy of architectural designs. The intention of this paper is to compare the two modeling approaches, using one or more case studies, to explore the pros and cons of each. Topics of comparison will include cost, geometric representations, effective bandwidths, propagation phenomena (e.g., diffraction), simulation of material properties, and auralization. Where possible, measured data from existing spaces will be presented to provide a reference for the modeled data.

4:20

2pAA7. Practical considerations in using audible simulation technology. Thomas Tyson and Kenneth Jacob (Bose Professional Systems Div., The Mountain, Framingham, MA 01701)

Ideally, consultants and clients alike would like to hear sound from computer models that is indistinguishable from the sound of the finished project. No one, however, would claim perfection in computer modeling, and differences between the model and the finished project certainly remain. How can practitioners in the field know exactly what these differences are, and more importantly, how they may affect the outcome of their work? Said a different way, how much can they and their clients trust what they hear from their computer models? In the work presented here, an approach used to answer these questions in the case of Boses Modeler and Auditioner programs is given. The approach is explained using: (1) laboratory results that focus on the critical dimension of speech intelligibility and (2) comparisons of actual system installations from the past seven years that were modeled and presented to end users using the Auditioner system.

TUESDAY AFTERNOON, 4 JUNE 2002

LE BATEAU ROOM, 1:00 TO 2:35 P.M.

\title{
Session 2pAB
}

\section{Animal Bioacoustics: Session on Marine Mammal Bioacoustics in Honor of William E. Evans II}

\author{
Ann E. Bowles, Cochair \\ Hubbs-Sea World Research Institute, 2595 Ingraham Street, San Diego, California 92109 \\ Jeanette A. Thomas, Cochair \\ Laboratory of Sensory Biology, Western Illinois University, 3561 60th Street, Moline, Illinois 61265
}

Chair's Introduction-1:00

\section{Contributed Papers}

1:05

2pAB1. A window on perception: Response times of odontocete cetaceans in audiometric tests. Diane J. Blackwood (Marine Acoust. Lab., Texas A\&M Univ. and 3027 Macaulay St., San Diego, CA 92106, blackwood@cls.org), Sam H. Ridgway (Navy Marine Mammal Prog., San Diego, CA 92152), and William E. Evans (Marine Acoust. Lab., Texas A\&M Univ.)

A standard psychometric measurement is response time, the interval elapsing between a stimulus and a response. While studies of response time have been published for humans and other terrestrial mammals, this study marks the first report of response times for odontocete cetaceans at threshold in an audiometric task. Two white whales (Delphinapterus leucas) and four Atlantic bottlenose dolphins (Tursiops truncatus) were given audiometric tests to determine masked hearing thresholds. Animals were tested at 26 frequencies over a range from $200 \mathrm{~Hz}$ to $100 \mathrm{kHz}$ using pure tones. The test tone amplitudes covered a range of $20 \mathrm{~dB}$ re 1 microPascal including the hearing threshold of the animal at that frequency. Hearing thresholds varied from $87.5 \mathrm{~dB}$ to $125.5 \mathrm{~dB}$ depending on frequency, masking noise intensity and individual animal. Data was analyzed to determine characteristic relationships between response time and amplitude of test tone for each frequency and animal. The two whales responded 
significantly slower $(640 \mathrm{~ms}, 0.001)$ than the four dolphins $(430 \mathrm{~ms})$. As in terrestrial animals, reaction time became shorter as stimulus strength increased. At threshold, median response time across frequencies within each animal varied about $150 \mathrm{~ms}$.

\section{$1: 20$}

2pAB2. What can be learned from one of nature's most advanced biosonars: Discussion on Bottlenose dolphins echolocation waveforms with respect to echolocation tasks in shallow water. Marc P. Olivieri (Broadband Sensor Div., GORCA Technologies, Inc., 300 W. Rte. 38, Moorestown, NJ 08057, olivieri@gorca.com)

This paper presents recent findings on the biosonar echolocation waveforms of Bottlenose dolphins (Tursiops truncatus). The complex spectral shape of the waveforms (bimodal spectrum) and the dynamics of the click trains are discussed. It is shown that these waveforms have not only the smallest possible time-bandwidth product, but that they are in fact minimum phase waveforms (i.e., the phase is a function of the autospectrum). The characteristics of the waveforms including time/frequency distribution, autocorrelation, and the bimodal spectral structure of these waveforms are discussed. An interpretation of these features is proposed with respect to echolocation tasks in shallow water. It is shown how these waveforms may be used for optimizing both near range resolution and sidelobe levels in the processed echo waveforms. Examples are shown on how GORCA Technologies, Inc. (GTI) exploits such features in the development of a biomimetic SONAR. Finally, this paper presents examples of GTIs proprietary broadband adaptive algorithm performance, using animal echolocation data. In the data used for this study, the animal performed a three-alternative match-to-sample task (data courtesy of the biosonar program at SPAWAR San Diego). [Work sponsored by ONR.]

\section{$1: 35$}

2pAB3. Nocturnal location and activity of Pacific coast bottlenose dolphins determined by a sonobuoy array. Eric S. Howarth and R. H. Defran (Cetacean Behavior Lab., San Diego State Univ., 5500 Campanile Dr., San Diego, CA 92182, ehowarth@mail.sdsu.edu)

Pacific coast bottlenose dolphins (Tursiops truncatus) move offshore, presumably to feed as the sun sets [Hanson and Defran (1993), Ward (1998), Day (1998)] and are most often found inshore on the surfline at sunrise. The purpose of this research was to document their activity and location during nocturnal hours. A sonobuoy array was deployed in a triangle configuration centered approximately $500 \mathrm{~m}$ from the mean low low water line at Torrey Pines State Beach, CA. Surveys were conducted for 11 nights between October 1999 and November 1999 and for 21 nights between August 2000 and December 2000. Dolphin vocalizations were detected on 26 of 33 nights.

\section{1:50}

2pAB4. Specialization of the auditory processing in harbor porpoise, characterized by brain-stem potentials. Nikolay G. Bibikov (N. N. Andreyev Acoust. Inst., Shvernik St., 4, Moscow 117036, Russia, nbibikov@hotmail.com)

Brain-stem auditory evoked potentials (BAEPs) were recorded from the head surface of the three awaked harbor porpoises (Phocoena phocoena). Silver disk placed on the skin surface above the vertex bone was used as an active electrode. The experiments were performed at the Karadag biological station (the Crimea peninsula). Clicks and tone bursts were used as stimuli. The temporal and frequency selectivity of the auditory system was estimated using the methods of simultaneous and forward masking. An evident minimum of the BAEPs thresholds was observed in the range of $125-135 \mathrm{kHz}$, where the main spectral component of speciesspecific echolocation signal is located. In this frequency range the tonal forward masking demonstrated a strong frequency selectivity. Offresponse to such tone bursts was a typical observation. An evident BAEP could be recorded up to the frequencies $190-200 \mathrm{kHz}$, however, outside the acoustical fovea the frequency selectivity was rather poor. Temporal resolution was estimated by measuring BAER recovery functions for double clicks, double tone bursts, and double noise bursts. The half-time of BAERs recovery was in the range of $0.1-0.2 \mathrm{~ms}$. The data indicate that the porpoise auditory system is strongly adapted to detect ultrasonic closely spaced sounds like species-specific locating signals and echoes.

\section{2:05}

2pAB5. Whale contribution to long time series of low-frequency oceanic ambient sound. Rex K. Andrew, Bruce M. Howe, and James A. Mercer (Appl. Phys. Lab., 1013 NE 40th St., Seattle, WA 98040, randrew@apl.washington.edu)

It has long been known that baleen (mainly blue and fin) whale vocalizations are a component of oceanic ambient sound. Urick reports that the famous "20-cycle pulses" were observed even from the first Navy hydrophone installations in the early 1950's. As part of the Acoustic Thermometry Ocean Climate (ATOC) and the North Pacific Acoustic Laboratory (NPAL) programs, more than 6 years of nearly continuous ambient sound data have been collected from Sound Surveillance System (SOSUS) sites in the northeast Pacific. These records now show that the average level of the ambient sound has risen by as much as $10 \mathrm{~dB}$ since the 1960's. Although much of this increase is probably attributable to manmade sources, the whale call component is still prominent. The data also show that the whale signal is clearly seasonal: in coherent averages of year-long records, the whale call signal is the only feature that stands out, making strong and repeatable patterns as the whale population migrates past the hydrophone systems. This prominent and sometimes dominant component of ambient sound has perhaps not been fully appreciated in current ambient noise models. [Work supported by ONR.]

\section{2:20}

2pAB6. Near-bottom hydrophone measurements of ambient noise and sperm whale vocalizations in the northern Gulf of Mexico. Joal Newcomb, Robert Fisher, Robert Field, Altan Turgut (Naval Res. Lab.-Stennis, Stennis Space Center, MS 39576 newcomb@nrlssc.navy.mil), George Ioup, Juliette Ioup (Univ. of New Orleans, New Orleans, LA 70148), Grayson Rayborn, Stan Kuczaj, Jerald Caruthers, Ralph Goodman (Univ. of Southern MS, Hattiesburg, MS 39402), and Natalia Sidorovskaia (Univ. of Louisiana at Lafayette, Lafayette, LA 70504)

Three bottom-moored hydrophones, $50 \mathrm{~m}$ above the bottom, were placed on a downslope line, ending at the largest concentration of sperm whale sightings in the northern Gulf of Mexico, in $600 \mathrm{~m}, 800 \mathrm{~m}$, and $1000 \mathrm{~m}$ water depths. These depths were chosen after upslope propagation modeling, using historical databases, showed transmission losses greater than $110 \mathrm{~dB}$ at hydrophones near the bottom in water shallower than 600 $\mathrm{m}$ for a $500 \mathrm{~m}$ deep source at the $1000 \mathrm{~m}$ contour. These autonomously recording hydrophones were environmental acoustic recording system (EARS) buoys obtained from the Naval Oceanographic Office. They were capable of recording signals up to $5500 \mathrm{~Hz}$ continuously for 36 days and were deployed from July 17 through August 21. During this period a major marine mammal exercise was being conducted at the surface by the Minerals Management Service and the National Marine Fisheries Service, with other government and university scientists, in which temporary acoustic recording devices were attached to the whales and the whales were monitored by a surface towed array. Our near-bottom measurements of ambient noise and sperm whale vocalizations are discussed and compared to those surface and on-whale measurements. [Research supported by ONR.] 


\title{
Session 2pAO
}

\section{Acoustical Oceanography: Acoustic Remote Sensing of the Ocean Environment}

\author{
David R. Palmer, Chair \\ Atlantic Oceanographic and Meteorological Laboratory, National Oceanographic and Atmospheric Administration, \\ 4301 Rickenbacker Causeway, Miami, Florida 33149
}

Contributed Papers

3:15

2pAO1. Estimating Europa's internal structure with ambient noise. Sunwoong Lee, Michele Zanolin, Aaron M. Thode, and Nicholas C. Makris (MIT, 77 Massachusetts Ave., Cambridge, MA 02139, makris@mit.edu)

Jupiter's moon, Europa, is believed to possess a vast liquid ocean beneath a thin ice surface, although the thickness of the ice shell and depth of the ocean still remain poorly constrained. The authors have previously discussed the possibility of using ambient noise to probe Europa's interior [Makris et al., Eos. Trans. Am. Geophys. Union 82(47), P22B-0552 (2001)]. Here the analysis is refined and extended. The probability density for high source level or "big bang" events from tidally induced surface cracks and meteor impacts is estimated. Such events are important because they are most likely to have returns from internal Europan strata that stand above the ambient noise level. Travel-time curves for various thicknesses are also presented and used to demonstrate that echo-sounding and tomographic techniques can be applied to estimate thickness of the ice layer, depth of the ocean, and internal temperature structure. Convective ice can also be detected by spectral analysis of surface waves, since significant contrast in shear wave speed is expected in the partial-melt-ice regime. Spectral analysis is also applied to analyze flexural modes of a thin ice shell, to estimate ice-shell thickness, and to potentially study the sediment layer on the seafloor.

\section{3:30}

2pAO2. Full field spatial correlation of range dependent surface generated noise in a stratified ocean with application to hurricane sensing. Joshua D. Wilson and Nicholas C. Makris (MIT, 77 Massachusetts Ave., Cambridge, MA 02139)

Much of the ambient noise in the ocean is generated by wind at the ocean surface. In the case of a hurricane, the wind speed, and therefore the source level, is not constant over the ocean surface. The wind speed in a hurricane can range from 0 knots at the eye to 140 knots at the eye wall where the eye and the eye wall are typically separated by only 10 kilometers. A spectral model for the spatial correlation of range dependent surface generated noise based on wave number integration is developed. This model accurately represents the acoustic field directly beneath the hurricane and at arbitrarily long ranges. It is used to illustrate the dramatic variation in the noise field caused by range dependent features like the eye and eye wall. Results of this model are shown for hurricanes in shallow and deep water environments, including Bay of Bengal and Mid-Atlantic regions. This model is also compared with a previous normal mode model [Perkins et al., J. Acoust. Soc. Am. 93, 739-752 (1993)]. This comparison shows that at long range the two models agree but at close range the wave number integration model is necessary to accurately represent the field.
$3: 45$

2pAO3. Accuracy limits on rapid assessment of gently varying bathymetry. B. Edward McDonald (U.S. Naval Res. Lab., Washington, DC 20375, mcdonald@sonar.nrl.navy.mil) and Charles Holland (Penn State Univ., State College, PA 16804)

Accuracy limits for rapidly probing shallow water bathymetry are investigated as a function of bottom slope and other relevant parameters. The probe scheme [B. E. McDonald and Charles Holland, J. Acoust. Soc. Am. 110, 2767 (2001)] uses a time reversed mirror (TRM) to ensonify a thin annulus on the ocean bottom at ranges of a few $\mathrm{km}$ from a vertical send/ receive array. The annulus is shifted in range by variable bathymetry (perturbation theory shows that the focal annulus experiences a radial shift proportional to the integrated bathymetry along a given azimuth). The range shift implies an azimuth-dependent time of maximum reverberation. Thus the reverberant return contains information that might be inverted to give bathymetric parameters. The parameter range over which the perturbation result is accurate is explored using the RAM code for propagation in arbitrarily range-dependent environments. [Work supported by NRL.]

\section{4:00}

2pA04. Revisiting Buldyrev's theory of the "axial wave" after 30 years. David R. Palmer (NOAA/Atlantic Oceanogr. and Meteorological Lab., 4301 Rickenbacker Cswy., Miami, FL 33149, david.r.palmer@noaa.gov), Natalie S. Grigorieva, and Gregory M. Fridman (St. Petersburg State Marine Tech. Univ., St. Petersburg, Russia)

We review and summarize some of the more interesting aspects of Buldyrev's theory of the axial wave as it relates to long-range propagation. Just as with the experimental observations, the theory describes the nearaxial acoustic signal in terms of early, geometrical-like arrivals followed by an incoherent arrival that cannot be described using geometrical acoustics. This incoherent arrival, or axial wave, results from a large number of waves which interfere with one another because of the presence of caustic structures along the waveguide axis. It is this interference which prevents the waves from being described in terms of geometrical acoustics. As the propagation range increases, the nature of the axial wave changes because the number of caustics increases and the region of interference associated with each one also increases. For a normal waveguide (axial energy arrives last) the axial wave "eats" a geometrical arrival as each new caustic is encountered along the propagation path. A range is ultimately reached beyond which no geometric arrivals are present in the signal. In underwater acoustics this maximum range is probably unphysically large. For an abnormal waveguide (axial energy arrives first), the axial wave "spits" out geometrical arrivals as the range increases.

\section{$4: 15$}

2pAO5. Investigation of near-axial interference effects in long-range acoustic propagation in the ocean. Natalie S. Grigorieva and Gregory M. Fridman (Dept. of Appl. Mathematics and Mathematics Modeling, St. Petersburg State Marine Tech. Univ., 3 Lotsmanskaya Str., St. Petersburg 190008, Russia, nsgrig@natalie.spb.su)

The observed time-of-arrival patterns from a number of long-range ocean acoustic propagation experiments show early geometrical-like arrivals followed by a crescendo of energy that propagates along the soundchannel axis and is not resolved into individual arrivals. The two- 
dimensional reference point source problem for the parabolic index of refraction squared is investigated to describe in a simple model case the interference of near-axial waves which resulted in forming the so-called axial wave and propose a formula for the axial wave in more general cases. Using the method proposed by Buldyrev [V. Buldyrev, Tr. Mat. Inst. Steklov 115, 78-102 (1971)], the integral representation for the exact solution is transformed in such a way to extract ray summands corresponding to rays radiated from the source at angles less than a certain angle, the axial wave, and a term corresponding to the sum of all the rays having launch angles greater than the indicated angle. Numerical results for the axial wave and the last term are obtained for parameters corresponding to long-range ocean acoustic propagation experiments. The generalization of the obtained formula for the axial wave to the case of an arbitrary range-independent sound speed is given and discussed. [Work supported by VSP Grant No. N00014-01-4003.]

TUESDAY AFTERNOON, 4 JUNE 2002

BRIGADE ROOM, 1:30 TO 3:45 P.M.

\title{
Session 2pEA
}

\section{Engineering Acoustics: Noise and Other Engineering Acoustics Topics}

\author{
Ahmet Selamet, Chair \\ Center for Automotive Research, The Ohio State University, 930 Kinnear Road, Columbus, Ohio 43212
}

Chair's Introduction-1:30

\section{Contributed Papers}

$1: 35$

2pEA1. The ambiguity of the acoustic source distributions for divergent source fields, with application to aerodynamic noise. Alan Powell (Dept. of Mech. Eng., Univ. of Houston, TX 77204-4006)

A given uniform applied line force of linear strength $F_{1}$ falls very rapidly to zero about the ends of its length $L_{1}$. The wave equation source term is monopolelike, $\partial F_{1} / \partial y_{1}$. The radiation is only from the opposite energy producing monopoles $\int\left(\partial F_{1} / \partial y_{1}\right) d y_{1}= \pm F_{1}$ at its extremities, forming a dipole $L_{1} F_{1}$. The divergence theorem transforms this into the exactly equivalent dipole distribution of strength $F_{1}$, total strength $F_{1} L_{1}$, now the "obvious" energy source for the given excitation. Analogously, a volume $L_{1} L_{2} L_{3}$ of a quadrupole distribution of uniform strength, say $\rho u_{1} u_{2}$ for an aerodynamic lateral quadrupole, also radiates like opposing line dipoles $\int\left(\partial \rho u_{1} u_{2} / \partial y_{2}\right) d y_{2} L_{3}= \pm \rho u_{1} u_{2} L_{3}$ along the $L_{1}$ sides $L_{2}$ apart (for lateral quadrupole $L_{2} \rho u_{1} u_{2} L_{1} L_{3}$ ), replaceable by monopoles $\int\left(\partial^{2} \rho u_{1} u_{2} / d y_{1} \partial y_{2}\right) d y_{1} d y_{2} L_{3}$ at the corners of the face $L_{1} L_{2}$ (where $\left.\partial^{2} \rho u_{1} u_{2} / \partial y_{1} \partial y_{2} \neq 0\right)$, yielding lateral quadrupole $L_{1} L_{2} \rho u_{1} u_{2} L_{3}$. With exactly the same fields, which can be considered physically realistic, like the force? The situation appears quite ambiguous; although note the fictitiousness of the monopoles, the appeal of $\partial \rho u_{1} u_{2} / \partial y_{2}$ appearing in momentum equation as an applied force, while the energy loss by all elements, directly, of the sound producing flow may perhaps be best associated with the quadrupole distribution.

\section{1:50}

2pEA2. The sound production characteristics of high-frequency impinging jet tones. Brenda S. Henderson (Mech. Eng. Dept., Kettering Univ., Flint, MI 48504-4898), James Bridges, and Mark P. Wernet (NASA Glenn Res. Ctr., Cleveland, OH 44135)

An experimental investigation into the production of high-frequency tones by a supersonic impinging jet using phase-locked shadowgraph photography and high-resolution digital particle image velocimetry (DPIV) is presented. High-frequency tones are often produced for nozzle-to-plate spacings less than two nozzle exit diameters and for nozzle pressure ratios (NPR) less than approximately 3.4, where NPR is equal to the stagnation pressure divided by the pressure at the nozzle lip. The high-frequency tones have lower amplitudes and less impulsive wavefronts than the more dominant low-frequency impinging tones and are presumably produced by a significantly different sound production mechanism. Periodic motion of the jet appears to be confined to the peripheral regions of the jet with no significant motion of the flow in the central regions of the jet behind the standoff shock wave. Unlike the jet motion associated with low-frequency impinging jet tones, recirculating regions are not formed and swept downstream behind the standoff shock wave and stationary stagnation regions are also not observed.

2:05

2pEA3. Shallow cavity flow tone experiments: Onset of locked-on states. D. Rockwell, J.-C. Lin, P. Oshkai, M. Reiss (Lehigh Univ., 19 Memorial Dr. W., Bethlehem, PA 18015-3085), and M. L. Pollack (Lockheed Martin Corp., Schenectady, NY 12301)

Fully turbulent inflow past a shallow cavity is investigated for the configuration of an axisymmetric cavity mounted in a pipe. Emphasis is on conditions giving rise to coherent oscillations, which can lead to locked-on states of flow tones in the pipe-cavity system. Evaluation of pressure gradients on plan views of three-dimensional representations allows extraction of the frequencies of the instability (Strouhal) modes of the cavity oscillation. These frequency components are correlated with traditional models originally formulated for cavities in a free stream. In addition, they are normalized using two length scales; inflow boundary-layer thickness and pipe diameter. These scales are consistent with those employed for the hydrodynamic instability of the separated shear layer, and are linked to the large-scale mode of the shear layer oscillation, which occurs at relatively long cavity length. The foregoing considerations provide evidence that pronounced flow tones can be generated from a fully turbulent inflow at very low Mach number. These tones can arise even for the extreme case of a cavity having a length over an order of magnitude longer than its depth. Suppression of tones is generally achieved if the cavity is sufficiently shallow.

\section{2:20}

2pEA4. Wind tunnel measurements of windscreen performance. Edward R. Maniet, Jr. (Textron Systems, 201 Lowell St., Rm. 3106, Wilmington, MA 01887, emaniet@systems.textron.com)

Microphone windscreens are routinely used in outdoor acoustic measurements to reduce wind noise pickup. Characterization of windscreen performance outdoors with real wind has several drawbacks notably that the test conditions cannot be controlled. Test methodology has been de- 
veloped that provides controlled, repeatable measurements of microphone windscreen performance in a laboratory setting. Wind noise measurements are performed using a high-speed/laminar-flow, low-noise wind tunnel that incorporates a large anechoic chamber. The wind tunnel is modified to produce a turbulent flow into which the microphone and windscreen under evaluation are placed. The turbulent velocity spectrum is measured using multi-axis hot-wire anemometers and compared to outdoor data to locate the best position in the turbulent flow to place the test article. Comparative performance measurements of several windscreen designs are presented.

\section{2:35-2:45 Break}

\section{2:45}

2pEA5. Acoustic characteristics of engine air cleaner boxes. Iljae Lee, Ahmet Selamet (Ctr. for Automotive Res., The Ohio State Univ., 930 Kinnear Rd., Columbus, OH 43212, selamet.1@osu.edu), and Sean Li (DaimlerChrysler Corp.)

Air cleaner boxes placed on the induction system of engines play a critical role in the attenuation of airborn sound. The acoustic performance of such configurations is investigated experimentally and computationally in the absence of mean flow. The transmission loss of a fabricated rectangular box (prototype) with various inlet and outlet locations and extensions is predicted by a three-dimensional boundary element method. The predictions are then compared with experimental data obtained from an impedance tube setup. The results demonstrate that: (1) the variations of locations and extension lengths of inlet and outlet affect the acoustic behavior of the prototype at higher frequencies, as expected; (2) the transmission loss characteristics at low frequencies may be estimated by a one-dimensional analysis; and (3) the boundary element method is effective throughout the entire frequency range of interest in predicting the acoustic performance of air cleaner boxes with asymmetric shape and/or inlet and outlet extensions.

\section{3:00}

2pEA6. A parametric study of location effects on the sidebands of Helmholtz resonators using boundary element analysis. Christopher E. Shaw, David J. Moenssen, and John D. Kostun (Visteon Corp., 15200 Commerce Dr., North Dearborn, MI 48120, cshaw2@visteon.com)

An important noise source used to tune automotive interior sound levels and quality is the sound which radiates from the open end of the engine induction system-induction noise. Understanding this source and its tuning can be vital to produce the customer-desired sound inside the vehicle. The most traditional method to attenuate (or tune) induction noise is through the addition of passive devices, the most common of which is the Helmholtz resonator. An unfortunate side effect to such a device is that narrow bands of frequencies higher and lower than the bandwidth of the resonator, called sidebands, tend to be amplified by the device. The subject of this study is to qualify the effect of changing resonator location on these sidebands. This is studied by determining the system frequency response to a white noise input by performing boundary element analysis on a simplified air induction system. The location of the device is varied, and the magnitude and frequency shift of the sidebands are studied. Experimental validation is also provided for select models.
3:15

2pEA7. An experimental/numerical study of nonlinear standing waves in resonantors. Joshua R. Finkbeiner, Xiaofan Li, Ganesh Raman (Illinois Inst. of Technol., 10 W. 32nd St., E1, Chicago, IL 60616), Christopher Daniels, and Bruce Steinetz (NASA Glenn Res. Ctr., 21000 Brookpark Rd., M.S. 23-3, Brook Park, OH 44135)

The generation of shock-free high-amplitude pressure waves in resonators promises to become a powerful and useful technology. Lawrenson et al. [J. Acoust. Soc. Am. Aug. (1998)] described the generation of shockfree high-amplitude pressure using large equipment and resonators to produce the reported effects. An attempt is made to generate shock-free highamplitude pressure waves using relatively small resonators excited by a small linear motor. Ambient air is used as the working fluid. A small cylindrical resonator is tested resulting in the lack of the expected shocked waveform while a larger model of the same shape exhibits shocking behavior. A small conical resonator produces shock-free pressure waves at resonance, but the amplitude of these waves is small. A larger cone resonator model produces shock-free pressure waves of higher amplitude. A large horn-cone resonator also produces shock-free high-amplitude pressure waves. A numerical model solves the governing equations using a shooting method. The numerical results are compared to the experimental results. The difficulties caused by structural resonances, which are separate from the desired fluid resonances and caused by approaching the natural frequency of the linear motor/resonator structure, are shown and discussed.

\section{$3: 30$}

2pEA8. Resonant acoustic measurement of vapor phase transport phenomenon in porous media. Richard Schuhmann (Dept. of Civil \& Environ. Eng., Penn State) and Steven Garrett (Grad. Prog. in Acoust., Penn State)

Diffusion of gases through porous media is commonly described using Fick's law and is characterized by a gas diffusion coefficient modified by a media-specific tortuosity parameter. A phase-locked-loop resonance frequency tracker [J. Acoust. Soc. Am. 108, 2520 (2000)] has been upgraded with an insulated copper resonator and a bellows-sealed piston instrumented with an accelerometer. Average system stability (temperature divided by frequency squared) is about $180 \mathrm{ppm}$. Glass-bead-filled cores of different lengths are fitted into an o-ring sealed opening at the top of the resonator. The rate at which the tracer gas is replaced by air within the resonator is controlled by the core's diffusion constant. Mean molecular weight of the gas mixture in the resonator is determined in real time from the ratio of the absolute temperature to the square of the fundamental acoustic resonance frequency. Molecular weight of the gas mixture is determined approximately six times per minute. Changes in the gas mixture concentration are exponential in time (within $0.1 \%$ ) over nearly two decades in concentration. We will report diffusion constants for two different sizes of glass beads, in samples of five different lengths, using two different tracer gases, to establish the validity of this approach. [Work supported by ONR.] 


\title{
Session 2pMUa
}

\section{Musical Acoustics: Free Reed Instruments: Historical Perspective and Recent Research}

\author{
James P. Cottingham, Chair \\ Physics Department, Coe College, Cedar Rapids, Iowa 52402
}

Chair's Introduction-1:00

Invited Papers

1:05

2pMUa1. From Kratzenstein to Wheatstone: Episodes in the early history of free reed acoustics. James P. Cottingham (Phys. Dept., Coe College, Cedar Rapids, IA 52402, jcotting@ coe.edu )

In 1780 C. G. Kratzenstein published a paper in St. Petersburg describing a machine which produced vowel sounds using free reeds with resonators of various shapes. This marks a convenient, if arbitrary, starting point for the history of the free reed musical instruments of European origin. These instruments developed rapidly, and by 1850 the accordion, concertina, harmonica, reed organ, and harmonium all had been invented and developed into more or less final form. A key figure in this period is Charles Wheatstone, who not only published papers on acoustical research but was also an inventor and commercially successful manufacturer of musical instruments, most notably the Wheatstone English concertina. Much of Wheatstone's research in acoustics and almost all of his work as an inventor of musical instruments involved free reeds. This paper presents some episodes in the development of the free reed instruments and some examples of acoustical research involving free reeds during the 18th and 19th centuries.

$1: 35$

2pMUa2. On Weber's theory of a free reed coupled to a resonator. Jonas Braasch (Institut für Kommunikationsakustik, Ruhr-Universität Bochum, 44780 Bochum, Germany)

In the 1820s Wilhelm Weber investigated the effect of resonator length on the frequency of a free reed coupled to a resonator. His aim was to build a device to generate a reference frequency since he was not satisfied with the accuracy of tuning forks. Weber's goal was to compensate the small dependence of frequency on blowing pressure and temperature by coupling a resonator to the free reed. Besides conducting a series of experiments Weber was the first to formulate a theory to estimate the frequency of a free-reed/resonator system. His theory is based on Euler's solution for two coupled pendulums with the free reed and the air column of the resonator as the two oscillators. Although some of his assumptions were not quite correct, Weber was able to predict some of the experimental results quite accurately by assuming that the oscillators are synchronized. However, because the free reed by itself was not frequency dependent on blowing pressure and temperature in his theory, he was not able to find a solution for compensating the frequency of the free reed using a resonator. In the presentation Weber's work will be introduced and compared to present theories.

2pMUa3. Measurements of reed vibration and pressure variation of the sho, the Japanese mouth organ. Takafumi Hikichi and Naotoshi Osaka (NTT Commun. Sci. Labs., NTT Corp., 3-1, Morinosato Wakamiya, Atsugi, Kanagawa 243-0198, Japan, hikichi@brl.ntt.co.jp)

Measurements of reed vibration and pressure vibration of the sho were carried out. Reed displacement, sound pressure at both sides of the reed, and radiated sound pressure at the open end of the pipe were measured using an experimental sho model made from an acrylic pipe and a metal reed. The kashira, or cavity, was also made from an acrylic box, where the pipe is mounted so that the reed vibration can be measured by a laser displacement sensor. The measurement results show that reeds vibrate as a sinusoidal, and that, in contrast with earlier results obtained by experiments on harmonium reeds [J. P. Cottingham, C. J. Lilly, and C. H. Reed, 137th meeting of the ASA and the 2nd Convention of the EAA, pp. 14-19 (1999)], the amplitude of reed vibration increases with increasing blowing/drawing pressure. The sound pressure inside the tube shows peaks when the reed reaches its maximum displacement, and the sound pressure oscillates twice when the reed oscillates once. Further, sound pressure inside the kashira, i.e., at the upstream of the reed, shows high frequency oscillation compared with reed vibration. This result implies that the shape and volume of the kashira may significantly affect the sound characteristics. 


\section{Contributed Paper}

2pMUa4. Does the European free reed really originate from Asia? Jonas Braasch (Institut für Kommunikationsakustik, Ruhr-Universität Bochum, 44780 Bochum, Germany) and Christian Ahrens (Ruhr-Universität Bochum, 44780 Bochum, Germany)

Free reeds can be found in European instruments like the accordion and reed organ. Christian Gottlob [Theophil] Kratzenstein was the first scientist in Europe to use a free reed when he built his speech-imitating machine, and it is believed throughout literature that he copied the mechanism of a free reed from the Asian mouth organ. However, after analyzing Kratzenstein's original work (Tentamen Resolvendi Problema ab Aka- demia Scientiarum Imperiali Petropolitana ad Annum 1780 Publicae Propositum) it was rather concluded that his free-reed design was modified from a striking reed pipe of an organ because he recorded that he wanted to reduce the rattle of his speech-imitating machine by modifying the reed. Furthermore, he did not mention the mouth organ at all in his work. An additional evidence for this hypothesis is his detailed figures of his speech imitating machine. They show clearly that the only difference between his free reed and the striking reed pipe of an organ is the smaller size of the reed in order to fit through the shallot. In contrast, Asian free reeds are flat, have no tuning wire, and the reed and reed opening are made from the same piece of metal.

\section{2:50-3:00 Break}

\section{Invited Papers}

\section{3:00}

2pMUa5. Laboratory measurements on free reeds from the reed organ, accordion, and khaen. Michael Busha (Phys. Dept., Grinnell College, Grinnell, IA 50112), James P. Cottingham, and Philip D. Koopman (Coe College, Cedar Rapids, IA 52402)

Several series of experimental measurements have been made on the motion of air-driven free reeds. These include individual reed organ reeds mounted on a laboratory wind chamber, accordion reeds mounted on the accordion reed block, and khaen reeds in individual bamboo pipes, in which the symmetric free reed is coupled to the pipe resonator. Measurements of reed displacement and velocity as a function of time have been made using a variable impedance transducer and a laser vibrometer system. Additional measurements made for each type of reed include sound pressure and air flow waveforms, as well as variation of sounding frequency and amplitude of vibration with blowing pressure. In comparing the sound pressure and air flow waveforms among the various types of reeds or for the same reed at different blowing pressures, differences can be understood in terms of the configuration of the reed and reed frame system and the amplitude of reed vibration. Some of the results can be interpreted in terms of simple theoretical models.

2pMUa6. From time domain simulation of diatonic harmonica, proposal of a minimal free reed model. Millot Laurent and Ambroise Daniel (Laboratoire d'Acoustique Musicale, 11 Rue de Lourmel, 75015 Paris, France)

Chromatical playing on diatonic harmonica can be explained and reproduced using a temporal model. This model features a nonlinear vocal track, two free reeds and interaction of these three elements. The agreement between numerical simulations and experiment will be illustrated. From these results, and considering the sinusoidal reed motion during normal playing, a minimal description of free reed will be proposed. The equivalent reed oscillator will be derived from comparisons between quasistatic and modal description of the reed displacement; it will be shown that the modal description is a good choice. Then, a sufficient description of the flow through each reed will be built. Waveforms and related sounds will be presented for each tested assumption and will illustrate the fact that this flow must rely on the local reed displacement. Application of this model will be discussed in the case of other free reed instruments.

\section{4:00}

2pMUa7. Acoustic coupling between oral tract and diatonic harmonica: Recent observations. James Antaki, Henry Bahnson, and Greg Burgreen (Dept. of Surgery and Bioengineering, Univ. of Pittsburgh, Pittsburgh, PA)

The diatonic harmonica is arguably one of the most "vocal" of all reed instruments. Due to the relatively short distance, thus tight coupling, between the player's oral cavity and the instrument, relatively subtle changes in the oral tract may effect dramatic modulations of both frequency and spectral content of the sound produced. Over the past several years we have endeavored to improve the understanding of this interaction, as well as the coupling between reeds of a given cell. This presentation will discuss our most recent observations. Benchtop experiments were conducted by laser vibrometer to measure the simultaneous motion of the reeds. Studies on human subjects were based on x-ray, ultrasonographic, and laryngoscopic imaging of players while sounding notes on the harmonica. Evidence of torsional instability has been observed, and analyzed by subsequent computational fluid dynamics simulation. 


\title{
Session 2pMUb
}

\section{Musical Acoustics: Free Reed Instruments: Concert Performance Featuring Henry Doktorski, Accordion and Howard Levy, Harmonica}

\author{
James P. Cottingham, Cochair \\ Physics Department, Coe College, Cedar Rapids, Iowa 52402 \\ James F. Antaki, Cochair \\ Department of Surgery and Bioengineering, University of Pittsburgh, Pittsburgh, Pennsylvania 15260
}

\begin{abstract}
Chair's Introduction-5:00
This performance technical session will feature classical accordionist Henry Doktorski and harmonica virtuoso Howard Levy.

Henry Doktorski is one of the leading concert accordionists in the United States and a recognized figure in international classical accordion circles. He has performed on accordion with the Pittsburgh Symphony Orchestra, the Tanglewood Festival Orchestra, the Cleveland Chamber Symphony, the McKeesport Symphony and the Pittsburgh New Music Ensemble. Henry Doktorski serves on the faculty of The City Music Center at Duquesne University as instructor of accordion and is the founder of The Classical Free-Reed, Inc., a nonprofit educational organization devoted to the accordion and classical music. (See the Classical Free Reed web site at: http://trfn.clpgh.org/free-reed/)

Howard Levy is a musician without limits. His musical adventures include journeys into jazz, pop, rock, world music, Latin, classical, folk, blues, country, theater, and film. He has appeared on hundreds of CDs, won a Grammy, won a Joseph Jefferson Award for Best Original Music for a Play, and performed many times on American and European television. Universally acknowledged as the world's most advanced diatonic harmonica player, Howard has developed a fully chromatic style on the standard 10-hole diatonic harmonica, revolutionizing harmonica playing and taking the instrument into totally new territory. (Visit his web site at: http://www.levyland.com/)
\end{abstract}

TUESDAY AFTERNOON, 4 JUNE 2002

STERLINGS ROOMS 2 AND 3, 1:00 TO 3:45 P.M.

\section{Session 2pPA}

\section{Physical Acoustics and Underwater Acoustics: Recent Developments in Wave Propagation in Random and Complex Media II}

\author{
D. Keith Wilson, Chair
}

AMSRL CI EE, U.S. Army Research Laboratory, 2800 Powder Mill Road, Adelphi, Maryland 20783-1197

Invited Papers

1:00

2pPA1. The effects of turbulent intermittency on scattering and estimates for the degree of saturation. David E. Norris (BBN Technologies, 1300 N. 17th St., Arlington, VA 22209, dnorris@bbn.com) and D. Keith Wilson (U.S. Army Res. Lab., Adelphi, MD 20783-1197)

\footnotetext{
Turbulent fluctuations in atmospheric wind and temperature fields are observed to be erratic in time; strong activity is typically interspersed with periods of relative calm. This property, referred to as turbulent intermittency, has an observable effect on the statistics of scattered acoustic signals. For fully saturated scattering, large intensity deviations about the mean result in a divergence from log-normal intensity probability density functions (pdfs) typically used to describe such statistics. Previous studies have developed the theory to predict the intensity pdf's that account for both turbulent intermittency and the degree of saturation [Norris et al., J. Acoust. Soc. Am. 109, 1871-1880 (2001); Wilson et al., J. Acoust. Soc. Am. 99, 3393-3400 (1996)]. The new formulation with intermittency is compared to the generalized gamma pdf previously proposed for propagation in random media. It is also compared to data collected over a $140 \mathrm{~m}$ line-of-sight path at 110 to $525 \mathrm{~Hz}$. Experimental characterization of the pdf parameters relating to intermittency and signal saturation is attempted with partial success. Strength/diffraction parameters and complex signal moments are computed to further define the scattering effects as a function of frequency and help identify limitations in the theory.
} 
2pPA2. Acoustic granular materials with pore size distribution close to log-normal. Kirill Horoshenkov and Mark Swift (Dept. of Civil and Environ. Eng., Univ. of Bradford, Bradford BD7 1DP, UK)

The water suction method is used to determine the parameters of the pore size distribution of a representative selection of loose and consolidated granular materials. It is shown that the experimentally determined pore size distribution in granular materials is often close to log-normal. The low- and high-frequency asymptotic behavior of the Biot viscosity correction function for media with log-normal pore size distribution is investigated and used to develop a simple rational approximation. This approximation is used to predict accurately the acoustic characteristic impedance and propagation constant for this class of materials. Unlike many available theoretical models for the acoustic properties of porous media which involve empirical shape factors, the proposed approximation is based entirely on four routinely measurable nonacoustic parameters: the porosity, flow resistivity, tortuosity, and the standard deviation of the pore size. The theoretical predictions for the acoustic surface impedance and absorption coefficient of loose and consolidated granulates are compared against the experimental results. A good agreement is obtained throughout the considered frequency range. [The authors are grateful to the Engineering and Physical Sciences Research Council (EPSRC) in the UK (Grant GR/L54905) for the support of this work. The authors would like to thank Dr. P. Leclaire for his help with the water suction experiments.]

\section{$1: 40$}

2pPA3. Voronoi polygons and self-consistent technique used to compute the airflow resistivity of randomly placed fibers in glass wool. Viggo Tarnow (Dept. of Mech. Eng., Tech. Univ. of Denmark, Bygning 358, DK-2800 Kgs. Lyngby, Denmark)

Sound in glass wool propagates mainly in the air between glass fibers. For sound waves considered here, the distance between fibers is much smaller than the wavelength. Therefore, the sound velocity and attenuation can be computed from an effective mass density and compressibility. For simple harmonic waves at low frequencies, the effective mass density is determined by the friction between air and fibers. The friction is described by the airflow resistivity, which depends on frequency, but for frequencies below 1000 $\mathrm{Hz}$ in glass wool with density $15-30 \mathrm{~kg} / \mathrm{m}^{3}$, the resistivity to airflow is constant, and equal to the constant current value. A computation of resistivity from fiber density and diameter will be presented for a model of glass wool that consists of parallel randomly placed fibers with equal diameters. The computation is based on Voronoi polygons, and the results will be compared with results from a self-consistent technique.

\section{2:00-2:15 Break}

\section{Contributed Papers}

2:15

2pPA4. Scattering of acoustic evanescent waves by circular cylinders: Partial wave series solution. Philip L. Marston (Dept. of Phys., Washington State Univ., Pullman, WA 99164-2814)

Evanescent acoustical waves occur in a variety of situations such as when sound is incident on a fluid interface beyond the critical angle and when flexural waves on a plate are subsonic with respect to the surrounding fluid. The scattering by circular cylinders at normal incidence was calculated to give insight into the consequences on the scattering of the evanescence of the incident wave. To analyze the scattering, it is necessary to express the incident wave using a modified expansion involving cylindrical functions. For plane evanescent waves, the expansion becomes a double summation with products of modified and ordinary Bessel functions. The resulting modified series is found for the scattering by a fluid cylinder in an unbounded medium. The perfectly soft and rigid cases are also examined. Unlike the case of an ordinary incident wave, the counterpropagating partial waves of the same angular order have unequal magnitudes when the incident wave is evanescent. This is a consequence of the exponential dependence of the incident wave amplitude on the transverse coordinate. The associated exponential dependence of the scattering on the location of a scatterer was previously demonstrated [T. J. Matula and P. L. Marston, J. Acoust. Soc. Am. 93, 1192-1195 (1993)].

\section{2:30}

2pPA5. A new numerical method for wave propagation through assemblies of cylinders and spheres. Takeru Yano (Dept. of Mech. Sci., Hokkaido Univ., Sapporo 060-8628, Japan) and Andrea Prosperetti (Johns Hopkins Univ., Baltimore, MD 21218)

PHYSALIS is a new method for the numerical solution of a variety of problems (potential theory, Navier-Stokes equations, and others) involving cylindrical or spherical internal boundaries [A. Prosperetti and H. N. Oguz, J. Comput. Phys. 167, 196-216 (2001)]. At the heart of the method is the use of an exact analytical solution to transfer the boundary conditions from the surface of the inclusions to the neighboring grid nodes. This step avoids the difficulty deriving from the complex geometrical relationship between the internal boundaries and the underlying regular grid, with the added benefit that fast solvers can be used. In this work the method is adapted to two-dimensional acoustic scattering by cylinders as governed by the Helmholtz equation. As in prior applications, the method reveals itself highly efficient and of a relatively simple implementation. These features are illustrated on several problems. In particular, it is shown that the computational time grows much less than linearly with the number of cylinders, which permits the simulation of complex multiple scattering problems without large computational resources. [Work supported by The Japan Ministry of Education, Culture, Sports, Science and Technology, and by ONR.]

\section{$2: 45$}

2pPA6. Acoustic wave propagation in atmospheric boundary layer above a rough terrain. Lanbo Liu and Mark L. Moran (U.S. Army Cold Regions Res. and Eng. Lab.)

In this paper we will present the latest development in the numerical simulation of acoustic wave propagation in the lowest atmospheric boundary layer above a rough terrain with a finite difference time domain (FDTD) algorithm. It is of great interest to investigate the effect of dynamic turbulent boundary layer meteorology coupled with surface terrain on acoustic wave propagation in frequency bands relevant to battlefield sensor systems. Turbulent mixing in the vicinity of the earth-air boundary layer is the key factor contributing to acoustic signal fluctuations such as upward refraction, scattering, high attenuation, and coherence degradations. Using first principles acoustic wave equation expressions for a moving fluid will allow us to couple acoustic models with time varying, 3-D large eddy simulation (LES) results. The specific meteorological parameters are vector wind velocity, temperature, pressure, and density as a function of space and time. Our acoustic simulation uses a coupled system of first-order temporal and spatial differential equations relating wind velocity, pressure, and density variations. This system of equations was evaluated with an explicitly time-stepping finite difference numerical ap- 
proach. The coupling of LES and acoustic FDTD simulation will allow us to directly identify spectral portions of turbulent boundary layer meteorology contributing to short- and long-term acoustic signal variations.

\section{3:00}

2pPA7. The acoustics of plasma-sprayed, thick-thermal-barrier coatings. John Harris (Ctr. QEFP, Northwestern Univ., Evanston, IL 60208-3020)

Plasma-sprayed, thick-thermal-barrier coatings are very porous, approximately $2 \mathrm{~mm}$ thick, coatings used to insulate metal engine parts from high-temperature combustion processes. They are grown such that their elastic constants and density increase with depth; at the surface the elastic constants take small values, especially the shear modulus which is very small, but rapidly increase to those typical of the metal substrate. A Thomson-Haskell matrix technique is used to find numerically the eigenmode(s) along each vertical line through the coating and substrate, at each station along the direction of propagation. Using the lowest eigenmode, calculated at each station, a JWKB approximation to propagation in a coating with variable thickness is calculated, and from this the changes in travel time caused by varying degress of spalling at the surface are worked out. Using the two or three lowest eigenmodes for a coating of uniform thickness, a coupled-mode calculation is used to determine to what degree an interfacial inclusion couples the lowest eigenmode into the next higher one. In both cases the goal is to calculate the changes caused by the defects so that their presence may be detected. [Work supported by NSF.]

\section{3:15}

2pPA8. Performance of acoustic sensor arrays operating in atmospheric turbulence: Geometric acoustics regime. Sandra L. Collier and D. Keith Wilson (U.S. Army Res. Lab., AMSRL-CI-EE, 2800 Powder Mill Rd., Adelphi, MD 20783-1197)

The performance of acoustic sensor arrays operating in atmospheric turbulence with fluctuations described by a von Kármán spectrum has been investigated for strong diffraction in the presence of strong or weak scattering [Collier and Wilson, ASA June 2001 Meeting, ASA Dec. 2001
Meeting, J. Acoust. Soc. Am. (in submission)]. Here the previous analysis is extended to the geometric acoustics regime, i.e., to the weak diffraction and weak scattering limit. A model for the probability likelihood function is developed and the coherence function calculated. The Cramer-Rao lower bounds of the wavefront angles of arrival are investigated for geometric acoustics and are shown to reduce to the expected expression for the angle-of-arrival variance in the limit of zero noise.

\section{$3: 30$}

2pPA9. An empirical model for the frequency spectrum of surface pressure fluctuations. Michael Goody (NSWC-CD, Code 7250, 9500 MacArthur Blvd., West Bethesda, MD 20817-5700, GoodyMC@nswccd.navy.mil)

An empirical model is presented for the surface pressure fluctuations beneath a two-dimensional, zero-pressure-gradient, turbulent boundary layer. The model is based on the form of Chase's model [J. Sound Vib. 70 (1), 29-67 (1980)] given by Howe (Acoustics of Fluid-Structure Interactions (Cambridge U.P., Cambridge, 1998), p. 208]. The model of Chase is adjusted to better agree with several well-respected experimental data sets that cover the momentum Reynolds number range, $1400<\operatorname{Re}_{\theta}<23400$. The new model requires knowledge a priori of the free-stream velocity, friction velocity, displacement thickness, and kinematic viscosity. The new model also incorporates the observed scaling behavior of the pressure spectrum. The size of the midfrequency overlap region of the model spectrum is Reynolds-number dependent. Additionally, the model spectrum beneath flows with different Reynolds numbers collapse to a single curve that decays as $\omega^{-5}$ at high frequencies when normalized using viscous scales. By collapsing at high frequencies when normalized on viscous scales, the model offers a high degree of confidence when extrapolated to flows with a higher Reynolds number $\left(\operatorname{Re}_{\theta}>23400\right)$. Examples are given using analytically calculated flows that extend to $\operatorname{Re}_{\theta}=2.45 \times 10^{6}$. Integration of the model equation recovers the measured $p^{\prime} / \tau_{W}$ to within $8 \%$.

TUESDAY AFTERNOON, 4 JUNE 2002

GRAND BALLROOM 4, 2:00 TO 3:45 P.M.

\title{
Session 2pPP
}

\section{Psychological and Physiological Acoustics: Hearing Aid Design: Psychophysics and Signal Processing II}

\author{
Dianne J. Van Tasell, Cochair \\ Starkey Laboratories, 6600 Washington Avenue, South, Eden Prairie, Minnesota 55344 \\ Brent W. Edwards, Cochair \\ Sound ID, 3430 West Bayshore Road, Palo Alto, California 94303
}

\section{Contributed Papers}

2:00

2pPP1. Fast dynamic range compression method for multichannel hearing aids. David V. Anderson (Georgia Inst. of Technol., Atlanta, GA 30332)

Hearing loss with recruitment results in a frequency-dependent reduced effective dynamic range of the ear. The advance of digital hearing aid capabilities has led to the proliferation of multiband, compression algorithms directed at the recruitment problem. However, results have been mixed at best. We propose an alternative design methodology to designing multiband compressive aids based on attack and release times. In particular, multiband compression hearing aids should be matched to certain characteristics of the ear with a memoryless gain based on the bandlimited envelope function of each band. Under the proposed design method each band has a different effective time-constant. 
that the algorithms impose on the speech. In the cases described above, this attenuation resembles the piecewise-linear input-output characteristic

2pPP2. A scheme for noise suppression and spectral enhancement of speech to alleviate speech reception problems from loss of frequency selectivity. Johannes Lyzenga, Joost M. Festen, and Tammo Houtgast (Dept. of ENT/Audiol., VU Medical Ctr., Amsterdam, The Netherlands)

Even after sufficient amplification, hearing-impaired listeners often experience problems in understanding speech under noisy conditions. This may be caused by suprathreshold deficits such as loss of compression and reduced frequency selectivity. In this project we investigate a scheme in which speech and noise are processed before presentation to try and alleviate intelligibility problems caused by reduced frequency selectivity. The scheme contains three strategies, one in which the peak-to-valley ratios of selected modulations in the speech spectrum are enlarged, a second in which the overall speech spectrum is modified, and a third in which noise is suppressed before the two enhancement steps. An overlap-and-add (OLA) algorithm is used in the implementation. The effect of the speech processing is evaluated by measuring speech-reception thresholds (SRT) for sentences in speech noise, estimating the signal-to-noise ratio at which listeners can correctly reproduce $50 \%$ of presented sentences. Hearingimpaired and normal-hearing listeners were used. To simulate the hearing impairment resulting from a loss of frequency selectivity, we spectrally smeared the stimuli presented to the normal-hearing listeners. We found that the preprocessing scheme achieved a modest improvement of nearly 2 $\mathrm{dB}$ in the SRT for normal-hearing listeners. Data for hearing-impaired listeners are presently being collected.

\section{$2: 30$}

2pPP3. Directional hearing aid using hybrid adaptive beamformer (HAB) and binaural ITE array. Scott T. Shaw, Andy J. LaRow, Gary L. Gibian (Planning Systems, Inc., 12030 Sunrise Valley Dr., Reston, VA 20191, ggibian@plansys.com), LaGuinn P. Sherlock (Univ. of Maryland School of Medicine, Baltimore, MD 21201), and Robert Schulein (Etymotic Res., Elk Grove Village, IL 60007)

A directional hearing aid algorithm called the Hybrid Adaptive Beamformer (HAB), developed for NIH/NIA, can be applied to many different microphone array configurations. In this project the HAB algorithm was applied to a new array employing in-the-ear microphones at each ear (HAB-ITE), to see if previous HAB performance could be achieved with a more cosmetically acceptable package. With diotic output, the average benefit in threshold SNR was $10.9 \mathrm{~dB}$ for three $\mathrm{HoH}$ and $11.7 \mathrm{~dB}$ for five normal-hearing subjects. These results are slightly better than previous results of equivalent tests with a 3-in. array. With an innovative binaural fitting, a small benefit beyond that provided by diotic adaptive beamforming was observed: $12.5 \mathrm{~dB}$ for $\mathrm{HoH}$ and $13.3 \mathrm{~dB}$ for normal-hearing subjects, a $1.6 \mathrm{~dB}$ improvement over the diotic presentation. Subjectively, the binaural fitting preserved binaural hearing abilities, giving the user a sense of space, and providing left-right localization. Thus the goal of creating an adaptive beamformer that simultaneously provides excellent noise reduction and binaural hearing was achieved. Further work remains before the HAB-ITE can be incorporated into a real product, optimizing binaural adaptive beamforming, and integrating the concept with other technologies to produce a viable product prototype. [Work supported by NIH/ NIDCD.]

\section{$2: 45$}

2pPP4. Recruitment-of-loudness effects of attenuative noise reduction algorithms. Nathaniel Whitmal III (ECE Dept., Worcester Polytechnic Inst., Worcester, MA 01002) and Azadeh Vosoughi (Cornell Univ., Ithaca, NY 14853)

Hearing-impaired listeners have greater difficulty understanding speech in noise than normal-hearing listeners do. As a result, hearing aid users are often challenged by the inability of their hearing aids to improve intelligibility in noise. Several investigators have addressed this problem by using well-known signal processing methods (e.g., spectral subtraction, Wiener filtering) to enhance noise-corrupted speech. Unfortunately, these methods have failed to provide significant improvements in intelligibility. One possible explanation is the level-dependent nature of the attenuation observed in certain recruitment-of-loudness simulators. The purpose of this study was to compare the intelligibility of processed speech with that expected for recruitment-of-loudness simulation. Trials of the CUNY Nonsense Syllable Test were conducted with 12 normal-hearing listeners, using syllables that were mixed with additive noise at SNRs of 6,12 , and 18 dB. Input-output characteristics for the signals were measured and used to determine the effective threshold shift imposed by the algorithms. Comparisons of measured intelligibility scores with articulation index-based intelligibility predictions indicate that the behavior of such noise reduction algorithms can be successfully modeled as a form of mild sensorineural hearing loss.

\section{3:00}

2pPP5. Optimization of speech in noise with three signal processing algorithms for normal-hearing and hearing-impaired subjects. Bas A. M. Franck, Wouter A. Dreschler (Academic Medical Ctr., Dept. of Clinical and Exp. Audiol., D2-223, P.O. Box 1100 DD, Amsterdam, The Netherlands), and Johannes Lyzenga (Free Univ. Medical Ctr., Amsterdam, The Netherlands)

In this study a three-dimensional Simplex procedure was applied to optimize speech in noise by a combination of signal processing algorithms for different acoustic conditions and hearing losses. The algorithms used to span the three dimensions are noise reduction, spectral tilting, and spectral enhancement, respectively. Additionally, we studied the algorithms for their main effects and interaction effects within the optimization process. The subjects were asked to evaluate two consecutive, differently processed sentences on listening comfort. Three different noise types and two signalto-noise ratios $(\mathrm{S} / \mathrm{N})$ were used. Three groups of subjects participated: normal hearing, normal hearing with simulated impaired auditory filtering (by spectral smearing), and sensorineurally hearing-impaired subjects. For the normal-hearing group we applied $\mathrm{S} / \mathrm{N}=0 \mathrm{~dB}$. For the hearing-impaired and the simulated hearing-impaired subjects we applied $\mathrm{S} / \mathrm{N}=5 \mathrm{~dB}$. We will discuss the similarities and differences in the response patterns of the three groups. Also, the individual preferences will be related to the hearing capacity, and to the type of interfering noise. Finally, we will discuss differences in the perceptual features that are used to judge listening comfort of the fragments by normal-hearing and hearing-impaired subjects.

\section{3:15}

2pPP6. Physical modeling of the feedback path in hearing aids with application to adaptive feedback cancellation. Joanna L Hayes and Boaz Rafaely (Inst. of Sound and Vib. Res., Univ. of Southampton, Southampton SO17 1BJ, UK)

Hearing aid system modeling based on two-port network theory has been used previously to study the forward gain and the feedback path in hearing aids. The two-port modeling approach is employed in this work to develop an analytic model of the feedback path by reducing the model matrices to simplified analytic expressions. Such an analytic model can simulate the frequency response of the feedback path given the values of relatively few physical parameters such as vent dimensions. The model was extended to include variability in the feedback path due to slit leaks, for example. The analytic model was then incorporated in an adaptive feedback cancellation system, where the physical parameters of the model were adapted to match the actual feedback path and cancel the feedback signal. In the initial stage of this study, the ability of the model to match the frequency response of various measured feedback paths was studied using numerical optimization. Then, an adaptive filtering configuration based on the physical model was developed and studied using computer simulations. Results show that this new approach to adaptive feedback cancellation has the potential to improve both adaptation speed and performance robustness. 
which contains several biquad filters, when used with special software, automatically detects and suppresses peaks. The filters then further flatten and extend the hearing aid frequency response to $16 \mathrm{kHz}$, while the ap-

2pPP7. A novel method of improving sound quality and reducing acoustic feedback in hearing aids. Mead Killion, John French, Steve Viranyi, and David Preves (Etymotic Res., 61 Martin Ln., Elk Grove Village, IL 60007)

Most current hearing aids have relatively narrow bandwidths, when compared to high-fidelity equipment, and exhibit undamped peaks because the peaks are considered less troublesome than the problem of waxclogged dampers. Attempting to make hearing aids wider band has typically resulted in increased acoustic feedback problems. The recent availability of an off-the-shelf digital hearing aid integrated circuit amplifier, propriate CORFIG correction is added to the frequency response, producing a transparent sound. Open ear versus aided KEMAR recordings were produced using a live jazz trio and a string quartet. The sound quality ratings for eight commercially available digital hearing aids were obtained from several different listening panels. The new response equalization proved advantageous in all cases. The effects of eliminating the peaks in the response on maximum real ear gain achievable before onset of acoustic feedback oscillation will be reported.

TUESDAY AFTERNOON, 4 JUNE 2002

KINGS GARDEN NORTH, 1:00 TO 5:00 P.M.

\title{
Session 2pSA
}

\section{Structural Acoustics and Vibration: Pyroshock II}

\author{
Harry Himelblau, Chair \\ Space \& Communication Division, The Boeing Company, Rocketdyne Propulsion and Power, \\ Canoga Park, California 91309-7922
}

1:00

2pSA1. Statistical analysis of pyroshock data. William O. Hughes (NASA Glenn Res. Ctr., M/S 86-10, Cleveland, OH 44135-3191, william.o.hughes@grc.nasa.gov)

The sample size of aerospace pyroshock test data is typically small. This often forces the engineer to make assumptions on its population distribution and to use conservative margins or methodologies in determining shock specifications. For example, the maximum expected environment is often derived by adding 3-6 dB to the maximum envelope of a limited amount of shock data. The recent availability of a large amount of pyroshock test data has allowed a rare statistical analysis to be performed. Findings and procedures from this analysis will be explained, including information on population distributions, procedures to properly combine families of test data, and methods of deriving appropriate shock specifications for a multipoint shock source.

1:30

2pSA2. Pyroshock testing—shock simulation facilities. Vesta I. Bateman (Sandia Natl. Labs., P.O. Box 5800, Albuquerque, NM 87185-0555, vibatem@sandia.gov)

A variety of shock simulation facilities are available to simulate pyroshock events. These facilities range from bounded impact shock machines and electrodynamic shakers to resonant fixture techniques. This presentation will focus on the use of general purpose and tuned resonant fixture techniques including a unique tunable beam apparatus developed at SNL. Examples of application of the resonant fixture technique for both component and full-scale structure pyroshock simulations will be presented. Advantages and disadvantages of each technique will be discussed along with the usable frequency content and bandwidth.

2:00

2pSA3. Pyroshock testing—electrodynamic shakers. David O. Smallwood (Sandia National Labs., P.O. Box 5800, Albuquerque, NM 87185-0553, dosmall@sandia.gov)

Far field pyroshock (accelerations less than a few hundred grams, and bandwidths less than a few $\mathrm{kHz}$ ) can be simulated on electrodynamic shakers. Typically, the specification is in terms of the shock response spectrum (SRS). Wave forms are synthesized which will match the required SRS. The process is not unique, as many wave forms can have essentially the same SRS. Sometimes additional restrictions are placed on the synthesized wave form. Most common are restrictions on the duration of the wave form. The process of synthesizing wave forms, which will match an SRS and conform to the limitations of electrodynamic shakers, will be described. The methods used to reproduce these wave forms on the shaker will then be discussed. 
2pSA4. Pyroshock testing: Effects of equipment simulators. William O. Hughes (NASA Glenn Res. Ctr., M/S 86-10, Cleveland, OH 44135-3191, william.o.hughes@grc.nasa.gov)

It is critical to a spacecraft's mission success that flight equipment be properly qualified to its expected shock environment. Simulators of varying fidelity are often used to measure the shock levels during spacecraft shock tests. Pyroshock test data from a recent spacecraft separation test will be shown that illustrates how shock response spectra differ for avionics equipment simulators of both low and high fidelity. The effect of the simulator weight on the shock response will also be shown. Additionally, data showing the attenuation of pyroshock levels, including its reduction with distance and across joints will be discussed. This observed attenuation data generally supports the standard methodologies for predictions of shock attenuation.

$3: 15$

2pSA5. Pyroshock design. Allan G. Piersol (Piersol Eng. Co., 23021 Brenford St., Woodland Hills, CA 91364-4830, apiersol@pacbell.net)

The structural response velocities produced by pyroshocks are usually too low to cause structural damage beyond the immediate region of the pyrotechnic device where damage is intended. On the other hand, pyroshocks can cause malfunctions of equipment mounted near the pyrotechnic device, particularly equipment containing microelectronic or ceramic elements. These malfunctions are usually the result of the high frequency content (above $2 \mathrm{kHz}$ ) of pyroshocks, and are difficult to design against using conventional analysis procedures. On the other hand, adherence to simple design guidelines, which are summarized, can substantially suppress pyroshock-induced failures of equipment.

3:45-5:00

Panel Discussion

TUESDAY AFTERNOON, 4 JUNE 2002

GRAND BALLROOM 2, 1:00 TO 5:00 P.M.

\title{
Session 2pSC
}

\section{Speech Communication: Models of Phonetic Category Formation and Structure}

\author{
Lori L. Holt, Cochair \\ Department of Psychology, Carnegie Mellon University, 5000 Forbes Avenue, Pittsburgh, Pennsylvania 15213 \\ Andrew J. Lotto, Cochair \\ Department of Psychology, Washington State University, P.O. Box 644820, Pullman, Washington 99164-4820
}

Chair's Introduction-1:00

Invited Papers

1:05

2pSC1. Speech perception as complex auditory categorization. Lori L. Holt (Dept. of Psych. and Ctr. for the Neural Basis of Cognition, Carnegie Mellon Univ., 5000 Forbes Ave., Pittsburgh, PA 15213, 1holt@andrew.cmu.edu)

Despite a long and rich history of categorization research in cognitive psychology, very little work has addressed the issue of complex auditory category formation. This is especially unfortunate because the general underlying cognitive and perceptual mechanisms that guide auditory category formation are of great importance to understanding speech perception. I will discuss a new methodological approach to examining complex auditory category formation that specifically addresses issues relevant to speech perception. This approach utilizes novel nonspeech sound stimuli to gain full experimental control over listeners' history of experience. As such, the course of learning is readily measurable. Results from this methodology indicate that the structure and formation of auditory categories are a function of the statistical input distributions of sound that listeners hear, aspects of the operating characteristics of the auditory system, and characteristics of the perceptual categorization system. These results have important implications for phonetic acquisition and speech perception. 
2pSC2. Neuroimaging studies of phonetic category learning. Julie Fiez (Dept. of Psych. and Ctr. for the Neural Basis of Cognition, Univ. of Pittsburgh, 605 LRDC, Pittsburgh, PA 15260, fiez+@ @itt.edu)

The Japanese perception of /r/ vs /// is being used as a model system to explore a Hebbian-based model of adaptive plasticity. Prior behavioral work has demonstrated the effectiveness of an adaptive training regime and the utility of response feedback on the acquisition of the ability to discriminate a minimal speech pair (e.g., "rock" vs "lock"). Imaging, using fMRI methods, is now being used to explore the neural substrates that underlie changes in categorical perception. Two questions are being addressed: (1) in which brain areas can a differential response to /r/ vs /l/ be identified, and is activity in these regions influenced by training? and (2) can the effectiveness of feedback as a part of the training regime be explained by the recruitment of reward-related brain regions that support learning?

\section{1:55}

2pSC3. Effects of category learning on auditory perception and cortical maps. Frank H. Guenther (Dept. of Cognit. and Neural Systems, Boston Univ., 677 Beacon St., Boston, MA 02215, guenther@cns.bu.edu)

Our ability to discriminate sounds is not uniform throughout acoustic space. One example of auditory space warping, termed the perceptual magnet effect by Kuhl and colleagues, appears to arise from exposure to the phonemes of an infant's native language. We have developed a neural model that accounts for the magnet effect in terms of neural map dynamics in auditory cortex. This model predicts that it should be possible to induce a magnet effect for non-speech stimuli. This prediction was verified by a psychophysical experiment in which subjects underwent categorization training involving non-speech auditory stimuli that were not categorical prior to training. The model further predicts that the magnet effect arises because prototypical vowels have a smaller cortical representation than non-prototypical vowels. This prediction was supported by an fMRI experiment involving prototypical and non-prototypical examples of the vowel /i/. Finally, the model predicts that categorization training with non-speech stimuli should lead to a decreased cortical representation for stimuli near the center of the category. This prediction was supported by an fMRI experiment involving categorization training with non-speech auditory stimuli. These results provide strong support for the model's account of the effects of category learning on auditory perception and auditory cortical maps.

2pSC4. On the flexibility of phonetic categories. Alexander L. Francis (Speech and Hearing Sci., Univ. of Hong Kong, Prince Philip Dental Hospital 5F, 34 Hospital Rd) and Howard C. Nusbaum (Univ. of Chicago, Chicago, IL)

Research on phonetic category acquisition suggests that over the course of development children shift the relative weight given to various acoustic cues to particular phonetic categories [S. Nittrouer, J. Phon. 20, 1-32 (1992)]. Adults also show flexibility in acquiring second language categories and learning to recognize synthetic speech. In both cases listeners must shift attention among some acoustic cues and change the weighting and mapping of others. Recent research demonstrated that category-level feedback can induce adult listeners to shift attention between conflicting cues to native stop-consonant categories. Subsequent research showed that adult listeners also reorganize the distribution of their attention to acoustic cues in learning to better understand synthetic speech. Moreover, research on learning foreign phonetic contrasts suggests that listeners can learn to direct attention to unfamiliar acoustic cues. These results suggest that phonetic categories are flexible in terms of the nature and quality of the acoustic cues that define them. We argue that this flexibility is a consequence of the basic nature of the general mechanism that mediates speech perception: In order to resolve the problem of context-conditioned variability, listeners must dynamically modify attention to the acoustic signal in a context-dependent manner.

\section{2:45-3:00 Break}

2pSC5. Perceptual interference effects on phonetic categorization by second-language learners and cochlear implant patients. Paul Iverson (Dept. of Phonet. and Linguist., Univ. College London, 4 Stephenson Way, London NW1 2HE, UK)

This talk will outline a new theory that describes how distortions in auditory processing, due to language experience or to hearing impairment, can interfere with phonetic categorization processes. Experimental data will be presented on the perception of American English /r/ and /l/ by American and Japanese listeners. Native-language tests of adult cochlear implant patients, for the /t/ $/$ d/ contrast, will also be presented. The results suggest that the formation and adaptability of phonetic categories are impaired when the auditory processing of speech stimuli increases the salience of within-category acoustic variation.

2pSC6. Internal structure of phonetic categories: Some characteristics and constraints. Joanne L. Miller (Dept. of Psych., Northeastern Univ., Boston, MA 02115)

A widely held assumption in the speech perception literature for many years was that during the course of processing listeners derive an abstract phonetic representation and, in doing so, discard information about the fine-grained detail of the speech signal. However, more recent research has shown that the representations of speech are much richer than this emphasis on abstract categories would suggest, and that listeners retain in memory a substantial amount of fine-grained acoustic-phonetic information. One line of 
evidence for the richness of phonetic representations comes from research showing that phonetic categories are internally structured in a graded fashion, with some members of the category perceived as better exemplars (as more "prototypical") than others. In this talk I will describe findings from our research program that highlight some of the characteristics of these internally structured categories, and discuss how these characteristics place constraints on models of phonetic perception. [Work supported by NIDCD.]

3:50

2pSC7. Animal models of speech sound categorization. Andrew J. Lotto (Dept. of Psych., Washington State Univ., Pullman, WA 99164-4802, alotto@wsu.edu)

Several recent studies have examined phonetic category formation by training nonhuman animals (e.g., birds) to respond differentially to distributions of speech sounds. Animal models provide good tests of theories of perceptual category formation because one can completely control the input distributions and obtain a fine-grained sampling of responses to the stimulus space. In studies using vowel sound distributions, animals' response structures reveal two salient characteristics. First, there is a tendency to respond most strongly to stimuli that are furthest from the boundaries between training distributions. Second, there is evidence that the structure of responses is a function of the statistics of the training input. This can be seen as a peak in response for stimuli near the centroid of the input distribution. These same response structure characteristics can also be seen in data collected from humans in categorization tasks. It is possible that these two characteristics are indicative of two systems of learning: one functional and one statistical. A similar dichotomy has also been recently proposed in the visual categorization literature. The implications of these results for theoretical and computational models of phonetic acquisition will be discussed. [Work supported by NSF and NIH.]

\section{Contributed Papers}

\section{4:15}

2pSC8. The interaction of short-term and long-term memory in phonetic category formation. James D. Harnsberger (Dept. of Commun. Sci. \& Disord., Univ. of Florida, 336 Dauer Hall, P.O. Box 11742, Gainesville, FL 32611-7420)

This study examined the role that short-term memory capacity plays in the relationship between novel stimuli (e.g., non-native speech sounds, native nonsense words) and phonetic categories in long-term memory. Thirty native speakers of American English were administered five tests: categorial AXB discrimination using nasal consonants from Malayalam; categorial identification, also using Malayalam nasals, which measured the influence of phonetic categories in long-term memory; digit span; nonword span, a short-term memory measure mediated by phonetic categories in long-term memory; and paired-associate word learning (word-word and word-nonword pairs). The results showed that almost all measures were significantly correlated with one another. The strongest predictor for the discrimination and word-nonword learning results was nonword $(r$ $=+0.62)$ and digit span $(r=+0.51)$, respectively. When the identification test results were partialed out, only nonword span significantly correlated with discrimination. The results show a strong influence of shortterm memory capacity on the encoding of phonetic detail within phonetic categories and suggest that long-term memory representations regulate the capacity of short-term memory to preserve information for subsequent encoding. The results of this study will also be discussed with regards to resolving the tension between episodic and abstract models of phonetic category structure.
4:30

2pSC9. Formation of categories for complex novel auditory stimuli. Daniel Mirman, Lori L. Holt, and James L. McClelland (Ctr. for the Neural Basis of Cognition and Psych. Dept., Carnegie Mellon Univ., 5000 Forbes Ave., Pittsburgh, PA 15213,dmirman@andrew.cmu.edu)

Categorization of complex sounds with multiple, imperfectly valid cues is fundamental to phonetic perception. To study the general perceptual and cognitive processes that support complex sound categories, a novel stimulus set was created that allows tight control of category structure and input distributions. Stimuli were created from 300-ms noise bursts by applying bandstop filters at varying center frequencies and manipulating rise/fall time of stimulus onset and offset. Stimuli were assigned to one of two categories and presented to participants in a category identification and an $\mathrm{AX}$ discrimination task. Feedback was provided during identification trials, but not during discrimination tasks. Participants quickly learned to apply the category labels with high accuracy. Identification reaction times followed a pattern typical of speech stimuli with an apex in reaction time at category boundary. These results are consistent with formation of new auditory categories. Preliminary results indicate that discrimination performance is not tightly coupled with development of sharp identification functions and response-time peaks at category boundaries. Implications for mechanisms of speech categorization and category formation will be discussed. [Work supported by $\mathrm{CNBC}, \mathrm{NIH}$, and NSF.] 
Session 2pSP

\title{
Signal Processing in Acoustics: Signal Processing Techniques
}

\author{
David C. Swanson, Chair \\ Applied Research Laboratory, Pennsylvania State University, State College, Pennsylvania 16804
}

Contributed Papers

$1: 15$

2pSP1. Forward-scattered acoustic intensity from prolate spheroids. Brian R. Rapids and Gerald C. Lauchle (Grad. Prog. in Acoust. and Appl. Res. Lab., Pennsylvania State Univ., P.O. Box 30, State College, PA 16804)

In bistatic scattering geometries, the detection of a forward-scattered signal is particularly difficult because the incident and scattered waves combine into a simultaneous mixture. The result is that the amplitude of the scattered wave becomes masked by that of the incident wave. During detection of the scattered signal, conventional space-time processing techniques regard the source signal as interference and attempt to suppress it. While vector sensors alone possess an inherent directivity due to their fundamental nature, intensity vector sensors coherently measure the acoustic pressure and particle velocity components (or related quantity such as acceleration, displacement, or pressure gradient). The coherent measurement of both acoustic field parameters may provide unconventional information regarding the presence of an object because of their known relationship. It is hypothesized that techniques based upon these coherent measurements will be able to process the total acoustic field rather than filter the scattered signal from the incident signal during detection. Theoretical and available experimental results will be presented to describe these hypothesized capabilities. [Work supported by ONR, Code 321SS under Grant No. N00014-01-1-0108, Dr. James F. McEachern, project monitor.]

\section{$1: 30$}

2pSP2. Further investigations into performance metrics for underwater communications. Scott L. Whitney, Geoffrey S. Edelson, Ned B. Thammakhoune, and Michael S. Richman (BAE Systems, MER15-2651, P.O. Box 868, Nashua, NH 03061-0868, scott.1.whitney@baesystems.com)

Determining the relationship between the performance of an underwater acoustic data communications system and the operating environmental conditions is a problem that continues to plague researchers. The complexity of the time-varying channel is difficult to measure and model. Therefore an approach that uses metrics measured from data collected at sea to characterize the channel is attractive. As expected, preliminary assessments on limited data have shown that performance depends not only on environmental conditions, but also on system implementation. By extracting a variety of metrics, a better understanding of the subset that discriminate between good and bad performance can be developed. Also, by analyzing the relationship between certain metrics and performance, system limitations can be identified for re-evaluation. For example, a surprising result of the initial assessment of performance using a multichannel decision feedback equalizer on real data showed that sparseness of multipath arrivals may be an arbiter of performance [M. S. Richman et al., J. Acoust. Soc. Am. 110, 2619 (2001)]. Therefore changes to the algorithm that allows for sparse arrivals may improve performance. In this paper, a larger number of metrics from greater quantities of real-data and system configurations are measured and evaluated against equalizer results.

\section{$1: 45$}

2pSP3. Reverberation noise modeling using extreme value theory. Brian La Cour and Robert Luter (Appl. Res. Labs., Univ. of Texas, P.O. Box 8029, Austin, TX 78713-8029, blacour@arlut.utexas.edu)

Normalized matched filter output forms the basis of target detection in active sonar. In a target-free environment, the central theorem, if valid, predicts that the statistics of the envelope follow a Rayleigh distribution, and, to first approximation, this is indeed observed. However, well-known departures from the Rayleigh model are found in the tail end of observed distributions. Traditional approaches to this problem have focused on constructing a simple, parameterized, non-Rayleigh distribution which more closely models observations. This paper suggests a novel alternative which focuses on a robust method of modeling only the tails of the distribution in favor of the less important body. Results from extreme-value theory are used to fit a generalized Pareto distribution (GPD) to the empirical cumulative distribution function, conditioned on a large threshold value. [A random variable $X$ has a GPD if $P(X \leqslant x)=1-(1+\gamma x / \sigma)^{-1 / \gamma}$ for $x \geqslant 0$, $\sigma>0$, and $\gamma$ real; $\gamma=0$ is the exponential distribution.] Estimates of $\gamma$ and $\sigma$ are discussed for a broad range of active sonar data, and the results are compared with fits to other popular non-Rayleigh models. The origins of non-Rayleighness are also considered, including finite-size effects, spatial and temporal correlations, and nonuniformity.

\section{2:00}

2pSP4. Performance of conventional and fluctuation-based signal detection applied to atmospheric acoustics in the presence of transients. Thomas Null, Chris Clark (Mil Tec, NCPA, Coliseum Dr., University, MS 38677), and R. A. Wagstaff (Univ. of Mississippi, University, MS 38677)

One problem encountered in atmospherics acoustics is the detection of steady signals in the presence of loud transient noise. The ability to discriminate against loud transients is an attractive feature of fluctuationbased beamforming. A fluctuation-based beamformer was developed and there was a need to evaluate its performance for acoustic environments that have frequently occurring transients. Synthetic noise fields, which included loud transient noise, were created. Subsequently, receiver operating characteristic (ROC) curves could be produced in a Monte Carlo fashion. In order to provide a benchmark, the results of a conventional beamformer were similarly tested via ROC curves. These ROC curves allowed for comparison of the two beamformers under specific signal and noise conditions. In this presentation, the effects of transients on the outputs of both the conventional beamformer and the fluctuation-based beamformer are discussed. Particular interest is focused on the amplitude distribution of the outputs.

\section{2:15}

2pSP5. Construction of high frame rate images with Fourier transform. Hu Peng and Jian-yu Lu (Ultrasound Lab., Dept. of Bioengineering, The Univ. of Toledo, Toledo, OH 43606, jilu@eng.utoledo.edu)

Traditionally, images are constructed with a delay-and-sum method that adjusts the phases of received signals (echoes) scattered from the same point in space so that they are summed in phase. Recently, the 
relationship between the delay-and-sum method and the Fourier transform is investigated [Jian-yu Lu, Anjun Liu, and Hu Peng, "High frame rate and delay-and-sum imaging methods," IEEE Trans. Ultrason. Ferroelectr. Freq. Control (submitted)]. In this study, a generic Fourier transform method is developed. Two-dimensional (2-D) or three-dimensional (3-D) high frame rate images can be constructed using the Fourier transform with a single transmission of an ultrasound pulse from an array as long as the transmission field of the array is known. To verify our theory, computer simulations have been performed with a linear array, a 2-D array, a convex curved array, and a spherical 2-D array. The simulation results are consistent with our theory. [Work supported in part by Grant 5RO1 HL60301 from NIH.]

\section{2:30-2:45 Break}

\section{$2: 45$}

2pSP6. Using cross-frequency cost functions for broadband source localization and environmental inversion. Ethan P. Honda (Appl. Res. Labs., Univ. of Texas, 10000 Burnet Rd., Austin, TX 78758)

Cost functions that are constructed by coherently summing (model-todata) correlations over hydrophone pairs and frequency have been used successfully for source localization [E. K. Westwood, J. Acoust. Soc. Am. 91, 2777-2789 (1992)] as well as source localization and environmental inversion [Neilsen, J. Acoust. Soc. Am. (to be published)]. Although the coherent sum is usually taken over the same frequency for both data and model, it is shown that summing over other regions of the $f_{\text {data }} \otimes f_{\text {model }}$ space is also useful and may facilitate more efficient source localization. It is shown that lines of constant $f_{\text {data }} / f_{\text {model }}$ correspond to different source bearings. Although looking along lines of constant $f_{\text {data }} / f_{\text {model }}$ can be used as a crude form of spatial filtering, a new non-plane-wave spatial filter is also constructed that helps localize sources in the presence of multiple interferers. The spatial filter employed uses the environmental model to construct its set of basis functions and is therefore theoretically capable of spatially filtering in full 3-D as opposed to just bearing, as is done in adaptive beamforming.

\section{3:00}

2pSP7. Wigner-Ville representations for acoustic source localization. Zoi-Heleni Michalopoulou (Dept. of Mathematical Sci., New Jersey Inst. of Technol., Newark, NJ 07102) and Leon Cohen (Dept. of Phys. and Astron., Hunter College, City Univ. of New York, 695 Park Ave., New York, NY 10021)

Signal dispersion in a waveguide can be linked to source and receiver location as well as physical properties of the propagation medium. Traditionally, the main methods used for source localization in the ocean and in geoacoustic inversion problems have been based on the use of the spectrogram for the extraction of the dispersion information. In this work we explore the possibility of using other time-frequency transforms because other transforms, such as the Wigner-Ville representation, reflect the dispersion properties of the waveguide more accurately than conventional spectrograms. We apply the Wigner-Ville distribution, in conjunction with sound propagation models, for inversion with underwater sound. Results with synthetic data calculated for simplified ocean media indicate the potential of the approach for successful parameter estimation. [Work supported by ONR.]

\section{3:15}

2pSP8. Multichannel active noise control and acoustic equalization using fast affine projection algorithms. Martin Bouchard (School of Information Technol. and Eng., Univ. of Ottawa, 161 Louis Pasteur, Ottawa, ON K1N 6N5, Canada)

In the field of adaptive signal processing, it is well known that affine projection algorithms or their low-computational implementations, fast affine projection algorithms, can produce a good trade-off between convergence speed and computational complexity. Although these algorithms typically do not provide the same convergence speed as recursive-leastsquares algorithms, they can provide a much improved convergence speed compared to stochastic gradient descent algorithms, without the high increase of the computational load or the instability often found in recursiveleast-squares algorithms. In this presentation, multichannel fast affine projection algorithms are introduced for active noise control or acoustic equalization. Multichannel fast affine projection algorithms have been previously published for acoustic echo cancellation, but the problem of active noise control or acoustic equalization is a very different one, leading to different structures. The computational complexity of the new proposed algorithm is evaluated, and it is shown through simulations that not only can the new algorithm provide the expected trade-off between convergence performance and computational complexity, it can also provide the best convergence performance (even over recursive-least-squares algorithms) when non-ideal noisy acoustic plant models are used in the adaptive systems.

\section{3:30}

2pSP9. A comparison of algorithms and the development of a new fast convergence and reduced computational load algorithm for multichannel active noise control. Martin Bouchard and Scott Norcross (School of Information Technol. and Eng., Univ. of Ottawa, ON K1N 6N5, Canada)

In this presentation, the three main factors that affect the convergence speed of learning algorithms for adaptive FIR filters used in multichannel active noise control are described. Based on these three factors, a comparison of several adaptive FIR filter algorithms for multichannel active noise control is done, including several existing algorithms and a few unpublished algorithms. Of the unpublished algorithms, one algorithm has the potential for optimal convergence speed, and this algorithm is described in more detail in the presentation. The algorithm combines the use of recursive-least-squares algorithms with the use of an inverse model of the multichannel acoustic plant between the actuators and the error sensors. The resulting algorithm is called the multichannel inverse delaycompensated filtered-x RLS algorithm for active noise control. This algorithm can not only provide fast convergence, but for multichannel systems it also provides a significant reduction of the computational load compared to the previously published algorithm with the fastest convergence speed. Simulation results are presented to validate the convergence behavior of the new proposed algorithm.

\section{$3: 45$}

2pSP10. Inverse source problem by convex optimization with constraints over the object space and signal field. Kenbu Teramoto (Dept. of Mech. Eng., Saga Univ., Saga-shi 8408502, Japan, tera@me.saga-u.ac.jp)

In the acoustical endscopy, due to the physical limitations, the transducer array is composed of a small number of elements and each interspacing is larger than the acoustical wavelength that is called a sparse array system. In such cases, avoiding the ill-posed problems, projection onto convex sets (POCS) methods are used with incorporating constraints about both the signal field and the object space. POCS, however, is based on the alternating projections paradigm, which has a slow-convergence property in general. Furthermore if inconsistency exists in the set of constraints, this POCS algorithm cannot guarantee the convergence to the optimal estimate. The proposed algorithm is based on convex optimization over the direct product of the object space and the observed signal field. By acoustical experiments, it is proved that the proposed algorithm has the following improvements: (1) Targets can be identified when unknown components exist in the transfer function. (2) Transient behavior of the convergence becomes more stable than that of POCS algorithm. (3) Instability caused by the inconsistency in the constraints can be reduced. (4) Artifacts caused by the spurious lobes can be reduced under the condition that the interspacing of transducer elements is larger than the wavelength. 


\title{
Session 2pUW
}

\section{Underwater Acoustics: Propagation in Sediments and Inverse Methods}

\author{
Kyle M. Becker, Chair \\ Applied Research Laboratory, Pennsylvania State University, P.O. Box 30, State College, Pennsylvania 16804-0030 \\ Chair's Introduction-1:00
}

\section{Contributed Papers}

1:05

2pUW1. A two-way coupled mode simulation of sound propagation in a shallow water wedge. T. W. Yudichak, D. P. Knobles, and R. A. Koch (Appl. Res. Labs., Univ. of Texas, Austin, TX 78713-8029)

Near a shoreline an acoustic waveguide can be modeled by a wedgeshaped geometry with a penetrable bottom. The propagation of sound in such a waveguide was studied in a tank experiment conducted by Tindle et al. [J. Acoust. Soc. Am. 81, 275-286 (1987); 81, 287-294 (1987)]. An analysis of the time series data measured in this experiment was carried out by Royal et al. (unpublished), in which time series were simulated using a broadband, one-way, coupled normal mode approach. This analysis is extended using a two-way propagation approach that solves the integral equation form of the coupled mode equation. Also, the modal continuum is treated more realistically through the employment of a leaky mode decomposition. In particular, the importance of backscattering and coupling to the continuum is gauged by comparing the experimental data with the results of the two approaches.

\section{1:20}

2pUW2. Extensions to the method of complex images. John A. Fawcett (Defence Res. Establishment Atlantic, Dartmouth, NS B2Y 3Z7, Canada)

In an earlier paper [J. A. Fawcett, J. Acoust. Soc. Am. 108, 2791-2795 (2000)] the application of the method of complex images to approximating the half-space acousto-elastic Green's function was described. In this paper, extensions to this theory are presented. It is shown that the method can be used for layered half-spaces. For a uniform half-space, analytic time domain expressions are derived for a propagating pulse. By using the complex images in a manner analogous to image theory for a perfect waveguide, the method can be used to approximate a waveguide's Green's function. The Green's function described by the complex image method is accurate for near-field to far-field ranges and is ideal for many propagation and scattering modeling applications.

\section{$1: 35$}

2pUW3. T-wave sources, slopes, rough bottoms and continuum. Robert I. Odom and Darin J. Soukup (Appl. Phys. Lab., Univ. of Washington, 1013 NE 40th St., Seattle, WA 98105, odom@apl.washington.edu)

Bathymetry plays a strong role in the excitation of T-waves by breaking strict mode orthogonality and permitting energy from higher order modes to couple to the lower order modes comprising the T-phase. Observationally (Dziak, 2001) earthquakes with a strong strike-slip component are more efficient at generating T-waves than normal fault mechanisms with the same moment magnitude. It is shown that fault type and orientation correlates strongly with T-wave excitation efficiency. For shallow sources, the discrete modes contribute to the majority of the seismic source field, which is then scattered into the acoustic modes by irregular bathymetry. However, the deeper the earthquake source, the more important the continuum component of the spectrum becomes for the excitation. Deterministic bathymetry and random roughness enter the modal scattering theory as separate terms, and allow the relative contributions from the slope conversion mechanism and bottom roughness to be directly compared. [Work supported by the National Ocean Partnership Program.]

\section{$1: 50$}

2pUW4. Electrokinetic transduction of slow waves in porous media. Gareth Block and Nicholas P. Chotiros (Appl. Res. Labs., P.O. Box 8029, Univ. of Texas, Austin, TX 78713-8029, gblock@arlut.utexas.edu)

One candidate for modeling wave propagation in ocean sediments is Biot theory. It predicts two kinds of compressional waves: a fast wave, described by in-phase motion of the pore fluid and porous solid structure, and a slow wave, described by out-of-phase motion. Slow wave motion is heavily damped and has been difficult to measure directly using acoustic transducers. An electrokinetic (EK) transduction technique for studying slow waves is being developed which takes into account the surface chemistry of the grains and pore fluid salinity. EK measurements are advantageous because they are linked directly to out-of-phase, relative motion between the pore fluid and grain structure. EK transmission occurs when an applied voltage drives ionic (and thus fluidic) currents in an electrolytesaturated material. Conversely, EK reception is possible when fluid electrolyte motion in this material creates a measurable streaming potential. Progress on the experimental apparatus and theoretical model for the EK transmission case will be reported. [Work supported by ONR, Ocean Acoustics.]

\section{2:05}

2pUW5. Seismo-acoustic inversion of shallow water data using genetic algorithms. Timothy $H$. Ruppel (Naval Res. Lab., Stennis Space Center, MS 39529, tim.ruppel@nrlssc.navy.mil)

Peter Gerstoft's seismo-acoustic inversion system uses a Genetic Algorithm to search the parameter space and to estimate the errors in the inversion results, and allows the selection of one of several forward models in the inversion process. The current paper tests this system using data from an experiment conducted off the coast of Panama City by Steve Stanic in the summer of 2001. In the experiment, a vertical array was placed in shallow (6.62 $\mathrm{m}$ depth) water and ensonified by a submerged source at distances ranging from $20 \mathrm{~m}$ to $90 \mathrm{~m}$. In addition, a number of environmental measurements were taken, making this a suitable data set for acoustic inversion. The SAGA system will be used with several forward models for a comparison. [Work supported by the ONR Program Element No. 62435N.] 
autoregressive spectral estimator was investigated for determining hori-

2pUW6. A generalized inversion method: Simultaneous source localization and environmental inversion. Tracianne B. Neilsen and David P. Knobles (Appl. Res. Labs., Univ. of Texas, Austin, P.O. Box 8029, Austin, TX 78713-8029, neilsen@arlut.utexas.edu)

The problem of localizing and tracking a source in the shallow ocean is often complicated by uncertainty in the environmental parameters. Likewise, the estimates of environmental parameters in the shallow ocean obtained by inversion methods can be degraded by incorrect information about the source location. To overcome both these common obstaclesenvironmental mismatch in matched field processing and incorrect source location in geoacoustic inversions - a generalized inversion scheme is developed that includes both source and environmental parameters as unknowns in the inversion. The new technique called systematic decoupling using rotated coordinates (SDRC) expands the original idea of rotated coordinates [M. D. Collins and L. Fishman, J. Acoust. Soc. Am. 98, 16371644 (1995)] by using multiple sets of coherent broadband rotated coordinates, each corresponding to a different set of bounds, to systematically decouple the unknowns in a series of simulated annealing inversions. The results of applying the SDRC inversion method to data from the Area Characterization Test II experiment performed on the New Jersey continental shelf are presented. [Work supported by ONR.]

\section{$2: 35$}

2pUW7. Evaluation of an autoregressive spectral estimation technique for determining horizontal wave-number content in shallow water. Kyle M. Becker ${ }^{\mathrm{a}}$ (MIT/WHOI Joint Prog. in Oceanogr. and Oceanogr. Eng., Woods Hole Oceanogr. Inst., Woods Hole, MA 02543) and George V. Frisk (Woods Hole Oceanogr. Inst., Woods Hole, MA 02543)

Modal-based techniques for geoacoustic inversion require estimates of discrete horizontal wave numbers corresponding to the propagating modes in a shallow-water waveguide. For range-dependent waveguides, local wave-number content can be extracted as a function of range using methods analogous to short-time Fourier transform techniques as applied to non-stationary time series data. A limiting factor in these techniques is the aperture length, $L$, required to resolve closely spaced horizontal wavenumber content. Using classical spectral estimation methods, the limit of resolution is proportional to $1 / L$. In order to increase the spatial resolution of local horizontal wave-number estimates, the use of a high-resolution zontal wave-number content. The performance of the estimator was evaluated numerically using both time-series and spatial data. Results are presented of estimator performance for various aperture lengths using signals with increasing levels of additive noise and signals with closely spaced wave-number components. Comparisons are made with results of classical methods as well as other high-resolution methods. The results show the AR method to be well suited for wave-number estimation in shallow-water waveguides. [Work supported by ONR.] ${ }^{\text {a) }}$ Presently with the Acoustics Group, The Pennsylvania State Univ., Appl. Res. Lab., P.O. Box 30, State College, PA 16804,kmb166@psu.edu.

\section{$2: 50$}

2pUW8. Sediment tomography in the East China Sea: Compressional wave speed and attenuation inversions from mode travel time dispersion and Airy phase measurements. ${ }^{\text {a) }}$ Colin J. Lazauski, Gopu R. Potty, James H. Miller, Chuen-Song Chen (Dept. of Ocean Eng., Univ. of Rhode Island, Narragansett, RI 02882, lazauski@oce.uri.edu), and Peter H. Dahl (Appl. Phys. Lab., Univ. of Washington, Seattle, WA 98105)

This paper discusses ongoing data analysis results from the acoustic bottom interaction experiment conducted in May-June 2001 in the East China Sea as part of the Asian Seas International Acoustics Experiment (ASIAEX-2001). Using time-frequency scalograms of broadband signals, the modal arrivals and group speed minimums (Airy Phase) of several modes are clearly observed. The structure of the Airy Phase signal forms the basis of this inversion technique. Utilizing the Airy Phase group speed minimums and corresponding pressure amplitudes of each observable mode, the sediment compressional wave speed and attenuation as a function of depth are derived. The group speed minimum for each mode provides additional information on the compressional wave speed in the modal sediment depth penetration interval. Inverted speeds and estimated modal penetration depths are then used to develop the sediment profile. The estimated resolution is dependent on the number and frequency span of the observable modes. This method has the advantage of utilizing only one hydrophone to obtain rapid estimates of sediment properties. Additionally, it also complements other inversion schemes that can benefit from accurate a priori environmental information by limiting the size of the search parameter space. Estimated sediment properties from several areas are presented with verification from coring results. [Work supported by ONR.] ${ }^{\text {a) }}$ For Acoustical Oceanography Best Student Paper Award. 